\title{
Nano-Domains in Lead-Free Piezoceramics: A Review
}

\author{
Xiang $\mathrm{Lv}^{1}$, Xi-xiang Zhang ${ }^{2}$, and Jiagang $\mathrm{Wu}^{1 *}$ \\ ${ }^{1}$ Department of Materials Science, Sichuan University, Chengdu, P. R. China 610065 \\ ${ }^{2}$ Division of Physical Science and Engineering, King Abdullah University of Science and Technology, \\ Thuwal, Saudi Arabia 239955 \\ *Correspondence e-mail: wujiagang0208@163.com and msewujg@scu.edu.cn
}

\begin{abstract}
Reducing or even prohibiting the usage of toxic lead in electronic devices has become one of the most cutting-edge topics in multi-discipline. The recently proposed phase boundary engineering endows lead-free piezoceramics with comparable performance to those of part lead-based ones. The enhanced performance hinges on the multi-phase coexistence and complex domain structure, particularly the occurrence of nano-domains and polar nanoregions (PNRs). Although nano-domains were largely studied in lead-based piezoceramics, understanding nano-domains and PNRs of lead-free piezoceramics is in infancy and needs a systematic summary and an in-depth analysis. Here we summarized nano-domains and PNRs of three representative lead-free piezoceramics (i.e., potassium sodium niobate, barium titanate, and sodium bismuth titanate), focusing on their effects on macro performance. Firstly, we introduced the foundation and observation tools of domains. Then, we summarized nano-domains varying with phase structure, electric field, and temperature, as well as their effects on performance including piezoelectricity, strain, temperature stability, aging, and fatigue.
\end{abstract} Finally, we gave out our perspectives on the future of nano-domains, concentrating on nano-domain engineering. Therefore, this review helps better understand the nano-domains and PNRs of lead-free piezoceramics and further develop high-performance lead-free piezoceramics. 
Keywords: Lead-free piezoceramics; Nano-domains; PNRs; Performance; Physical mechanisms

Broader context: Piezoceramics can interconvert mechanical energy with electrical energy and are widely utilized in kinds of electronic devices. Currently used piezoceramics are mainly lead-based ones because they have excellent electrical properties and adequate stability. But the toxic lead contaminates the environment and harms human health, which goes against sustainable development. Therefore, the renew of Restriction of Hazardous Substances Directive (RoHS), a law by the European Union, has urged a more rapid development of high-performance lead-free piezoceramics. In particular, a report published by BCC Research claimed that the global market for lead-free piezoceramics is as high as \$ 172 million in 2019 and should grow up to \$ 443 million by 2024 . Therefore, developing high-performance lead-free piezoceramics has become one of the most cutting-edge topics in multidiscipline, including functional materials, environmental sciences, and electronic devices. Even though the recently enhanced performance in lead-free piezoceramics, it is still inferior to that of lead-based counterparts, and its physical mechanisms need to be further understood. Ferroelectric domains bridging micro atom arrangement and macro performance are crucial for understanding the physical mechanisms, particularly the nano-domains. Therefore, this review summarizes the nano-domains of lead-free piezoceramics, which helps develop lead-free piezoceramics to replace lead-based ones. 


\section{Content}

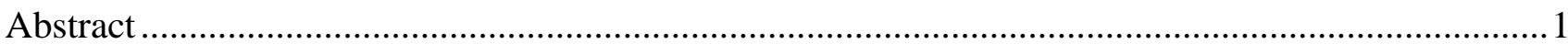

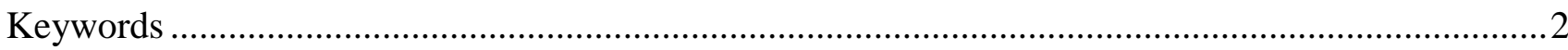

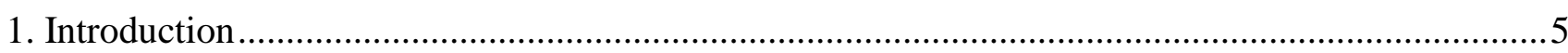

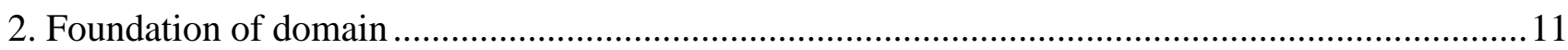

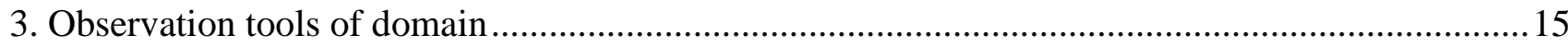

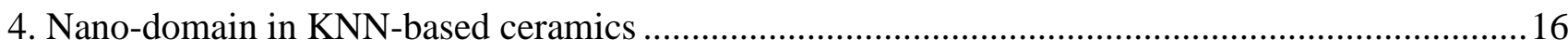

4.1 Nano-domain vs. Phase structure ............................................................................... 17

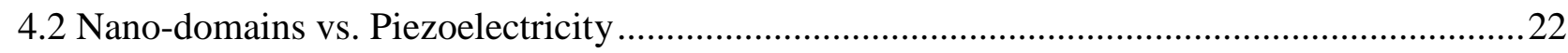

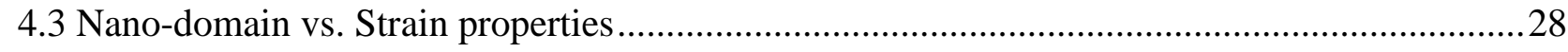

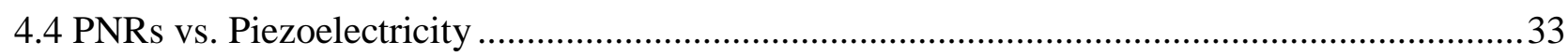

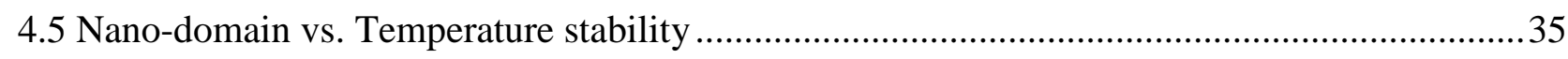

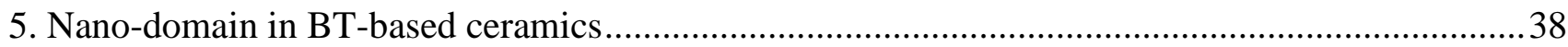

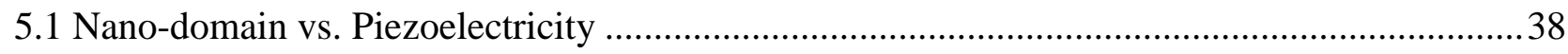

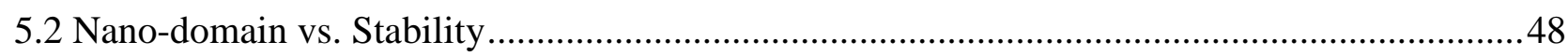

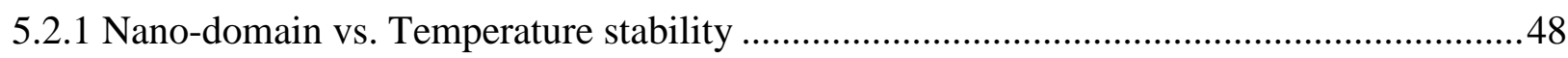

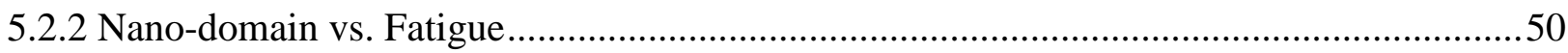

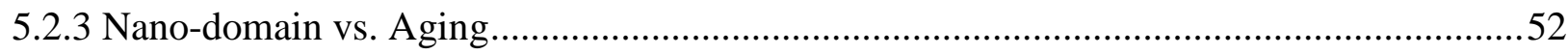

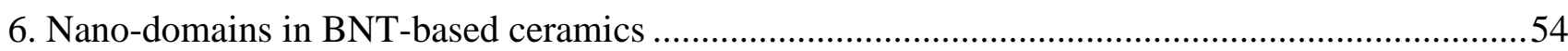

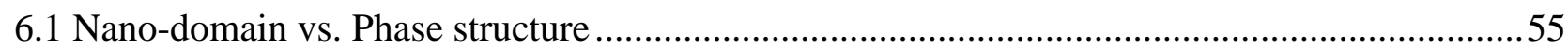

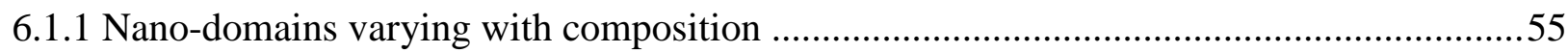

6.1.2 Nano-domains varying with temperature ..............................................................58

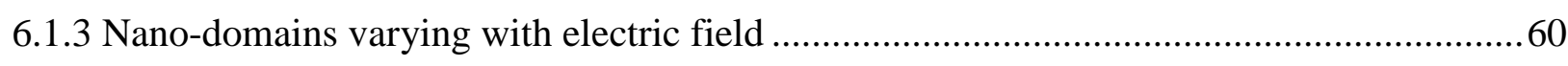




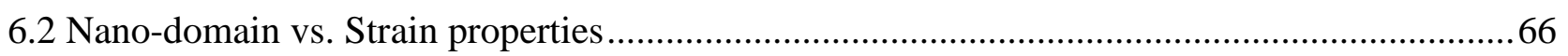

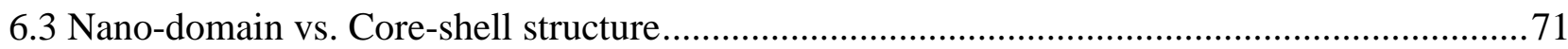

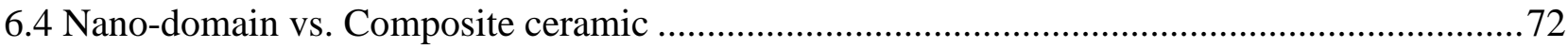

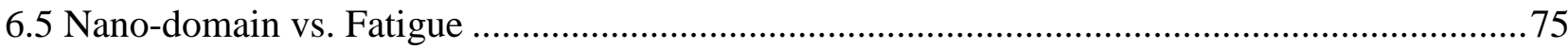

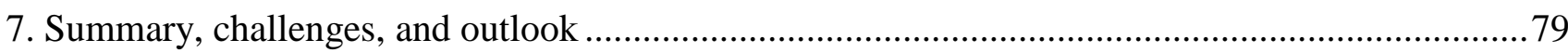

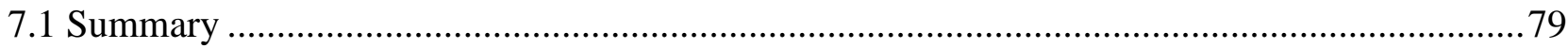

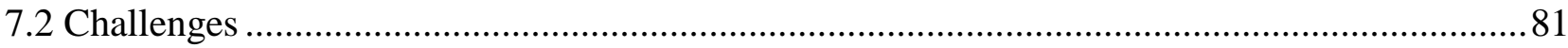

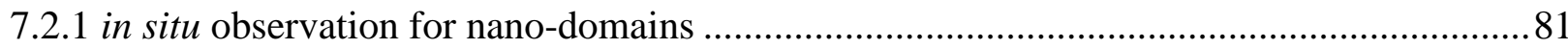

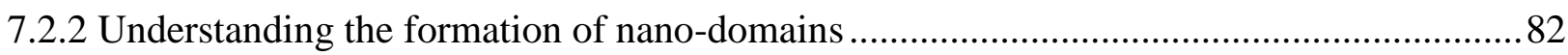

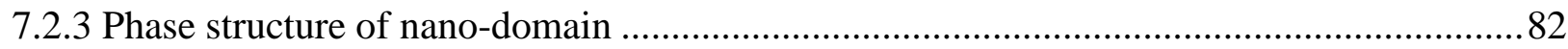

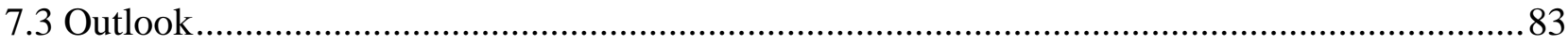

7.3.1 Nano-domain engineering vs. Piezoelectricity ........................................................ 83

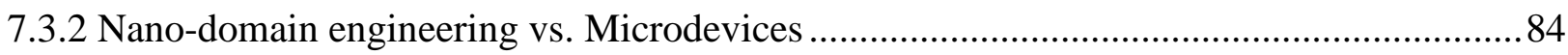

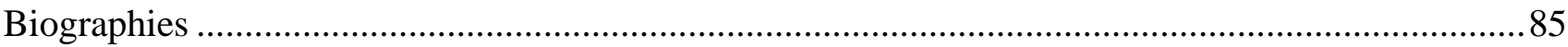

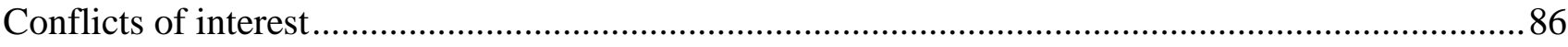

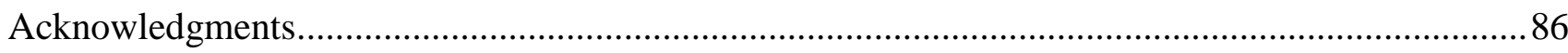

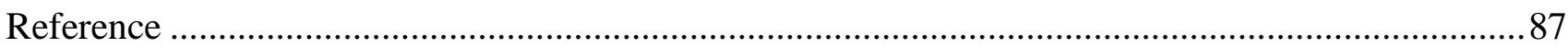




\section{Introduction}

Represented by lead zirconate titanate $\left\{(\mathrm{Pb}, \mathrm{Zr}) \mathrm{TiO}_{3}, \mathrm{PZT}\right\}$ family, piezoelectric materials, which can interconvert mechanical energy and electrical energy, are widely used in kinds of traditional electronic devices (including piezoelectric actuators, sensors, piezoelectric transducers and so on) and some new fields (including piezoelectric nanogenerators, medical equipment, and flexible devices). ${ }^{1-46}$ For the human health and environment protection, the usage or the content of toxic elements, such as lead $(\mathrm{Pb})$ and chromium $(\mathrm{Cr})$, is strictly restricted in these electronic devices. ${ }^{47-52}$ In particular, the renewal of the Restriction of the use of certain Hazardous Substances (e.g., RoHS 2) pointed out that “...information available does not allow excluding that lead-free solution for ceramics are or will become available within less than 5 years". ${ }^{12}$ Therefore, the research on lead-free piezoceramics, represented by potassium sodium niobate $\left\{(\mathrm{K}, \mathrm{Na}) \mathrm{NbO}_{3}, \mathrm{KNN}\right\}$, barium titanate $\left\{\mathrm{BaTiO}_{3}, \mathrm{BT}\right\}$, sodium bismuth titanate $\left\{\left(\mathrm{Bi}_{1 / 2} \mathrm{Na}_{1 / 2}\right) \mathrm{TiO}_{3}, \mathrm{BNT}\right\}$, and bismuth ferrite $\left\{\mathrm{BiFeO}_{3}, \mathrm{BFO}\right\}$, has become more important than ever, to replace lead-based counterparts. ${ }^{3-17,}$ 20, 21, 33-36, 40-45, 53-66

In this context, lead-free piezoelectric materials have been rapidly developed (see Figure 1(a)). Publications on lead-free piezoelectric materials increase extremely slow before 2004 due to the lack of promising performance comparable to those of lead-based ones. ${ }^{1,67-69}$ In 2004, Saito et al. obtained the high piezoelectricity (comparable to that of commercial soft PZT-4 ceramics) in LF4T ceramics by simultaneously using the reactive templated grain growth (RTGG) method and an orthorhombictetragonal (O-T) phase boundary. ${ }^{53}$ Their work ignited the researchers' passion for lead-free piezoceramics, resulting in a dramatic increase in lead-free piezoelectric materials' publication. Currently, publications on lead-free piezoelectric materials remain more than 350 per year. Meanwhile, 
these efforts have generated some eye-catching breakthroughs (see Figure 1(b)). Pure BT and KNN ceramics exhibit a much lower piezoelectric coefficient (e.g., $d_{33}<200 \mathrm{pC} / \mathrm{N}$ ) than those of PZT-based ceramics. Conversely, modified BT-, KNN-, BFO-based ceramics show comparable $d_{33}$ values than those of PZT-based ceramics. In particular, the application of phase boundary engineering (PBE) endows non-textured BT- and KNN-based ceramics with $d_{33}$ values as high as $490-700 \mathrm{pC} / \mathrm{N}, 54,55,59$, $60,65,66,70-72$ and the combination of RTGG method further increases KNN- and BT-based ceramics' $d_{33}$ values up to higher than $700 \mathrm{pC} / \mathrm{N} .{ }^{63,73}$ Figure $1(\mathrm{c})$ briefly summarizes the modification methods reported and their effects on lead-free piezoceramics' performance. If one uses one or more methods to modify these lead-free piezoceramics, the intrinsic and extrinsic contributions consequentially are affected, which definitively determine the obtained performance. ${ }^{74-82}$

(a)

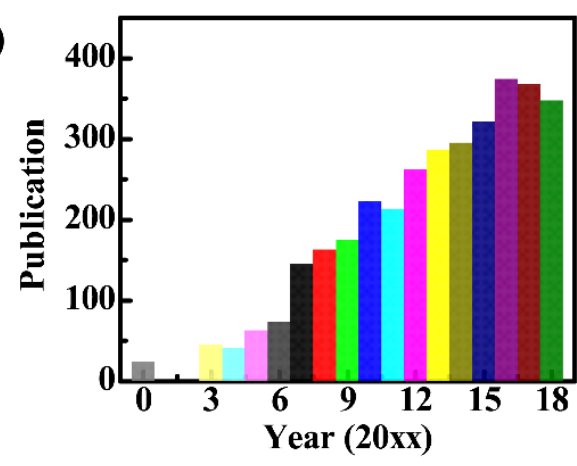

(c)

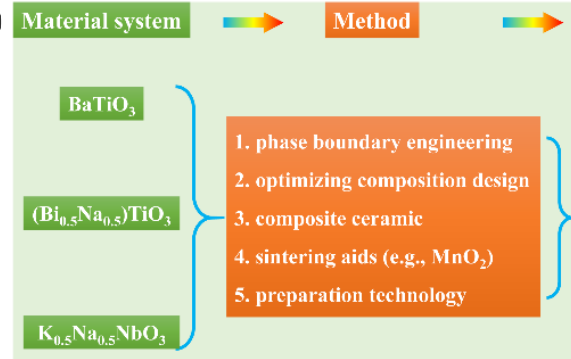

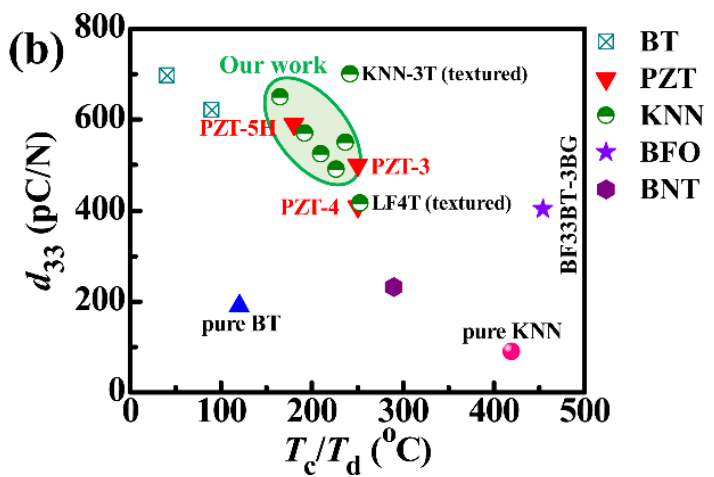

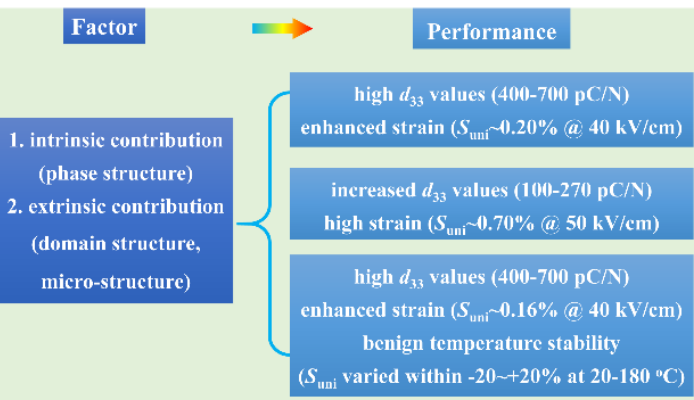

Figure 1. (a) Publication of lead-free piezoelectric materials varying with year. (b) $d_{33}$ and Curie temperature ( $T_{\mathrm{c}}$ ) (or depolarization temperature $T_{\mathrm{d}}$ for BNT ceramics). (c) Reported modification methods and their effects on lead-free piezoceramics' performance. The preparation technology includes the two-step sintering, ${ }^{83-88}$ RTGG method, ${ }^{53,63,73,}$ ${ }^{89-93}$ new sintering technology, ${ }^{94-96}$ poling conditions, ${ }^{97-106}$ sintering aids, ${ }^{107}$ and single crystal. ${ }^{78,82,86,108,109}$ 
When explaining piezo/ferroelectric properties of lead-free piezoceramics, both intrinsic and extrinsic contributions are considered. ${ }^{74-77,80-82,110,111}$ The intrinsic contribution is related to the lattice arrangement (e.g., lattice distortion), ${ }^{112-129}$ while the extrinsic one mainly originates from the microstructure, including ferroelectric domains, grain size, density, and porosity. ${ }^{130-145}$ If crushing a piezoceramic, one can obtain countless grains (see Figure 2). These grains are comprised of numerous complicated ferroelectric domains that are the consequence of the lattice arrangement. Therefore, the domain structure acts as a bridge connecting the lattice alignment with macro grains (or ceramics). Generally, ferroelectric domains are classified into three types according to their scales, that is, microndomains, nano-domains, and polar nanoregions (PNRs). In particular, nano-domains and PNRs are widely reported to play an important role in the performance enhancement in both lead-based and leadfree piezoceramics because of their faster switching than micron-domains. ${ }^{58-63,82,146-149}$ From the view of applications, the extrinsic contributions are important, as they are responsible for nonlinearity (field dependence of the piezoelectric properties), weak-field hysteresis, frequency dispersion of the properties, creep, fatigue behavior, and aging. ${ }^{76}$

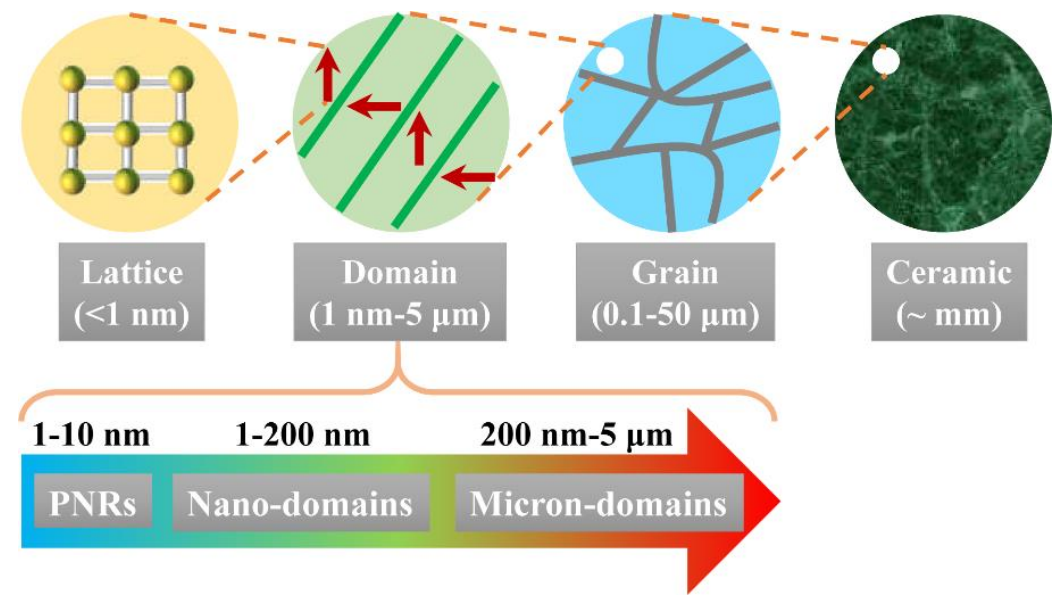

Figure 2. A schematic diagram describing the structure of a piezoceramic at different scales. 
The systematic observation and investigation of nano-domains were started with lead-based piezoceramics. In 2007, Schmitt et al. systematically revealed the domain structure of the $\mathrm{PbZrO}_{3}-$ $x \mathrm{PbTiO}_{3}$ solid solution (see Figure 3). ${ }^{141,142,150}$ All three compositions with representative phase structures (e.g., rhombohedral, R, rhombohedral-tetragonal, R-T, and tetragonal, T) exhibited the bimodal micron-domain distribution, showing the alternative broad and narrow domain width. In the R phase region, $0.60 \mathrm{PbZrO}_{3}-0.40 \mathrm{PbTiO}_{3}$ ceramics exhibited the wavy domain walls and possessed areas with lamellar domain configurations with an irregular distribution of narrow and broad domain widths (see Figure 3(b)). When locating at the morphotropic phase boundary (MPB) region, $0.525 \mathrm{PbZrO}_{3}-0.475 \mathrm{PbTiO}_{3}$ ceramics exhibited decreased bimodal micron-domain distribution and the occurrence of nano-domains (see Figure 3(c)). These nano-domains aligned within the microndomains hierarchically and possessed an angle of $\sim 45^{\circ}$ with respect to the micron-domains (marked by the red arrows). In the $\mathrm{T}$ phase region, $0.45 \mathrm{PbZrO}_{3}-0.55 \mathrm{PbTiO}_{3}$ ceramics only exhibited the alternative lamellar domains with a scale of $66 \mathrm{~nm}$ for narrow domains and $147 \mathrm{~nm}$ for broad ones (see Figure 3(d)). Therefore, the complex domain structure was believed to play an important role in the piezoelectricity enhancement at MPB. ${ }^{141,142,150}$ After then, abundant investigations were carried out on the ferroelectric domains of $\mathrm{PZT}$ and relaxor- $\mathrm{PbTiO}_{3}$ (e.g., $\mathrm{Pb}\left(\mathrm{Mg}_{1 / 3} \mathrm{Nb}_{2 / 3}\right) \mathrm{O}_{3}-\mathrm{PbTiO}_{3}, \mathrm{PMN}-\mathrm{PT}$ and $\mathrm{Pb}\left(\mathrm{Zn}_{1 / 3} \mathrm{Nb}_{2 / 3}\right) \mathrm{O}_{3}-\mathrm{PbTiO}_{3}, \mathrm{PZN}-\mathrm{PT}$ ) piezoelectric materials, even the in situ (real-time) observations under external stimulus (e.g., electric field and temperature). ${ }^{143-145,151-153}$ The results observed strongly suggested that the domain switching and domain wall motion significantly affected the macro performance including piezoelectricity, ferroelectricity, temperature stability, aging, and fatigue behavior, specifically the occurrence of nano-domains and PNRs. ${ }^{129,}$ 137-145, 149-154 


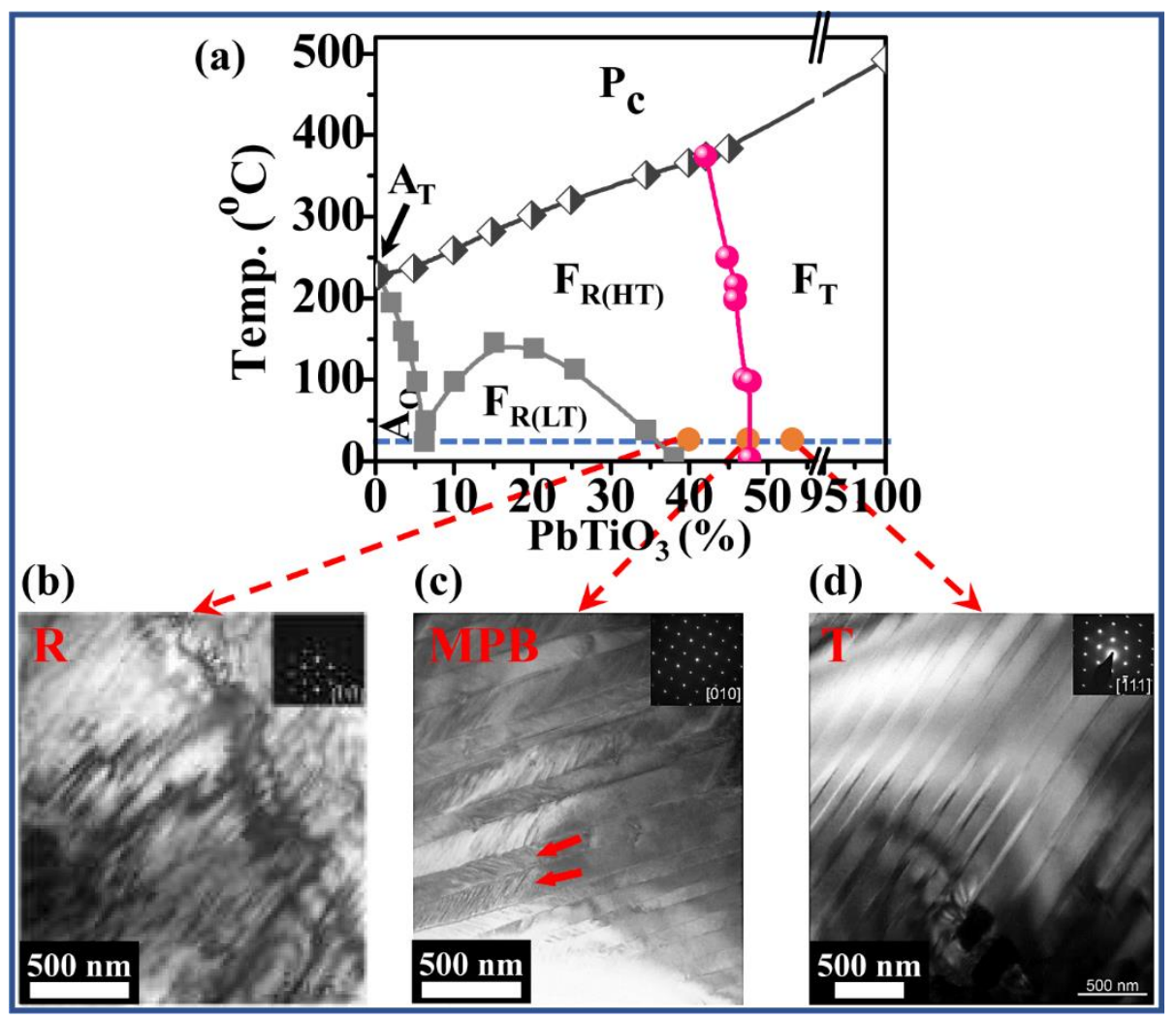

Figure 3. (a) Phase diagram of $\mathrm{PbZrO}_{3}-x \mathrm{PbTiO}_{3}$ solid solution; Domain configuration of (b) $0.6 \mathrm{PbZrO}_{3}-0.4 \mathrm{PbTiO}_{3}$, (c) $0.525 \mathrm{PbZrO}_{3}-0.475 \mathrm{PbTiO}_{3}$, and (d) $0.45 \mathrm{PbZrO}_{3}-0.55 \mathrm{PbTiO}_{3}$ ceramics. (a-d) Reproduced from ref. 141. with permission from the American Institute of Physics, Copyright 2007.

Although investigations on ferroelectric domains of lead-free piezoceramics were started in the 1980s, it was not until 2005 that the substantial progress was achieved due to the limitation of observation technology. ${ }^{135,136,155}$ Moreover, the early investigations mainly concentrated on observing the ferroelectric domains of pristine lead-free piezoceramics, such as pure $\mathrm{BaTiO}_{3}$ and $\left(\mathrm{K}_{0.5} \mathrm{Na}_{0.5}\right) \mathrm{O}_{3}$ ceramics, because of the lack of high-performance lead-free piezoceramics. ${ }^{152,155,156}$ These results only help understand the basic ferroelectric domain configuration that could be predicted by the theoretical calculation, but cannot promote the understanding and design of high-performance leadfree piezoceramics. Recently, the PBE endows lead-free piezoceramics with comparable performance 
to part commercial lead-based ceramics. ${ }^{54-62,}$ 64-66, 70, 157 These high-performance lead-free piezoceramics make it meaningful and useful to study their ferroelectric domains to understand the related physical mechanisms, which could help further design lead-free piezoceramics in return. In particular, the recent results from lead-free ceramics with PBE strongly demonstrated the crucial role of nano-domains and PNRs in the piezoelectricity enhancement. ${ }^{56-66,158}$ Previous reviews mainly

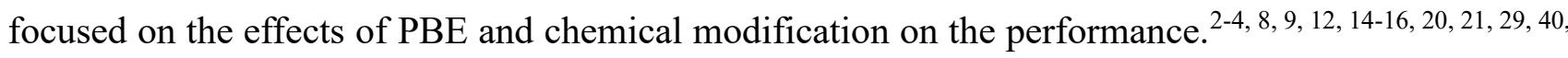
41, 159 They generally attributed the enhanced performance to the phase boundary and the domain structure without summarizing and analyzing the domain structure in depth. In particular, most of these reviews have dealt with the development of a specific kind of lead-free piezoelectric family, so that discussions of nano-domains and PNRs were not well mentioned, or only covered as a minor part of a larger field. Recently, the developed characterization techniques, represented by the transmission electron microscope (TEM) and piezoresponse force microscope (PFM), allow us to observe the domain structure more easily, even under the external stimulus (e.g., electric field and temperature). Owing to these technologies, the recent publications show a substantial advance in analyzing the ferroelectric domains of high-performance lead-free piezoceramics, such as the observation of nanodomains and PNRs, as well as domains varying with the external stimulus. Considering the important role of domain structure in piezoceramics and driven by the demand of replacing the lead-based piezoceramics, it is necessary to present a review to summarize the development of domain structure of lead-free piezoceramics and analyze the related physical mechanisms in depth, particularly focusing on the recently discovered nano-domains and PNRs, which is the main motivation for this review. Figure 4 shows the structure chart of this review. In this review, we first introduced the foundation of domain structure and then presented the observation tools for domain structure. We then summarized 
the variations of nano-domains PNRs with phase structure, electric field, and temperature, as well as their effects on the macro performance (e.g., piezoelectricity, strain, temperature stability, fatigue, and aging), focusing on the related physical mechanisms. Finally, we gave out the challenges and outlook of nano-domains and PNRs of lead-free piezoceramics.

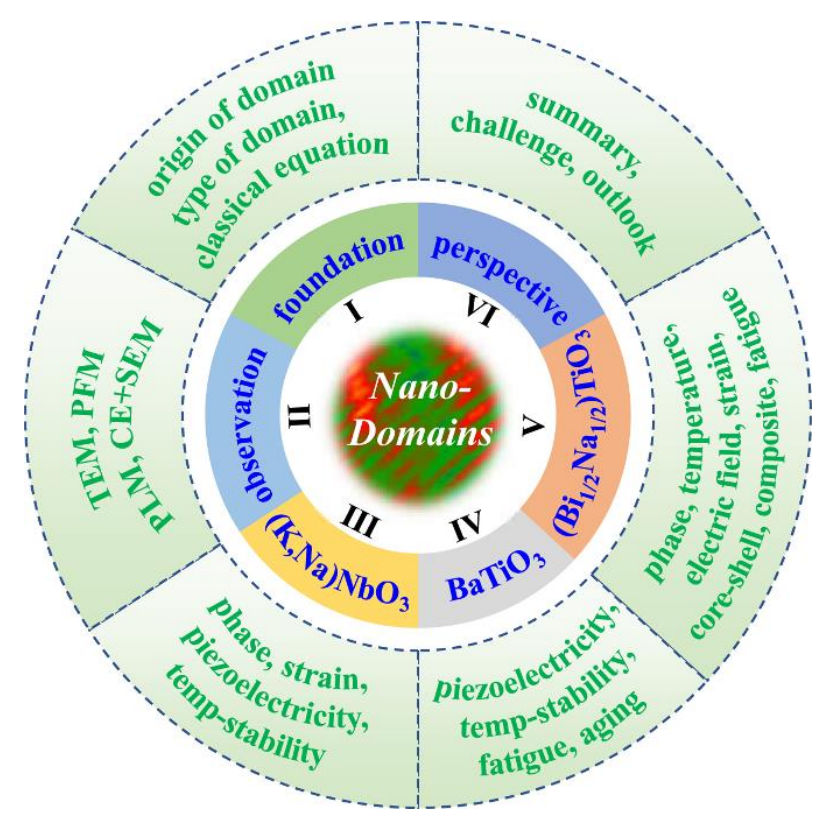

Figure 4. Structure chart of this review. The center image is a colored picture of a typical nano-domain with a scale of $\sim 30 \mathrm{~nm}$, and the Roman numerals indicate the order of the contents.

\section{Foundation of domain}

An area, in which the spontaneous polarization $\left(P_{\mathrm{s}}\right)$ vectors align with the same direction, is called a domain, and the boundary separating two adjacent domains is termed as a domain wall (see Figure 5(a)). ${ }^{134,135}$ The theory suggests that the formation of domains in ferroelectrics is the consequence of the reduction in the elastic and electrostatic energies, which has been largely discussed in previous publications. ${ }^{41}$ As we know, the domain structure strongly depends on the phase structure. Pure KNN and BT ceramics exhibit similar variations in phase structure when decreasing temperature from their $T_{\mathrm{c}}$ values, that is, Cubic $(C) \rightarrow$ Tetragonal $(T) \rightarrow$ Orthorhombic $(O) \rightarrow$ Rhombohedral $(R) .{ }^{6,9,41}$ For pure 
T, O, and R phases, the permitted numbers of $P_{\mathrm{s}}$ vectors are respectively 6,12 , and $8,{ }^{144}$ as simulated in Figure 5(b-d). Therefore, the permitted domain structures are $90^{\circ}$ and $180^{\circ}$ domains for a pure $\mathrm{T}$ phase, are $60^{\circ}, 120^{\circ}, 90^{\circ}$, and $180^{\circ}$ domains for a pure $O$ phase, and are $71^{\circ}, 109^{\circ}$, and $180^{\circ}$ domains for a pure R phase (see Figure 5(b-d)). ${ }^{144}$ Although the domain structure of a pure phase structure is relatively easy, the real domain structure in a piezoceramic is much more complicated due to the interplay of these domains. That is the reason why even a pure KNN ceramic with a single O phase still exhibits the complicated domain structure. ${ }^{152,156}$ Generally, both $\mathrm{KNN}$ - and BT-based ceramics without adding overmuch additives exhibit the long-range ordered domain structure. ${ }^{136,152,155,156,160-}$ ${ }^{162}$ While BNT-based ceramics usually display a coexistence of long-range ordered domains and nanodomains, and the chemical modification usually tends to increase the content of nano-domains and thus increases the relaxor degree. ${ }^{163-166}$ The basic variation in grain size affects the domain size, and from the balance of the depolarization energy and the domain wall energy, the relationship between the domain size $(d)$ and the grain size $(g)$ can be expressed as

$$
d=\left\{\left(\frac{\sigma}{\varepsilon^{*} P_{o}^{2}}\right) g\right\}^{1 / 2}
$$

where $\sigma, P_{\mathrm{o}}$, and $\varepsilon^{*}$ are the energy density of the domain wall, the spontaneous polarization, and the effective dielectric constant, respectively. ${ }^{168}$ Thus, the domain size strongly depends on the grain size, which was largely demonstrated in perovskite-structure ferroelectrics. While the subsequent experimental observations suggested that the domain size cannot increase monotonously with the increasing grain size due to the free energy of the system. ${ }^{67,134}$ From the view of microstructure, an empirical equation is generally followed in ferroelectric ceramics, ${ }^{169}$

$$
d \propto g^{m}
$$

where $g$ represents the grain size, and $m$ is an exponent. When $g$ is within 1-10 $\mu \mathrm{m}, m$ approaches to 
$1 / 2 ; g$ is larger than $10 \mu \mathrm{m}, m$ is within $0-1 / 2$, and $g$ is smaller than $1 \mu \mathrm{m}, m$ is within $1 / 2-1 .^{169}$ It is easy to understand this empirical equation. When progressively reducing the grain size, the whole grain will only contain one domain or even less one domain, indicating $m$ approaches to 1 . When monotonously increasing the grain size, the domain cannot follow a monotonous increase due to the free energy of the system, the internal stress, and the coupling of domains and grains, resulting in an $m$ less than $1 / 2$.

(a)

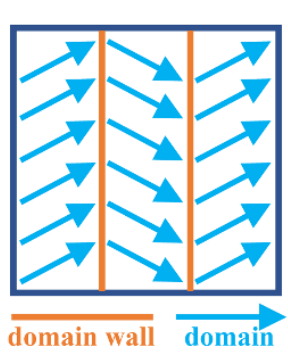

(d)

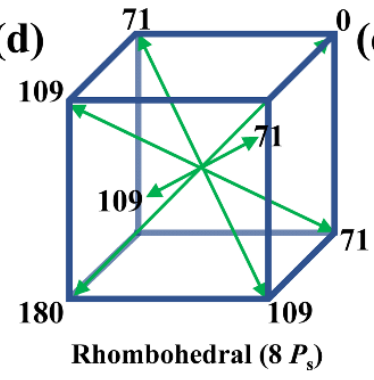

(b)

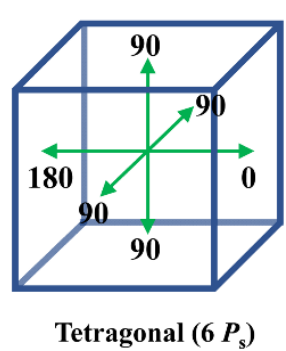

(e)

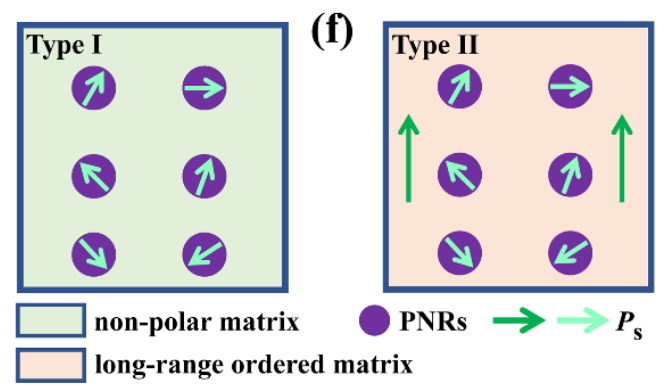

Figure 5. (a) Schematic diagram of the domain and domain wall. The direction of $P_{\mathrm{s}}$ in (b) tetragonal, (c) orthorhombic, and (d) rhombohedral phase. Angles between polarization direction "0" and its symmetry equivalent ones are indicated. ${ }^{144}(\mathrm{e}, \mathrm{f})$ Schematic diagrams of PNRs in two different relaxor situations. ${ }^{66}$ (e, f) Reproduced from ref. 66. with permission from the American Chemical Society, Copyright 2019.

Generally, PNRs are believed to originate from the local structural heterogeneity that is induced by the nanoscale fluctuation of dipoles, which endow the ferroelectrics with the obvious frequency dependence of dielectric properties (i.e., relaxor behavior). ${ }^{18,78-80,82,147,170-215}$ According to the surrounding matrix, two types of PNRs are generally observed (see Figure 5(e, f)). ${ }^{66}$ In type I, PNRs 
are embedded into a non-polar matrix and its relaxor behavior occurs at the paraelectric-ferroelectric phase transition. The type I is widely observed in $\mathrm{PbMg}_{1 / 3} \mathrm{Nb}_{2 / 3} \mathrm{O}_{3}(\mathrm{PMN})$ and $(\mathrm{Pb}, \mathrm{La})(\mathrm{Zr}, \mathrm{Ti}) \mathrm{O}_{3}(\mathrm{PLZT})$ relaxor ferroelectrics and deemed as the conventional relaxor state. ${ }^{170,172,179,183-185}$ In type II, the matrix is polar and exhibits abundant long-range ordered ferroelectric domains. The relaxor behavior of type II occurs away from the ferroelectric to ferroelectric and paraelectric to ferroelectric phase transitions. Type II is usually observed in a relaxor-ferroelectric solid solution, such as $\mathrm{PbMg}_{1 / 3} \mathrm{Nb}_{2 / 3} \mathrm{O}_{3}-\mathrm{PbTiO}_{3}(\mathrm{PMN}-\mathrm{PT})$ and $\mathrm{PbZn}_{1 / 3} \mathrm{Nb}_{2 / 3} \mathrm{O}_{3}-\mathrm{PbTiO}_{3}(\mathrm{PZN}-\mathrm{PT}) .{ }^{78-80,}$ 82, 147, 171, 187-189, 202 For BT- and KNN-based lead-free piezoceramics, the application of PBE is to construct the multi-phase coexistence near room temperature by introducing some necessary additives into pristine $\mathrm{BaTiO}_{3}$ and $\mathrm{K}_{0.5} \mathrm{Na}_{0.5} \mathrm{NbO}_{3}$ ceramics that possess the long-range ordered ferroelectric domains. ${ }^{9,}$ 14, 41, 42 Therefore, the relaxor behavior of BT- and KNN-based ceramics with PBE occurs along with the ferroelectricferroelectric phase transition at room temperature, which is different from the aforementioned two types, as shown in Figure 6. As for BNT-based ceramics, the application of PBE usually changes the domains into the nano-domains or even non-polar matrix. ${ }^{216}$ Therefore, its relaxor behavior mainly originates from the nano-domains. ${ }^{163}$ Previously, considerable efforts have been given on the basic understanding of ferroelectric domains of pristine lead-free piezoceramics showing the moderate performance. ${ }^{136,152,156,217-219}$ However, the recent results from lead-free ceramics with PBE strongly demonstrated the crucial role of nano-domains and PNRs in the performance enhancement, which was not well summarized and understood in previous reviews. In this review, we thus focus on summarizing and discussing the effects of nano-domains and PNRs on the performance of KNN-, BT-, and BNTbased ceramics. 

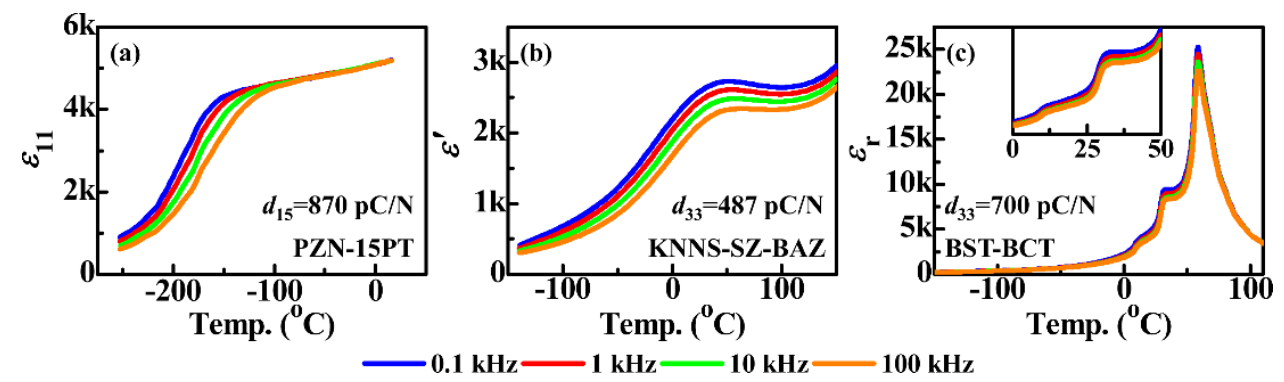

Figure 6. Temperature-dependent dielectric permittivity of (a) PZN-15PT single crystal, ${ }^{78}$ (b) KNNS-SZ-xBAZ $(x=0.035)$ ceramics, ${ }^{158}$ and (c) BS0.11T- $x$ BCT $(x=0.18)$ ceramics ${ }^{65}$ The relaxor behavior of PZN-15PT occurs at $T \leq-$ $100{ }^{\circ} \mathrm{C}$, and $\mathrm{KNN}$ - and BT-based ceramics with PBE exhibit the relaxor behavior near room temperature. (a) Reproduced from ref. 78. with permission from the Springer Nature, Copyright 2016; (b) Reproduced from ref. 158. with permission from the Royal Society of Chemistry, Copyright 2019; (c) Reproduced from ref. 65. with permission from the American Chemical Society, Copyright 2018.

\section{Observation tools of domain}

Before discussing the effects of nano-domains and PNRs on lead-free piezoceramics' performance, we briefly introduced the currently used tools for domain observation. At present, four methods are used to observe the domain structure, that is, chemical etching (CE), TEM, PFM, and polarized light microscopy (PLM). ${ }^{13,220}$ Previous books and other publications have described these methods. ${ }^{13,220}$ Here we mainly compared the four methods concerning different factors, as listed in Table 1. Generally, the domains exposed by the chemical etching are observed by a scanning electron microscope (SEM). ${ }^{87}$, 88, 131, 221-223 Among these methods, TEM and PFM show the remarkable ability to observe and manipulate domain structure, which are widely adopted to detect the domain structure of piezoceramics. ${ }^{220,224,225}$ In particular, both two methods allow one to record the variations of domain structure under the external stimulus (e.g., electric field and temperature), greatly benefitting for analyzing the physical mechanisms. CE+SEM is also used to observe the domain structure of 
piezoceramics due to the low cost and easy operability. But this method inevitably and irreversibly destroys the domain structure and erases some subtle domains (e.g., nano-domains) to some degree. Therefore, $\mathrm{CE}+\mathrm{SEM}$ is a good option for observing the large domain structure, such as microndomains. Besides, PLM is generally adopted for detecting the domain structure of a piezo/ferroelectric single crystal. ${ }^{226-229}$

Table 1. Comparison of four domain observation methods.

\begin{tabular}{llllcc}
\hline Method & Resolution & Cost & Operability & in $^{\text {situ }}$ observation & Destruction \\
\hline TEM & High & High & Difficult & Yes & No \\
PFM & Relatively high & Moderate & Relatively difficult & Yes & No \\
PLM & Low & Low & Moderate & Yes & No \\
CE+SEM & Moderate & Low & Easy & No & Yes \\
\hline
\end{tabular}

* "in situ observation" means that "Can this method do in situ observation for domains under an external stimulus (e.g., electric field and temperature)?"

\section{Nano-domain in KNN-based ceramics}

KNN-based ceramics have attracted the researchers' attention because of the promising performance comparable to that of lead-based ceramics. ${ }^{53,55,59-64,66,146,154,157,158,230-275}$ In particular, the new PBE proposed by Wu et al, simultaneously shifting the rhombohedral-orthorhombic and orthorhombictetragonal phase transition temperatures $\left(T_{\mathrm{R}-\mathrm{O}}\right.$ and $\left.T_{\mathrm{O}-\mathrm{T}}\right)$ to room temperature, markedly increased nontextured KNN-based ceramics' $d_{33}$ values up to $400-650 \mathrm{pC} / \mathrm{N}$, even higher than that of commercial soft PZT-4 ceramics. ${ }^{55,60-64,66,90,92,93,146,157,158,230-240,266,276-284}$ At the same time, KNN-based ceramics

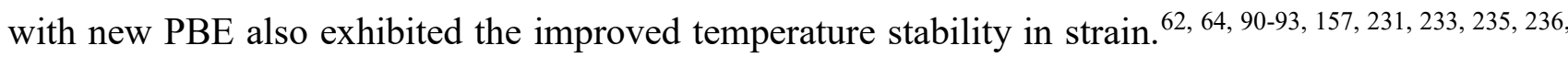
239, 262, 264, 266, 280, 284-297 The analysis demonstrated that the performance enhancement mainly originated from the multi-phase coexistence and the complex domain structure including micron-domains, nano- 
domains, and PNRs. Therefore, the effects of nano-domains and PNRs on KNN-based ceramics are discussed in this section.

\subsection{Nano-domain vs. Phase structure}

Figure 7 shows the domain structure of KNN-based ceramics with a single O phase. ${ }^{152,160,233}$ The micron-domains were observed in these ceramics. Pure KNN ceramics displayed the regular domain structures with the shapes of "herringbone" and "watermark" (see Figure $7(a-c)) .{ }^{152}$ The herringbone domains were regarded as $90^{\circ}$ domains, and the watermark ones represented $180^{\circ}$ domains. These domains generally exhibited a size of micrometer scale, that is, $0.5-1 \mu \mathrm{m}$ in width and 3-7 $\mu \mathrm{m}$ in length for $90^{\circ}$ domains, and 3-10 $\mu \mathrm{m}$ in width and $20-40 \mu \mathrm{m}$ in length for $180^{\circ}$ domains. The similar herringbone domains were also found in $\mathrm{KNN}-\mathrm{MnO}_{2}$ ceramics by the PFM measurements (see Figure $7(\mathrm{e}, \mathrm{f})) .{ }^{160}$ The difference is that $\mathrm{KNN}-\mathrm{MnO}_{2}$ ceramics displayed the smaller domains because of adding $\mathrm{MnO}_{2}$ sintering aid, accompanying with a length of $1-2 \mu \mathrm{m}$ and a width of $0.4-0.6 \mu \mathrm{m}$. The observed regular domain structures in pure $\mathrm{KNN}$ ceramics were determined by the intrinsic symmetry of the $\mathrm{O}$ phase, as discussed above. Besides the regular domains, the irregular domains were also observed in KNN-based ceramics with a single O phase, such as KNNS-SZ ceramics (see Figure 7(d)) and KNN ceramics synthesized at different sintering temperatures. ${ }^{156,218,233}$ The irregular domains exhibited a scale of $0.5-2 \mu \mathrm{m}$ and were believed to arise from the addition of additives (e. g., $\mathrm{Sb}^{5+}$ and $\mathrm{SrZrO}_{3}$ ) and the microstructure (e.g., grain size and element distribution). ${ }^{218,}{ }^{233}$ As expected, the relatively moderate $d_{33}$ values of $100-200 \mathrm{pC} / \mathrm{N}$ were also found in these ceramics because of the single O phase. ${ }^{152,156,160,218,219,233,298}$ 

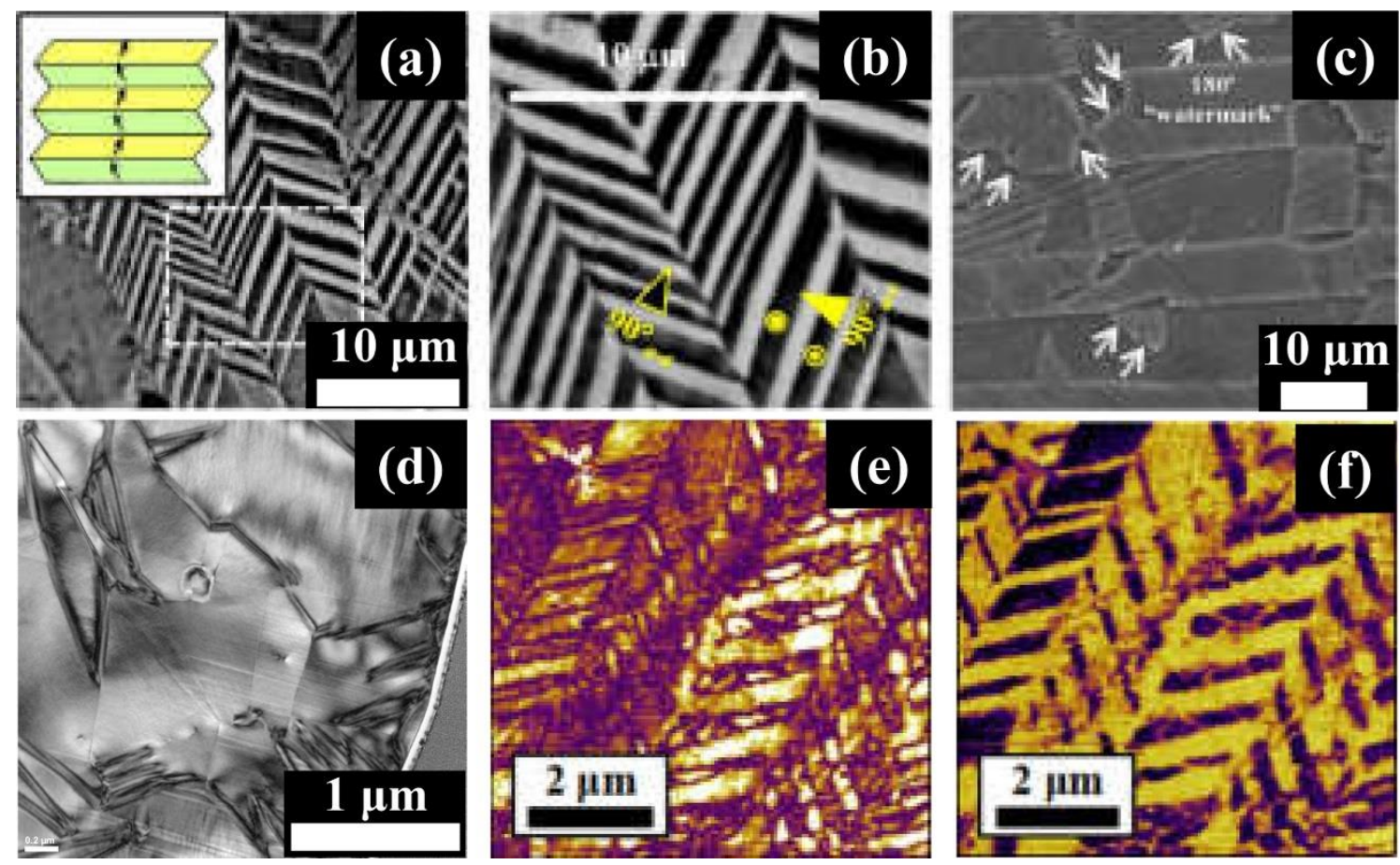

Figure 7. Domain structure of unpoled KNN-based ceramics with a single $\mathrm{O}$ phase. (a-c) $\mathrm{K}_{0.5} \mathrm{Na}_{0.5} \mathrm{NbO}_{3}$ ceramics; ${ }^{152}$

(d) $0.99 \mathrm{~K}_{0.5} \mathrm{Na}_{0.5} \mathrm{Nb}_{0.965} \mathrm{Sb}_{0.035} \mathrm{O}_{3}-0.01 \mathrm{SrZrO}_{3}$ (KNNS-SZ) ceramics; ${ }^{233}$ (e) piezoresponse image and (f) phase of $\left(\mathrm{K}_{0.45} \mathrm{Na}_{0.55}\right) \mathrm{NbO}_{3}-0.2 \% \mathrm{MnO}_{2}$ ceramics. ${ }^{160}$ (a-c) Reproduced from ref. 152. with permission from the Elsevier, Copyright 2011; (d) Reproduced from ref. 233. with permission from the Royal Society of Chemistry, Copyright 2019; (e, f) Reproduced from ref. 160. with permission from the American Institute of Physics, Copyright 2019.

To improve the piezoelectric properties of KNN ceramics, the PBE and the chemical modification were used to modify pure KNN ceramics, resulting in the distinct variations of domain structure. Figure 8 displays the domain structure of represented KNN-based ceramics with multi-phase coexistence before poling. ${ }^{60-64,66,87,88,90,93,131,146,151,158, ~ 221-223, ~ 231, ~ 233, ~ 264, ~ 276, ~ 292, ~} 299$ Compared to the domains of the single $\mathrm{O}$ phase, both the size and the shape of domain structures are significantly changed in the multi-phase coexistence. Firstly, the multi-phase coexistence showed the miniaturized domains with an average size of smaller than $0.5 \mu \mathrm{m}$. Of particular importance is that KNN-based ceramics with RO-T/R-T phase coexistence even exhibited the nanoscale domains. The miniaturized domains respond 
to the external stimulus (e.g., electric field and stress) more easily than the large counterparts, thus promoting the piezoelectric properties. ${ }^{134,142,153,300-302}$ Then, the regular domain structures were replaced by complex ones. KNN-based ceramics with O-T phase coexistence exhibited the striped and curved domains with an average scale of $200 \mathrm{~nm}$ (see Figure 8(a1-a3)). ${ }^{264,292}$ Furthermore, Zuo et al. found that part striped domains in the O-T phase coexistence were comprised of alternate and hierarchical nano-domains with a scale of $20 \mathrm{~nm}$ and $50 \mathrm{~nm} \cdot{ }^{151}$ Convergent beam electron diffraction (CBED) patterns demonstrated that these alternate nano-domains possessed an O phase or a $\mathrm{T}$ phase. ${ }^{151}$

Furthermore, KNN-based ceramics with R-O-T/R-T phase coexistence exhibited the more complicated domain structures compared to the ones with $\mathrm{O}$ phase and $\mathrm{O}-\mathrm{T}$ phase coexistence, accompanying with the shapes of striped, curved, watermarks, short segments, tweed-like, and nanoscale (see Figure 8(b)). ${ }^{59,60,62,64,158,276}$ The CE+SEM method unveiled the abundant short "segments" domains and “watermark" domains in KNNS-BNKZ ceramics with R-T phase coexistence (see Figure 8(b1-b2)). ${ }^{276}$ The short domain segments were ascribed to the R-T phase coexistence and the existence of $180^{\circ}$ ferroelectric domain walls, and the watermark domains were related to the rhombohedral $180^{\circ}$ ferroelectric domain walls. ${ }^{276,303,304}$ KNNS-BNKH ceramics with R-T phase coexistence exhibited the striped domains with a scale of 50-200 nm that were comprised of hierarchical nano-domains with a scale of 10 30 nm (see Figure $8(\mathrm{~b} 3)$ ). ${ }^{62}$ The hierarchical nano-domains were also reported in other high-performance piezoelectric materials, such as 50BZT-50BCT, MPB-featured PZT, and relaxor$\mathrm{PbTiO}_{3}$ ceramics or single crystals, and believed to be responsible for the piezoelectricity enhancement. ${ }^{137,138,140-143,145,150,305,306}$ Both KNNS-BZ-BNH-MnO 2 and KNNS-SZ-BAZ ceramics displayed the striped nano-domains and the rectangular-ambulatory domains composed of striped and 
tweed-like nano-domains (see Figure 8(b4-b7)). ${ }^{64,158}$ More importantly, KNNS-BZ-BKH and KNNSBF-BNZ ceramics, which possessed an ultra-high piezoelectricity (e.g., $d_{33}=550-570 \mathrm{pC} / \mathrm{N}$ ), universally displayed the ultra-fine domains with the scale of $1.8 \mathrm{~nm}$ (see Figure $8(\mathrm{~b} 8-\mathrm{b} 11)$ ). ${ }^{59,60}$

The complex domain configurations in PBE-modified KNN-based ceramics mainly originate from the multi-phase coexistence that permits more possible $P_{\mathrm{s}}$ variants (see Figure 5). Furthermore, polarization variants of different phases interact with each other, resulting in the complicated domain configurations. From the view of free energy, the domain size is also reduced as the consequence of the flattened energy potential in the multi-phase coexistence region. ${ }^{134,135,305}$ The instability of multiphase coexistence results in the low polarization anisotropy that leads to the decrease in domain wall energy and consequently reduces the domain size. ${ }^{134,135,305,307,308}$ Subsequently, the miniatured domains easily respond to the electric field, promoting the macro performance of KNN-based ceramics. Besides the multi-phase coexistence, the aliovalent substitutions caused by the indispensable additives also break long-range ordering of the ferroelectric matrix, preventing the formation of long-range ordered domains and inducing the nano-domains (or even PNRs). ${ }^{309}$ Therefore, the nano-domains in KNN-based ceramics are induced by both the multi-phase coexistence and the destruction of longrange ordering, differing from PZT ceramics whose nano-domains are only induced by the R-T coexistence phase. ${ }^{150}$ Thus, the trade-off relationship between the long-range ordering and nanodomains (specially PNRs) occurs on improving the piezoelectric properties of KNN-based ceramics, which is discussed in the subsequent section of PNRs. 


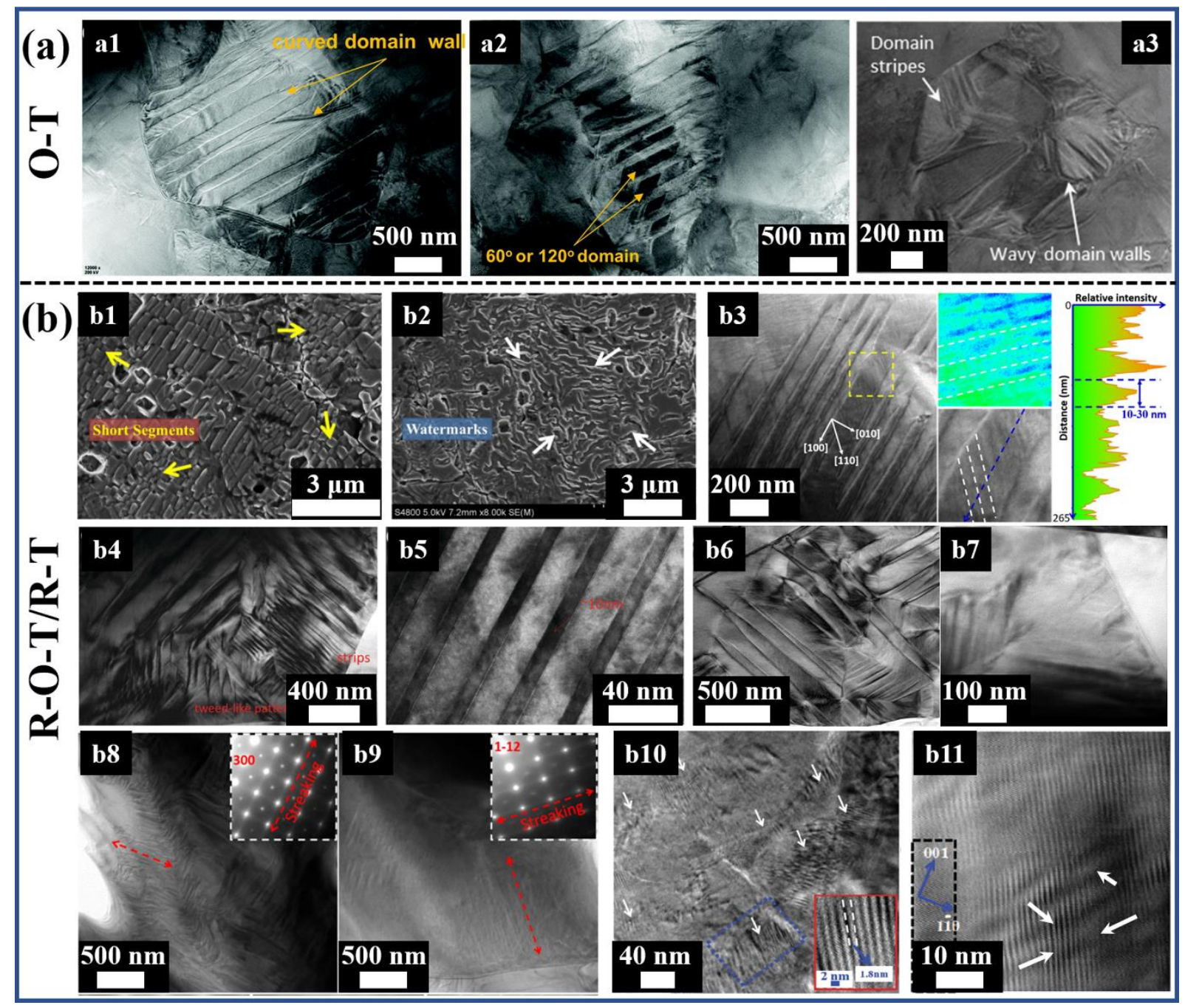

Figure 8. Nano-domains of unpoled KNN-based ceramics with multi-phase coexistence. Nano-domains of KNN-

based ceramics with (a) O-T and (b) R-O-T/R-T phase coexistence. (a1, a2) KNNL-BZ-BNT ceramics, ${ }^{264}$ (a3) KNNSL-BNZ-BZ-MnO 2 ceramics, ${ }^{292}$ (b1, b2) KNNS-BNKZ ceramics, ${ }^{276}$ (b3) KNNS-BNKH ceramics, ${ }^{62}$ (b4, b5) KNNS-BZ-BNH-MnO 2 ceramics,${ }^{64}$ (b6, b7) KNNS-SZ-BAZ ceramics, ${ }^{158}$ (b8, b9) KNNS-BF-BNZ ceramics, ${ }^{59}(\mathrm{~b} 10$, b11) KNNS-BZ-BKH ceramics. ${ }^{60}$ (a1, a2) Reproduced from ref. 264. with permission from the Royal Society of Chemistry, Copyright 2018; (a3) Reproduced from ref. 292. with permission from the Royal Society of Chemistry, Copyright 2018; (b1, b2) Reproduced from ref. 276. with permission from the American Chemical Society, Copyright 2016; (b3) Reproduced from ref. 62. with permission from the Royal Society of Chemistry, Copyright 2017; (b4, b5) Reproduced from ref. 64. with permission from the Royal Society of Chemistry, Copyright 2018; (b6, b7) Reproduced from ref. 158. with permission from the Royal Society of Chemistry, Copyright 2019; (b8, b9) Reproduced from ref. 
59. with permission from the American Chemical Society, Copyright 2016; (b10, b11) Reproduced from ref. 60. with permission from the Wiley-VCH, Copyright 2016.

\subsection{Nano-domains vs. Piezoelectricity}

To further reflect the effects of nano-domains on KNN-based ceramics, we collected the reported domain structure in unpoled KNN-based ceramics, as listed in Table 2. The domain structure depends on not only the phase structure but also the chemical design. It is not surprising to obtain this conclusion because the domain structure is determined by the aligned $P_{\mathrm{s}}$ vectors that rely on the phase structure and the chemical composition. Furthermore, KNN-based ceramics with multi-phase coexistence exhibited higher $d_{33}$ values compared to the ones with a single-phase structure because of the reduced domain size and multi-phase coexistence. In particular, KNN-based ceramics with nanodomains generally exhibited $d_{33}$ values higher than $400 \mathrm{pC} / \mathrm{N}$. The relationship between domain size

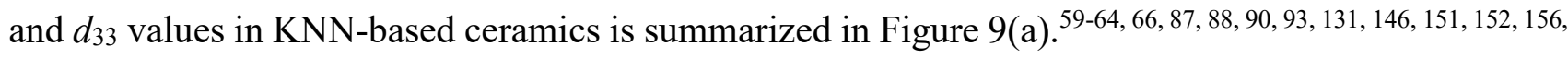
$158,160,218,219,221-223,231,233,264,276,292,298,299,310$ An intuitional tendency is that the smaller domain is, the higher $d_{33}$ is. Intriguingly, the occurrence of nano-domains significantly increased $d_{33}$ values up to 490 $700 \mathrm{pC} / \mathrm{N}$. Generally, it is believed that nano-domains and PNRs are prone to switching under an electric field, promoting the net piezoelectricity. To prove this, we collected the coercive field $\left(E_{\mathrm{c}}\right)$ of KNN-based ceramics with nano-domains and their counterparts without nano-domains (see Figure 9(b)). ${ }^{59,60,62,64,146,158,230,233}$ The occurrence of nano-domains visibly reduced the $E_{\mathrm{c}}$ of KNN-based ceramics. 
Table 2. Reported domain structure in unpoled KNN-based ceramics*.

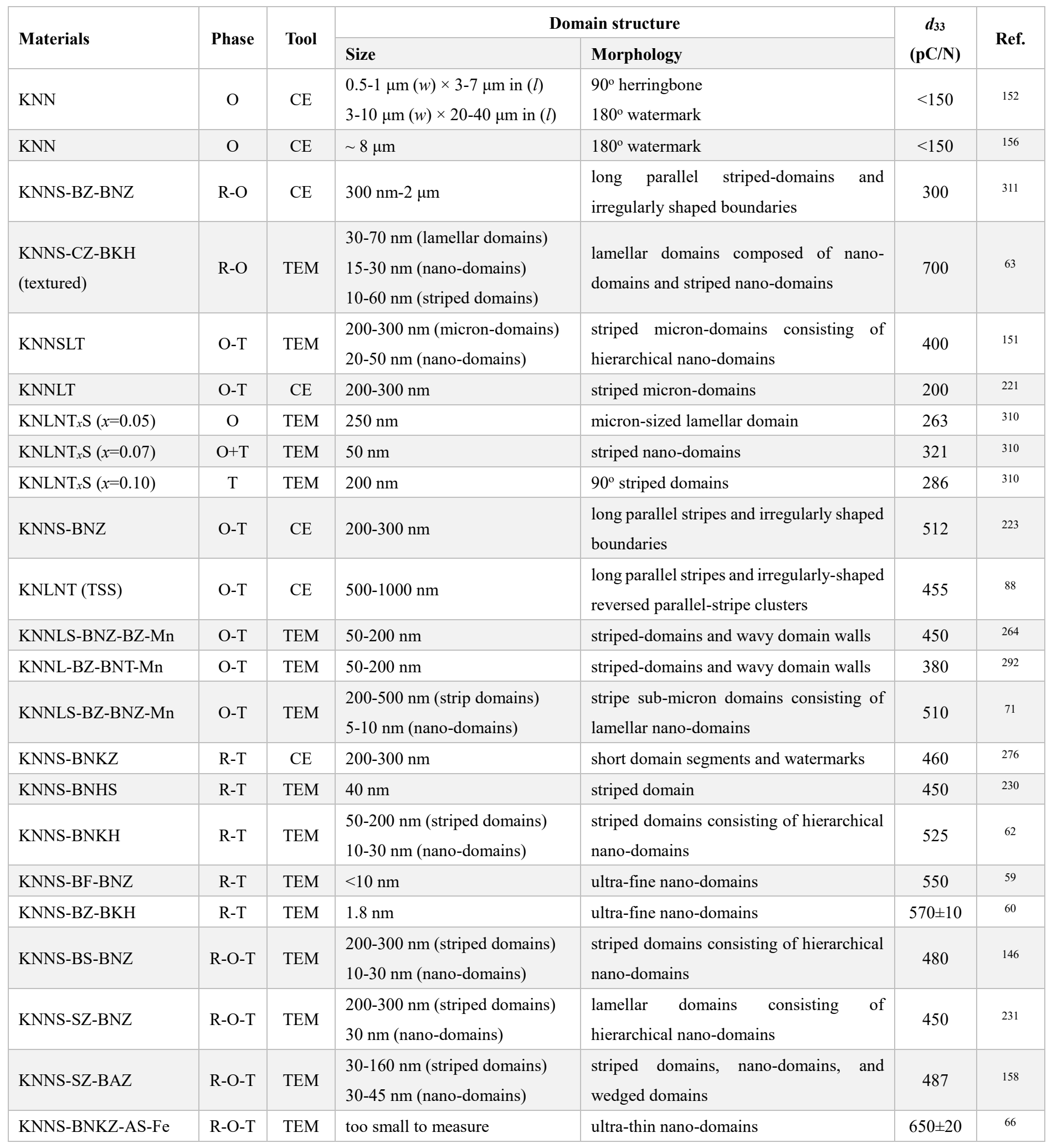

* " $w$ " and "l" represent the width and the length, respectively. 

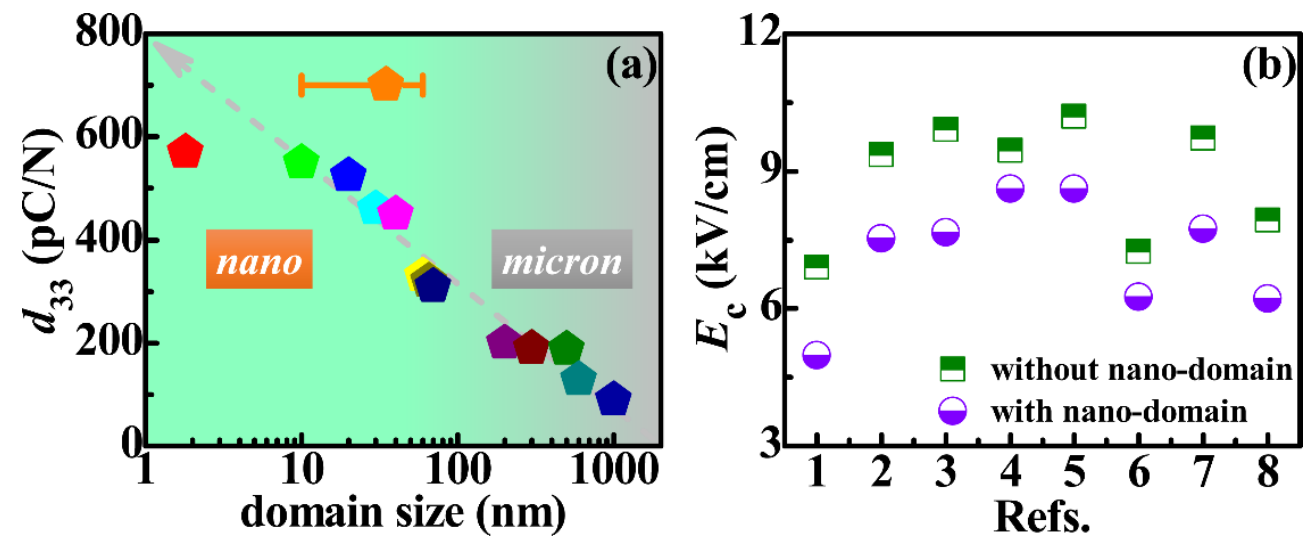

Figure 9. (a) Relationship between domain size and $d_{33}$ values in KNN-based ceramics; (b) $E_{\mathrm{c}}$ values of KNN-based ceramics with nano-domains and their counterparts without nano-domains. ${ }^{59,60,62,64,146,158,230,233}$

Inspired by the relationship between $d_{33}$ values and the domain size, our group introduced $\mathrm{AgSbO}_{3}$ and $\mathrm{Fe}_{2} \mathrm{O}_{3}$ into high-performance $0.96 \mathrm{~K}_{0.48} \mathrm{Na}_{0.52} \mathrm{Nb}_{0.95} \mathrm{Sb}_{0.05} \mathrm{O}_{3}-0.04 \mathrm{Bi}_{0.5}\left(\mathrm{Na}_{0.82} \mathrm{~K}_{0.18}\right)_{0.5} \mathrm{ZrO}_{3}$ ceramics (KNNS-BNKZ- $x$ AS-Fe), to construct a relaxor slush polar state with nanoscale multi-phase coexistence and achieve a higher $d_{33}$ value. ${ }^{66}$ Figure 10 (a) shows that the whole area of KNNS-BNKZ$x \operatorname{AS}-\mathrm{Fe}(x=1.6 \%)$ ceramic only exhibited the ultra-thin nano-domains with unmeasurable scale. Scanning transmission electron microscope high-angle annular dark field (STEM HAADF) image proved these ultra-thin nano-domains to be the coexistence of $\mathrm{R}, \mathrm{O}$, and $\mathrm{T}$ phases (see Figure 10(b)), which resulted in the strong relaxor behavior (see Figure 10(c)). Finally, an ultra-high $d_{33}$ of $650 \pm 20$ $\mathrm{pC} / \mathrm{N}$ was observed in KNNS-BNKZ- $x$ AS-Fe $(x=1.6 \%)$ ceramics, the highest value in non-textured KNN-based ceramics reported so far (see Figure 10(d)). ${ }^{66}$ Therefore, it is hopeful for achieving high piezoelectric properties by controlling the size of the nano-domain through chemical modification. 

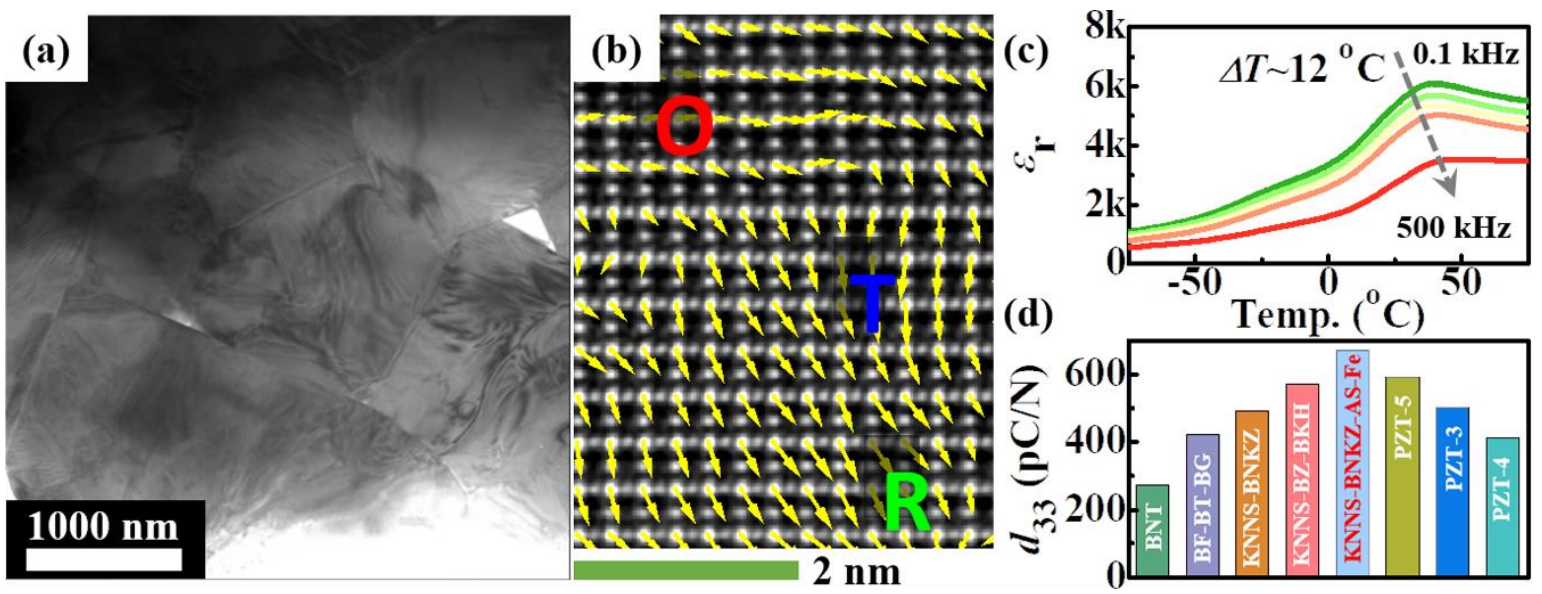

Figure 10. (a) TEM image, (b) STEM HAADF image, and (c) temperature-dependent permittivity $\left(\varepsilon_{\mathrm{r}}-T\right)$ curves of KNNS-BNKZ- $x$ AS-Fe $(x=1.6 \%)$ ceramics; (d) Comparison of $d_{33}$ values among the state-of-the-art non-textured lead-free ceramics and the representative commercial lead-based ceramics. (a-d) Reproduced from ref. 66. with permission from the American Chemical Society, Copyright 2019.

As we know, piezoelectric single crystals generally exhibit much better electromechanical properties than their ceramics because of the well-aligned polarization orientation and domain engineering. ${ }^{312-314}$ Inspired by the piezoelectricity enhancement induced by the nano-domains in KNN-based piezoceramics, Jiang et al. prepared the well-designed (1-x)(99.6 $\left.\mathrm{K}_{0.5} \mathrm{Na}_{0.5} \mathrm{NbO}_{3}-0.4 \mathrm{LiBiO}_{3}\right)-x \mathrm{MnO}_{2}$ single crystals by the using a simple seed-free solid-state growth method (see the inset in Figure 11(a)). ${ }^{86}$ Previously, it was reported that the piezoelectric properties of KNN-based single crystals with an $\mathrm{O}$ phase can be better if measured along $<001>$ zone axis. ${ }^{86,315-317}$ Therefore, they cut the KNN-LB: $0.375 \% \mathrm{MnO}_{2}$ single crystal into a pellet with the $(002)_{\mathrm{pc}}$ orientation (see Figure 11(a)). TEM image showed that the $\mathrm{KNN}-\mathrm{LB}: 0.375 \% \mathrm{MnO}_{2}$ single crystal contained abundant nano-size lamellar domains with the scale of several tens of nanometers (see Figure 11(c)), which were much smaller than those of Mn-free KNN-based single crystals. ${ }^{108,109,315-356}$ Subsequently, an extremely high $d_{33}$ value of 1050 pC/N was observed in $\mathrm{KNN}-\mathrm{LB}$ : $0.375 \% \mathrm{MnO}_{2}$ single crystal, the highest value among $\mathrm{KNN}$-based 
ceramics and single crystals reported so far (see Figure $11(\mathrm{c})) .{ }^{108,109,315-356}$ Besides, an ultra-high $d_{33}{ }^{*}$ value of $2290 \mathrm{pm} / \mathrm{V}$ was also observed (see Figure 11(d)). Therefore, the nano-domain engineering can substantially promote the piezoelectric properties of both piezoelectric single crystals and piezoceramics.
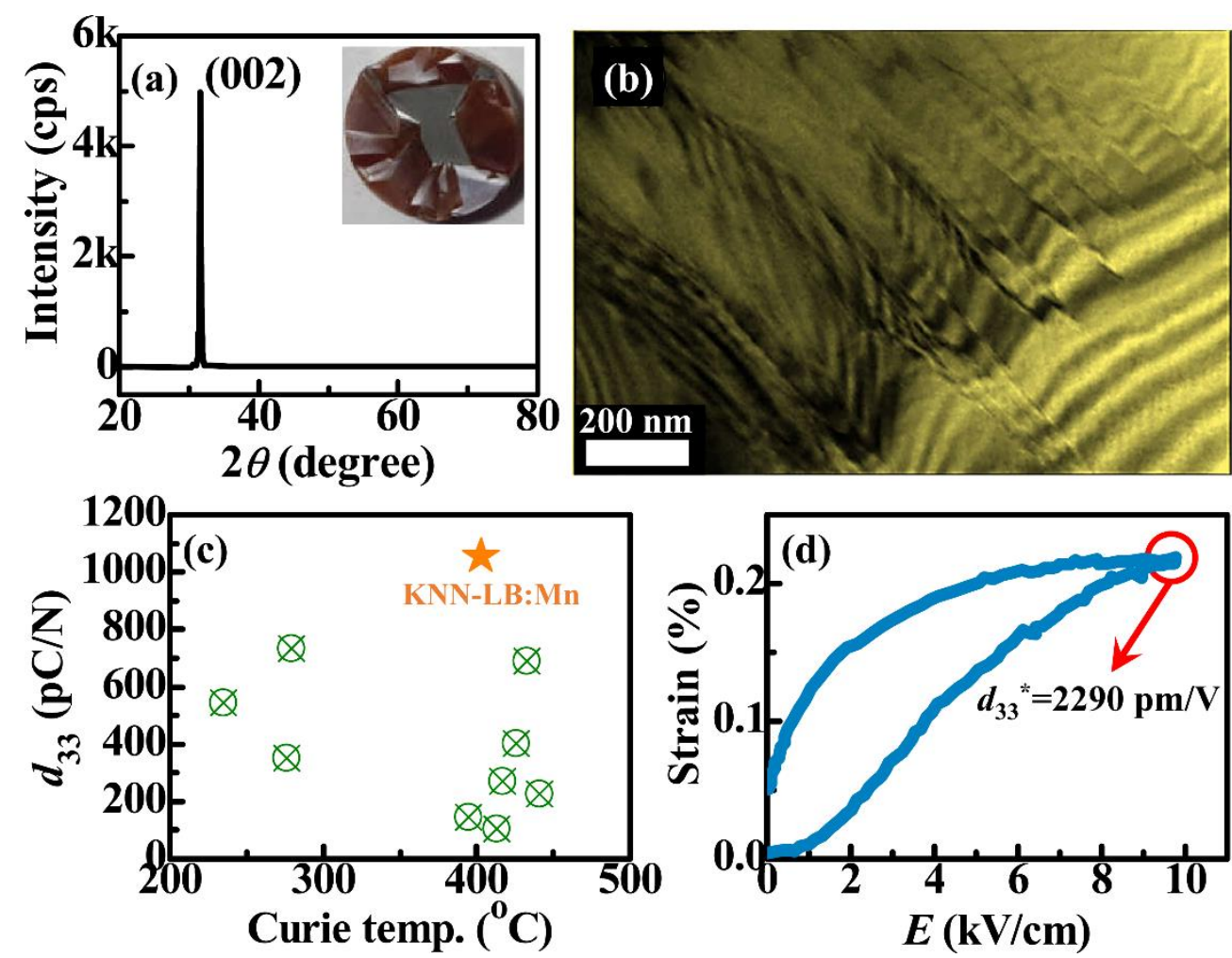

Figure 11. (a) XRD pattern, (b) ferroelectric domain, and (d) unipolar strain curve of the as-grown KNN-LB: $0.375 \% \mathrm{MnO}_{2}$ single crystal. (c) Statistics diagram of $d_{33} v s . T_{\mathrm{c}}$ among different $\mathrm{KNN}$-based single crystals. The inset of (a) shows the photo of KNN-LB: $0.375 \% \mathrm{MnO}_{2}$ single crystal. (a-d) Reproduced from ref. 86 . with permission from the Royal Society of Chemistry, Copyright 2019.

The above-discussed domains were observed from the unpoled KNN-based ceramics, which have not the net piezoelectricity because of the lack of applying an electric field that induces the domain switching and domain wall motion. Thus, it is important to observe the domain structure from both unpoled and poled KNN-based ceramics for explaining the effects of nano-domains on piezoelectric 
properties. Zhang et al. and Wu et al. observed the nano-domains of both poled and unpoled KNNbased ceramics using TEM and CE+SEM methods. ${ }^{158,223,276}$ Before poling, the nano-domains exhibited the size of 20-200 $\mathrm{nm}$ and the complicated configurations with the shapes of the striped, the short segment, and the watermark (see Figure 12(a1, a2, b1, b2)). After poling, the nano-domains were simplified. The major nano-domains exhibited the striped shape with a size of 20-100 nm (see Figure 12(a3, b3, b4)). The simplification of nano-domains is caused by the disappearance of the $180^{\circ}$ domain wall and non- $180^{\circ}$ domain switching. In particular, a single-domain structure was found in poled KNNS-SZ-BAZ ceramics (see Figure 12(a4)), which was also observed in high-performance 50BZT50BCT ceramics during in situ electric-dependent domain observation and believed to be responsible for the high piezoelectric properties. ${ }^{357-360}$

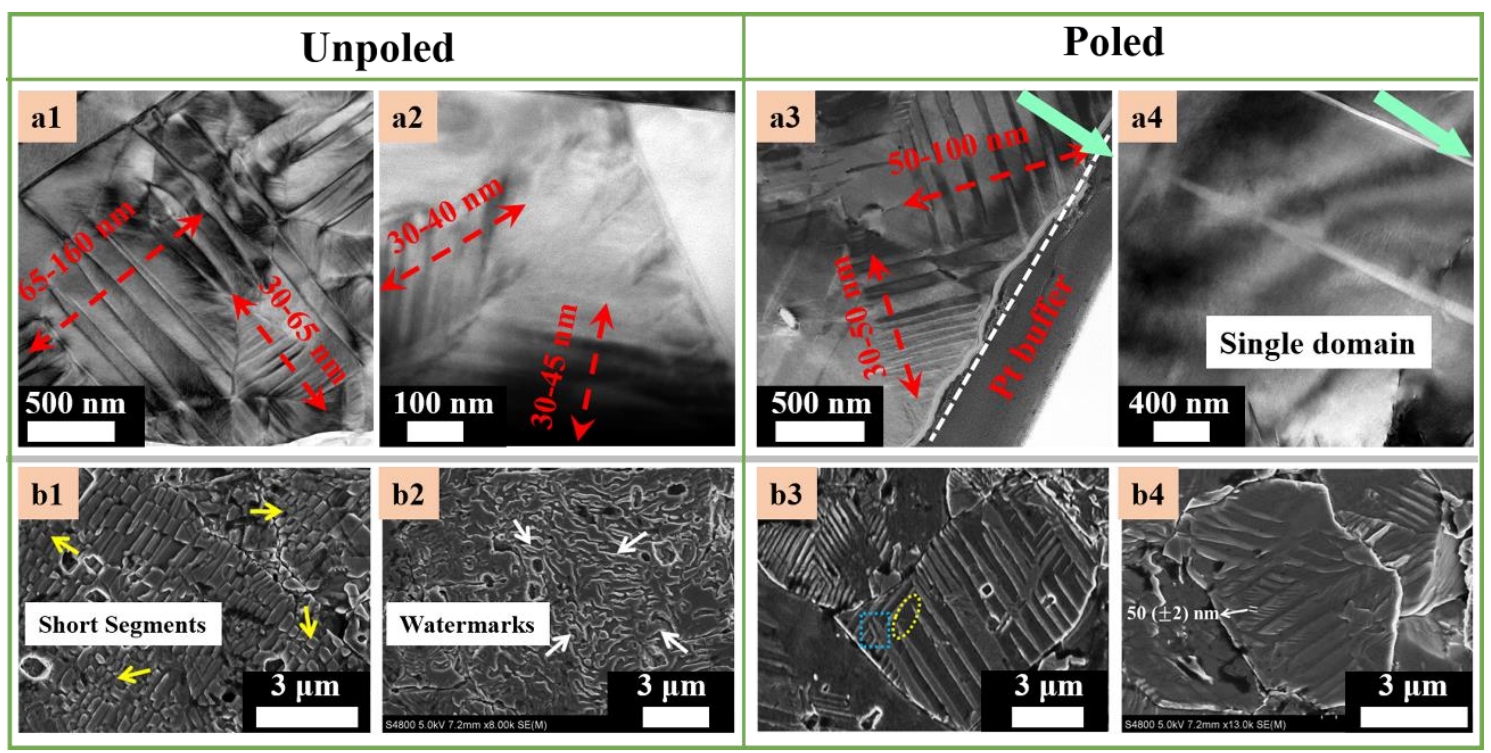

Figure 12. Nano-domains of unpoled and poled KNN-based ceramics. (a1-a4) from KNNS-SZ-BAZ ceramics, ${ }^{158}$ and (b1-b4) from KNNS-BNKZ ceramics. ${ }^{276}$ The cyan arrows in Figures 7(a3, a4) indicate the direction of the electric field. (a1-a4) Reproduced from ref. 158. with permission from the Royal Society of Chemistry, Copyright 2019; (b1b4) Reproduced from ref. 276. with permission from the American Chemical Society, Copyright 2016. 


\subsection{Nano-domain vs. Strain properties}

Strain properties of ferroelectrics are closely related to the domain switching and domain wall motion, particularly the non- $180^{\circ}$ ferroelectric domains. ${ }^{361}$ Generally, the strain of ferroelectric materials originates from the i) intrinsic converse piezoelectric effect, ii) non- $180^{\circ}$ domain switching and domain wall motion, iii) electrostriction effect, and v) possible electric-induced phase transition. ${ }^{41}$ In particular, the negative strain $\left(S_{\text {neg }}\right)$ induced by a bipolar electric field mainly arises from the non- $180^{\circ}$ domain switching and domain wall motion, which is suitable for evaluating the effect of domain size on the strain properties. ${ }^{361} \mathrm{Wu}$ et al. systematically investigated the effects of domain size on strain properties of KNN-based ceramics (see Figure 13). ${ }^{233}$ As the domain size reduced from micron-scale to nanoscale (see Figure 13(a, b, d, e)), both the positive strain $\left(S_{\text {pos }}\right)$ and $S_{\text {neg }}$ sharply increased (see Figure $13(\mathrm{~g}$, h)). As the domain size further reduced, the whole grain showed only PNRs instead of the obvious ferroelectric domains (see Figure 13(c, f)). Then, its bipolar strain curve exhibited distinct $S_{\text {pos }}$ and negligible $S_{\text {neg }}$ (see Figure 13(i)), indicating the dominating electrostriction effect. ${ }^{38}$ Therefore, the occurrence of nano-domains substantially promoted the non- $180^{\circ}$ domain switching and domain wall motion because of the easy switching and rapid response to the external stimulus (e.g., electric field and stress), resulting in the enhanced $S_{\text {neg. }}$. But pure PNRs produced a pure electrostriction strain with no hysteresis, showing the promising applications in precise control. ${ }^{38,362-364}$ This is not an exclusive phenomenon for KNNS-SZ- $x$ BNZ ceramics, but a common one for other KNN-based ceramics with PBE. With the increasing content of additives, the phase structure firstly changes from a pure O phase to a multi-phase coexistence (e.g., O-T, R-O-T, or R-T) and finally becomes a pseudo-cubic (or relaxor) phase. ${ }^{90,92,233,266,276,278-284}$ The corresponding domain structure undergoes the evolution like that of KNNS-SZ- $x$ BNZ ceramics. Here we collected the $S_{\text {neg }}$ of PBE-modified KNN-based ceramics where 
the different domain sizes are reasonably anticipated (see Figure 14). ${ }^{90,92,233,266,276,278-284}$ KNN-based ceramics with nano-domains exhibited the much higher $S_{\text {neg }}$ than those of the ones with microndomains, while KNN-based ceramics comprised of PNRs display the negligible $S_{\text {neg. }}$.
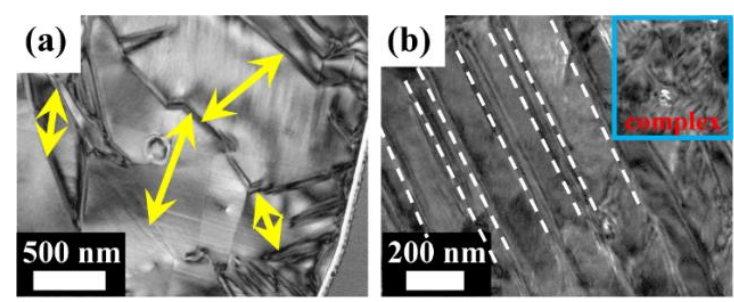

(d)
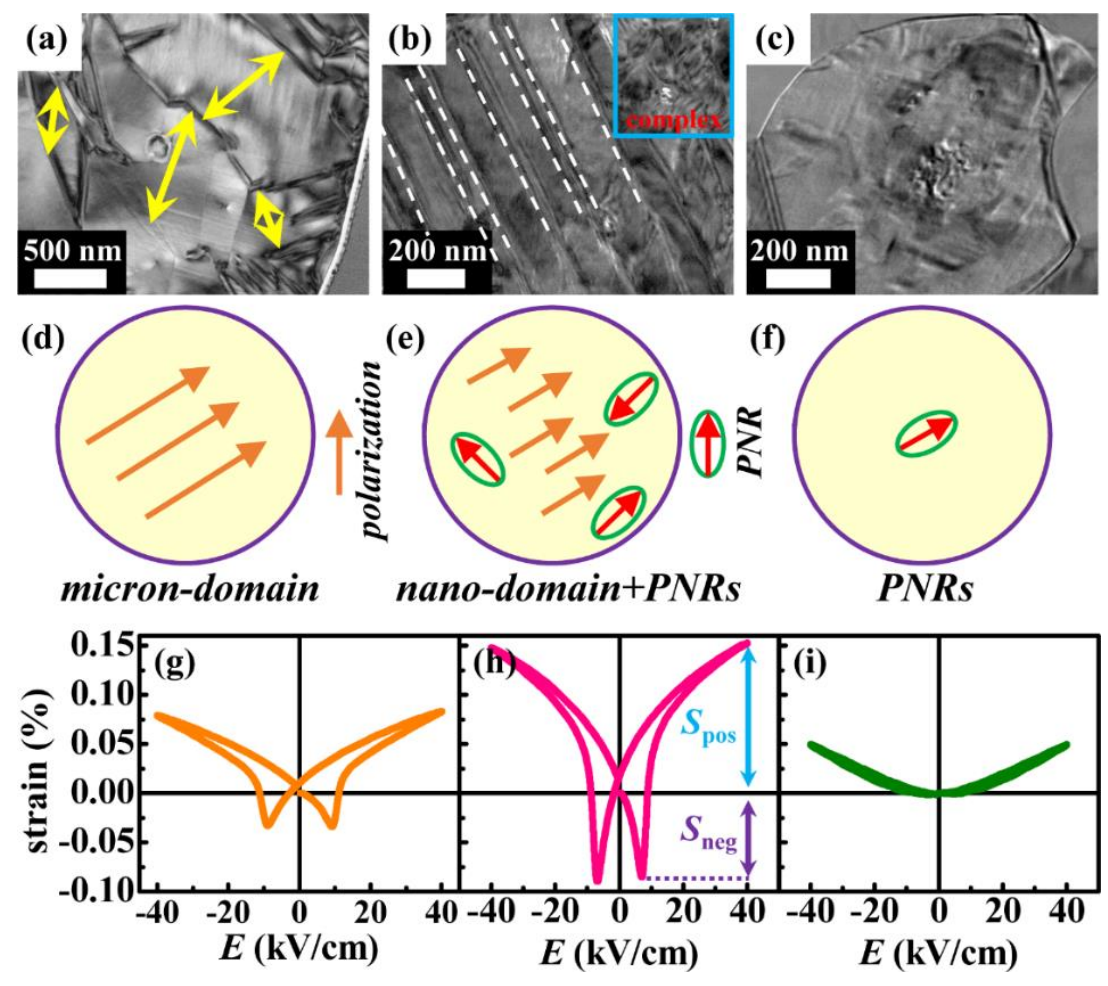

Figure 13. Domain structure, schematic diagrams of domains, and bipolar strain curves of KNN-SZ-xBNZ ceramics with (a, d, g) $x=0,(\mathrm{~b}, \mathrm{e}, \mathrm{h}) x=0.03$, and (c, f, i) $x=0.05 .{ }^{233}$ (a-i) Reproduced from ref. 233. with permission from the Royal Society of Chemistry, Copyright 2019.

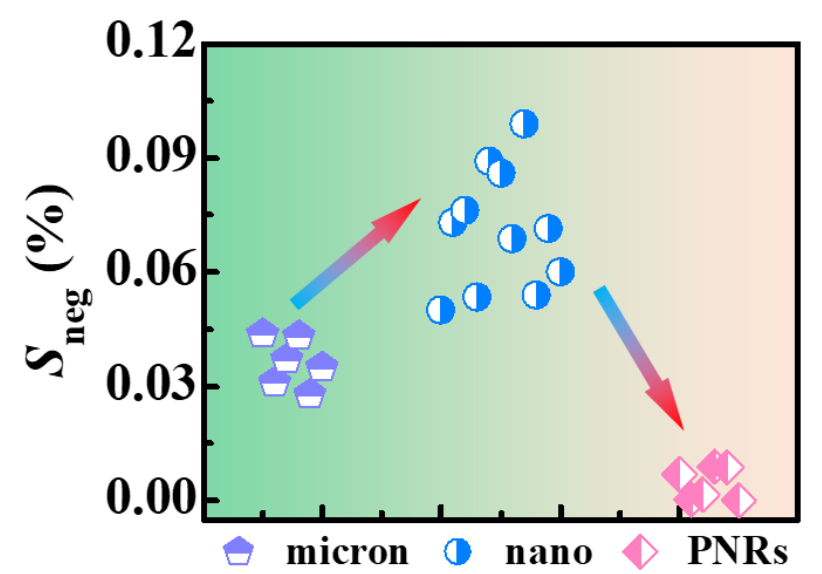

Figure 14. $S_{\text {neg }}$ of PBE-modified KNN-based ceramics with different domain sizes. ${ }^{90,92,233,266,276,278-284}$ 
Except for the composition-induced variation of domain size, the grain size also affects the domain size according to Equation (1). Thus, Zhai et al. used the combined methods to control the grain size of $95 \mathrm{KNNS}-1 \mathrm{CZ}-4 \mathrm{BKHT}$ ceramics and measured their bipolar strain curves (see Figure 15(a)). ${ }^{277}$ With the increasing grain size, $S_{\text {neg }}$ rapidly increased at a grain size of $0.31-2.55 \mu \mathrm{m}$, then slowly increased at a grain size of 2.84-3.55 $\mu \mathrm{m}$, and finally slightly reduced at a grain size of 3.55-3.83 $\mu \mathrm{m}$ (see Figure 15(a,b)). This phenomenon was well explained by the variations of domain size. As the grain size increased from 0.31 to $2.55 \mu \mathrm{m}$, the domain size changed from the PNRs to the nanoscale,

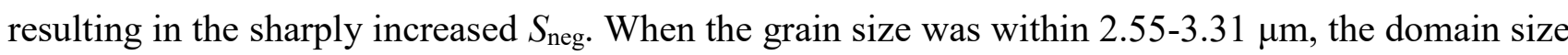
slightly fluctuated, leading to the slightly increased $S_{\text {neg. }}$. As the grain size exceeded $3.31 \mu \mathrm{m}$, the domain size may gradually transform from the nanoscale to the micron-scale, generating the slightly decreased $S_{\text {neg. }}$ This conclusion was then demonstrated by TEM images (see Figure 15(c, d)). Only a small amount of featureless domains (marked by the white arrow) were observed in the ceramics with a grain size of $0.76 \mu \mathrm{m}$ (see Figure 15(c)), and abundant striped nano-domains with a scale of $60-80$ $\mathrm{nm}$ (marked by the yellow arrow) were found in the ceramics with a grain size of $3.31 \mu \mathrm{m}$ (see Figure 15(d)). Considering the same phase structure of 95KNNS-1CZ-4BKHT ceramics at a grain size range of 1.04-3.83 $\mu \mathrm{m}$, the nano-domains indeed enhanced the strain properties of KNN-based ceramics. Here, it is should be pointed out that the reduced residual stress in the coarse grains also contributes to the easy domain switching. ${ }^{130,167}$ Therefore, the nano-domains affect strain properties by cooperating with other factors, such as residual stress. 

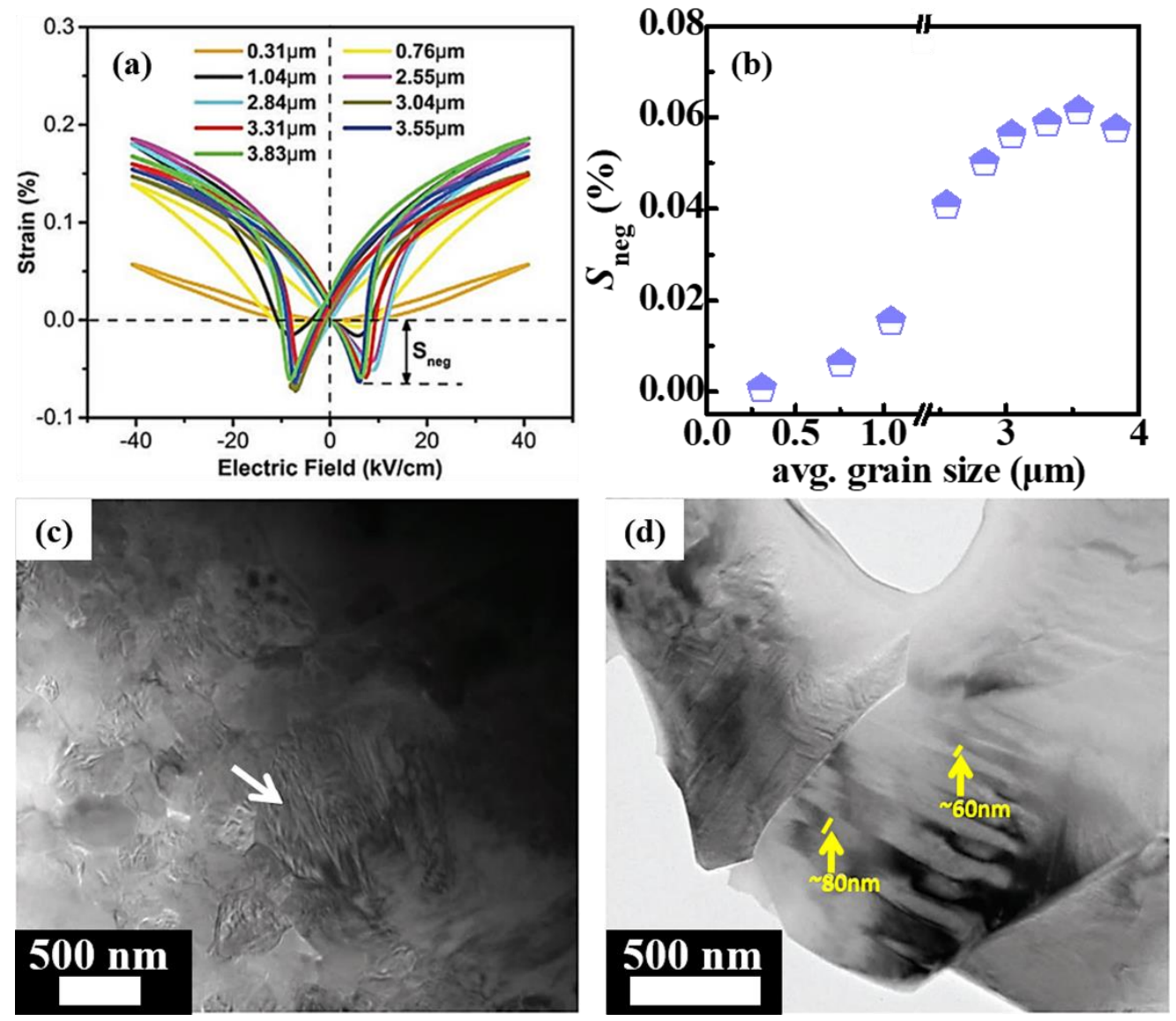

Figure 15. (a) Bipolar strain curves and (b) $S_{\text {neg }}$ of $0.95\left(\mathrm{~K}_{0.5} \mathrm{Na}_{0.5}\right)\left(\mathrm{Nb}_{0.965} \mathrm{Sb}_{0.035}\right) \mathrm{O}_{3}-0.01 \mathrm{CaZrO}_{3}-$ 0.04 $\left(\mathrm{Bi}_{0.5} \mathrm{~K}_{0.5}\right)\left(\mathrm{Hf}_{0.98} \mathrm{Ti}_{0.02}\right) \mathrm{O}_{3}(95 \mathrm{KNNS}-1 \mathrm{CZ}-4 \mathrm{BKHT})$ ceramics with different grain sizes. Domain structure of 95KNNS-1CZ-4BKHT ceramics with a grain size of (c) $0.76 \mu \mathrm{m}$ and (d) $3.31 \mu \mathrm{m}$. (a-d) Reproduced from ref. 277. with permission from the Wiley-VCH, Copyright 2019.

Nano-domains not only work in the strain enhancement of normal ferroelectric materials, but also of ferroelectric materials with a core-shell structure. ${ }^{365}$ Choi et al. prepared the KNLNT-CZ ceramic that exhibited a nanoscale core-shell structure (see Figure 16(a)). ${ }^{365}$ STEM atomic-scale annular brightfield (STEM ABF) images from the poled KNLNT-CZ ceramics showed that the lattice of the core region can be effectively changed, and the lattice of shell region almost remained unchanged (see Figure 16(b, c)). Therefore, the core region was in a ferroelectric polar state, whereas the shell was in 
a paraelectric non-polar state. Then, they observed a unipolar strain $\left(S_{\text {uni }}\right)$ as high as $0.4 \%$ in KNLNT$\mathrm{CZ}$ ceramics, higher than those of other representative KNN-based ceramics and that of soft commercial PZT ceramics (see Figure 16(d)). By comparing the polarization reorientation of normal ferroelectric ceramics and KNLNT-CZ ceramics during the measurement, they attributed the enhanced strain to the non-polar state of shell region that easily accepted the polarization extension from core region under an electric field and turned back to the initial state after removing the electric field.
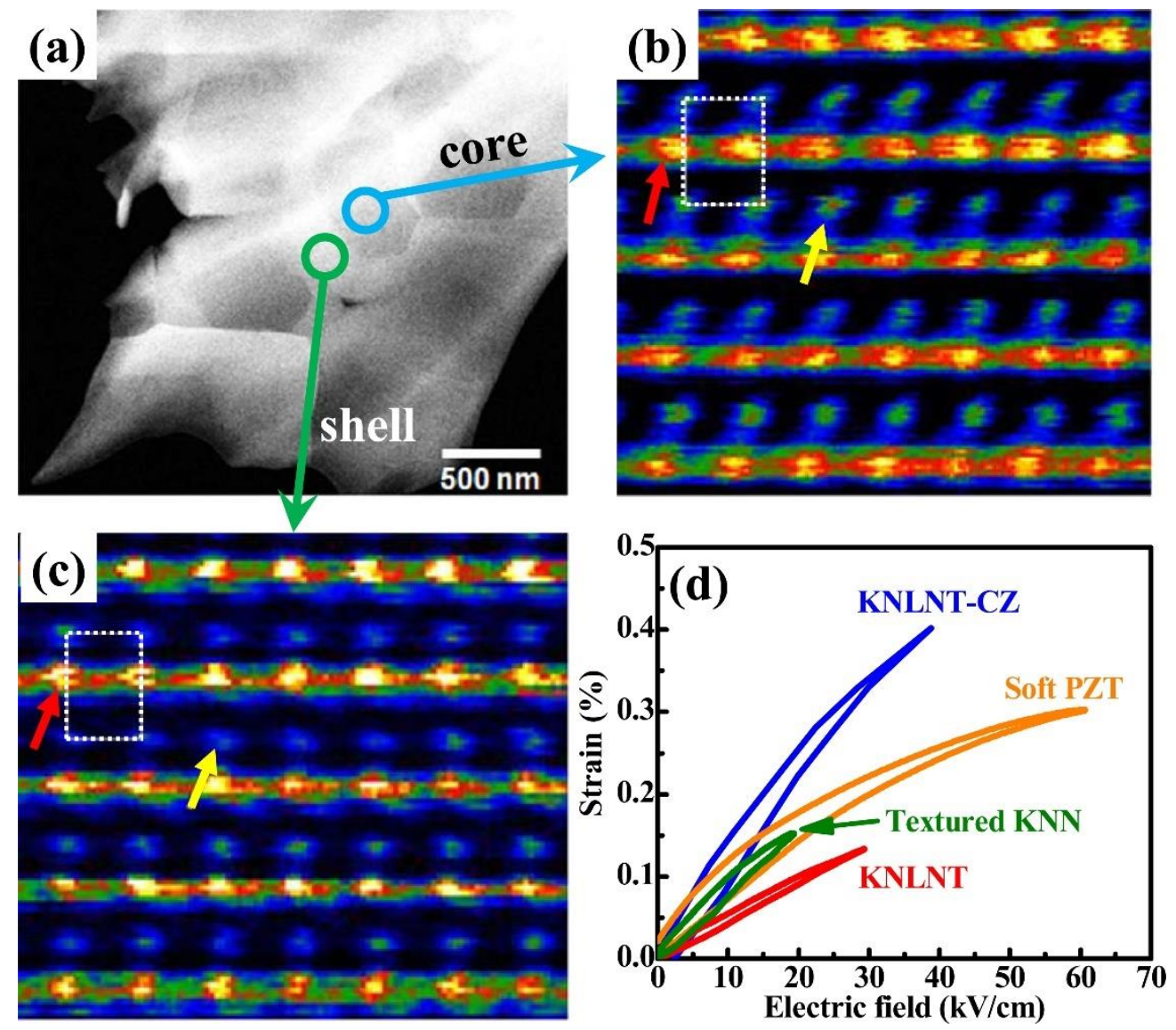

Figure 16. (a) STEM HAADF image of the core-shell structure of $0.96\left(\mathrm{~K}_{0.51} \mathrm{Na}_{0.47} \mathrm{Li}_{0.02}\right)\left(\mathrm{Nb}_{0.8} \mathrm{Ta}_{0.2}\right) \mathrm{O}_{3}-0.04 \mathrm{CaZrO}_{3}$ (KNLNT-CZ) ceramics. STEM ABF images from the (b) core and (c) shell regions after poling. (d) Unipolar strain curves of commercial soft PZT ceramics and several representative KNN-based ceramics. The red arrow indicates the location of B-site atom $(\mathrm{Nb} / \mathrm{Ta})$, while the yellow arrow shows the location of A-site atom $(\mathrm{K} / \mathrm{Na} / \mathrm{Li}) .{ }^{365}(\mathrm{a}-\mathrm{d})$ Reproduced from ref. 365. with permission from the American Chemical Society, Copyright 2012. 


\subsection{PNRs vs. Piezoelectricity}

PNRs, with a scale of 1-10 nm, play an important role in piezoelectric properties and are believed to be responsible for the relaxor phenomenon and the high piezoelectricity in PMN-PT and PZN-PT material systems. ${ }^{79,80,82,147,187,188,202}$ However, the direct observations for PNRs were rarely reported because of the difficulty in finding them. Recently, Li et al. and Wu et al. used high-resolution TEM (HR-TEM) to observe the PNRs in KNN-based ceramics (see Figure $17(\mathrm{a}-\mathrm{c})$ ). ${ }^{64,158}$ PNRs were found to be embedded in the long-range ordered ferroelectric domain matrix and exhibited the scale of 1-5 nm (see Figure 17(a-c)). Generally, PNRs are believed to act as an "accelerator" or a "seed" during the domain switching, resulting in the easy domain switching and benefiting the net piezoelectric properties. $79,80,82,147,187,188,202$ This conclusion is widely recognized in both lead-free and lead-based piezoelectric materials. ${ }^{64,79,80,82,147,158,187,188,202,366}$ Here we'd like to emphasize the double-edged role of PNRs in lead-free piezoceramics. Generally, the relaxor degree is evaluated via a modified Curie-Weiss law.

$$
\frac{1}{\varepsilon(T)}-\frac{1}{\varepsilon_{m}}=\frac{\left(T-T_{m}\right)^{\gamma}}{C}
$$

where $\varepsilon_{\mathrm{m}}$ represents the maximum permittivity at $T_{\mathrm{m}}, C$ is the Curie-like constant, and $\gamma$ is the diffusion factor. ${ }^{147}$ Classical ferroelectrics possess a $\gamma$ of 1 , while the ideal relaxor one owns a $\gamma$ of $2 .{ }^{147}$ As shown in Figure 17(d, e), the application of PBE increased the relaxor degree because of the increasing content of additives, that is, $\gamma$ increased from 1.32 (for $x=0$ ) to 1.57 (for $x=0.035$ ). ${ }^{158}$ Considering the easy domain switching caused by the multi-phase coexistence and PNRs, it was highly anticipated that KNNS-SZ- $x$ BAZ $(x=0.035)$ ceramics shall achieve a higher poling saturation than KNNS-SZ- $x$ BAZ $(x=0)$ ceramics under the electric field. Surprisingly, the experimental results showed a contrary 
phenomenon. After poling, $\gamma$ of KNNS-SZ- $x$ BAZ $(x=0)$ ceramics reduced from 1.32 to 1.19 (see Figure $17(\mathrm{~d})$ ), and KNNS-SZ- $x$ BAZ $(x=0.035)$ ceramics exhibited an almost unchanged $\gamma$ of 1.52 (see Figure 17(e)), indicating the still existence of substantial PNRs. The corresponding phase angle $(\theta)$ after poling also supported this, that is, KNNS-SZ- $x$ BAZ $(x=0)$ ceramics exhibited a $\theta$ of $74^{\circ}$, but a much lower $\theta$ of $58^{\circ}$ was observed in KNNS-SZ- $x$ BAZ $(x=0.035)$ ceramics (see Figure $\left.17(\mathrm{f})\right)$. One the one hand, PNRs of lead-free piezoceramics can promote the domain switching because of the easy response to the electric field; on the other hand, it is difficult to completely re-orientate the PNRs along with the electric field. ${ }^{147,158}$ The high content of PNRs is associated with indispensable additives. ${ }^{158}$ Therefore, PNRs of lead-free piezoceramics are the double-edged "sword".
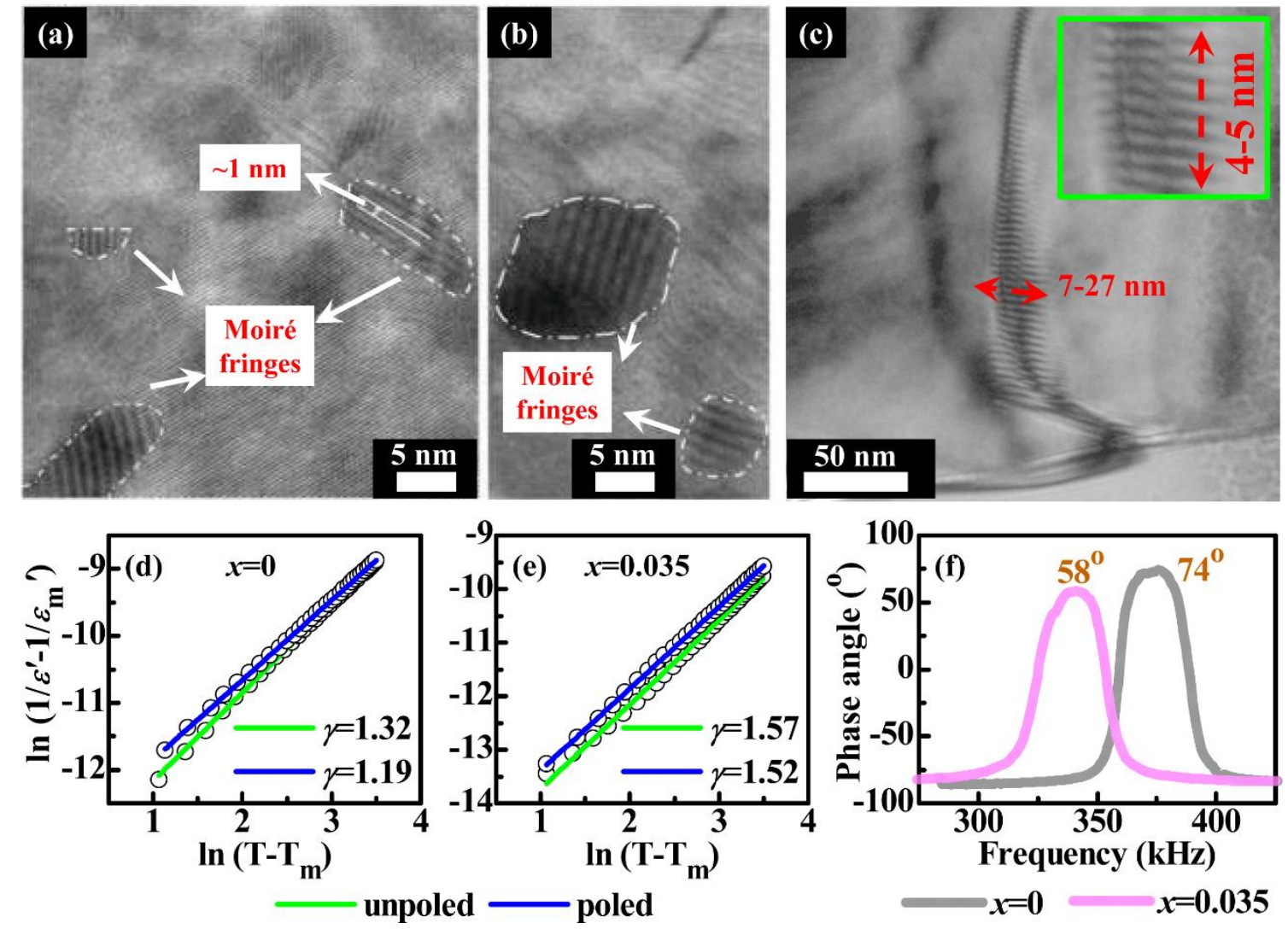

Figure 17. TEM images of PNRs of $(\mathrm{a}, \mathrm{b}) \mathrm{KNNS}_{x}$-BZ-BNH-MnO $2(x=0.025)$ ceramics and (c) KNNS-SZ- $x \mathrm{BAZ}$ $(x=0.035)$ ceramics. $^{64,158}(\mathrm{~d}, \mathrm{e})$ Linear fitting of $\ln \left(\mathrm{T}-\mathrm{T}_{\mathrm{m}}\right)$ and $\ln \left(1 / \varepsilon^{\prime}-1 / \varepsilon_{m}^{\prime}\right)$, as well as (f) phase angle of KNNS-SZ-

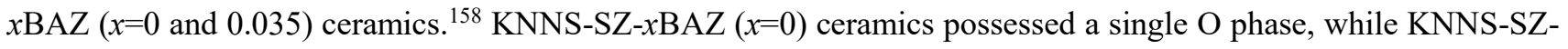


$x$ BAZ $(x=0.035)$ ceramics exhibited a diffused R-O-T multi-phase coexistence. $(\mathrm{a}, \mathrm{b})$ Reproduced from ref. 64. with permission from the Royal Society of Chemistry, Copyright 2018; (c-f) Reproduced from ref. 158. with permission from the Royal Society of Chemistry, Copyright 2019.

\subsection{Nano-domain vs. Temperature stability}

Temperature stability is an important factor for practical applications, which is one of the biggest challenges for lead-free ceramics. ${ }^{6,9,12,14,15,41,44}$ As we know, the intrinsic trait of polymorphic phase boundary (PPB) is to show a distinct temperature-dependent piezoelectric response. ${ }^{6,9,12,14,15,41,44}$ Ferroelectric domains, acting as the extrinsic contribution, are also responsible for the temperature dependence of macro piezoelectric properties. In particular, Wu et al. and Li et al. found that nanodomains directly affected the temperature stability of KNN-based ceramics. ${ }^{62,}$ 64, 231, 235, 239, 262, 264, 266, 285-287, 292 To study the temperature stability of KNN-CZ5 ceramics, Li et al. carried out the in situ PFM measurement in the temperature range of $25-150{ }^{\circ} \mathrm{C}$ (see Figure $18(\mathrm{a}-\mathrm{d})$ ). ${ }^{287}$ At room temperature, KNN-CZ5 ceramics exhibited considerable nano-domains (see Figure 18(a)). With increasing temperature, nano-domains gradually shrunk (see Figure 18(a-d)). The histograms and the line scan profiles further demonstrated the gradual depolarization process (see Figure 18(e-h)). Therefore, they attributed the massive loss of small signal piezoelectric properties to the severely depressed extrinsic contribution from domain wall motions and intrinsic counterpart from lattice displacement. ${ }^{287}$ 

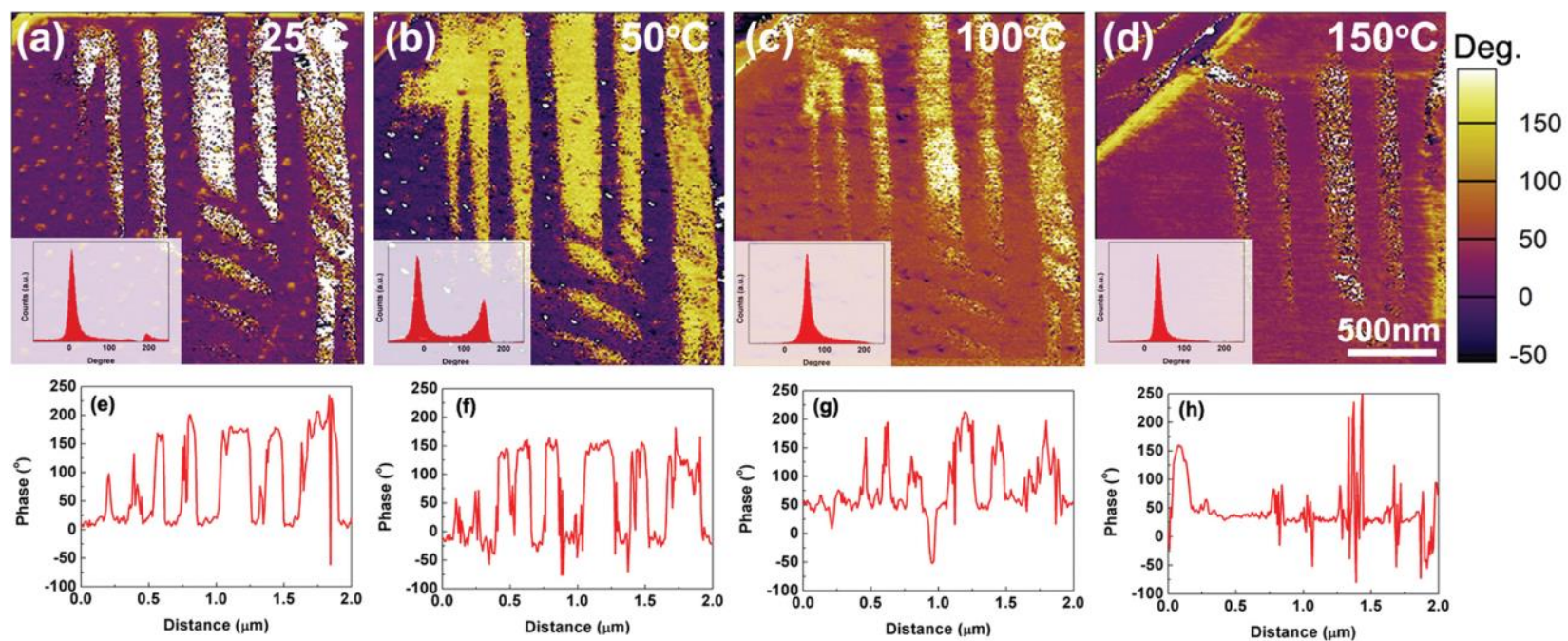

Figure 18. (a-d) Piezo-response images of unpoled $0.95\left(\mathrm{~K}_{0.49} \mathrm{Na}_{0.49} \mathrm{Li}_{0.02}\right)\left(\mathrm{Nb}_{0.8} \mathrm{Ta}_{0.2}\right) \mathrm{O}_{3}-0.05 \mathrm{CaZrO}{ }_{3}(\mathrm{KNN}-\mathrm{CZ} 5)$ ceramics measured at $25-150{ }^{\circ} \mathrm{C}$. The inset on the left bottom shows the phase histogram, in which the peaks demonstrate the distribution of domains with distinct orientations. (e-h) Corresponding piezo-response phase profiles generated from the line scan across the nano-domains. ${ }^{287}$ (a-h) Reproduced from ref. 287. with permission from the Wiley-VCH, Copyright 2016.

Recently, the PBE proposed by $\mathrm{Wu}$ et al. was widely reported to simultaneously improve the piezoelectric properties and relieve the temperature dependence. ${ }^{62,93,157,231,233,235,236,239,266,285,290,296}$ To understand the enhanced temperature stability, Wu et al. compared the temperature stability of ferroelectric domains among KNN-based ceramics with different phase structures. ${ }^{262}$ Figure 19 (a-c) shows the variations of nano-domains in $\mathrm{KNN}$-based ceramics with different phase structures and temperatures. ${ }^{262}$ With increasing temperature, nano-domains of KNN-based ceramics with an R-T phase coexistence gradually reduced in amplitude and content, but the ones of KNN-based ceramics with an R-O or O-T phase coexistence rapidly faded (see Figure 19(a-c)). Therefore, the stability of nano-domains was $\mathrm{R}-\mathrm{T}>\mathrm{R}-\mathrm{O} \approx \mathrm{O}-\mathrm{T}$. Then, variations of corresponding piezoelectric properties (e.g., $d_{33}{ }^{*}$ and $d_{33}{ }^{*} \mathrm{~T} / d_{33}{ }^{*}$ RT values) with temperature were also collected (see Figure 19(d, e)). KNN-based 
ceramics with an R-T phase coexistence showed the slowest reduction in $d_{33}{ }^{*}$ values compared to KNN-based ceramics with an R-O or O-T phase coexistence, indicating better temperature stability. Therefore, it can be inferred that the temperature stability of KNN-based ceramics is closely related to the stability of nano-domains varying with temperature. ${ }^{262}$



Figure 19. Variations of nano-domains in KNN-based ceramics with (a) an R-T phase coexistence, (b) an O-T phase coexistence, and (c) an R-O phase coexistence with increasing temperature. (d) Temperature-dependent $d_{33}{ }^{*}$ and (e) $d_{33}{ }^{*}{ }_{\mathrm{T}} / d_{33}{ }^{*} \mathrm{RT}$ values of KNN-based ceramics with different phase structures. ${ }^{262}$ (a-e) Reproduced from ref. 262 . with permission from the Royal Society of Chemistry, Copyright 2018. 


\section{Nano-domain in BT-based ceramics}

$\mathrm{BaTiO}_{3}$ ceramic was discovered in 1941 during the second world war and was the first polycrystalline ceramic material ever discovered that exhibited ferroelectricity. ${ }^{41}$ Due to the moderate piezoelectric properties and the poor stability, BT-based ceramics were replaced by the later discovered PZT-based ceramics. ${ }^{41}$ It was not until 2009 that Ren et al. reported a high $d_{33}$ of $620 \mathrm{pC} / \mathrm{N}$ in $50 \mathrm{Ba}\left(\mathrm{Ti}_{0.8} \mathrm{Zr}_{0.2}\right) \mathrm{O}_{3^{-}}$ $50\left(\mathrm{Ba}_{0.7} \mathrm{Ca}_{0.3}\right) \mathrm{TiO}_{3}(50 \mathrm{BZT}-50 \mathrm{BCT})$ ceramics that possessed a tricritical triple point consisting of a cubic paraelectric phase (C), ferroelectric rhombohedral (R), and tetragonal (T) phases. ${ }^{54}$ Then, they further obtained a higher $d_{33}$ of $697 \mathrm{pC} / \mathrm{N}$ in $0.89 \mathrm{BaTiO}_{3}-0.11 \mathrm{BaSnO}_{3}$ ceramics that exhibited a quasiquadruple point including $\mathrm{R}, \mathrm{O}, \mathrm{T}$, and $\mathrm{C}$ phases. ${ }^{70}$ Recently, Wu et al. proposed a multi-phase convergence with broad structural flexibility, by which they obtained an ultra-high $d_{33}$ of $700 \pm 30 \mathrm{pC} / \mathrm{N}$ and a high $d_{33}$ of $>600 \mathrm{pC} / \mathrm{N}$ over a wide composition range in BTS- $x \mathrm{BCT}$ ceramics. ${ }^{65}$ Though the low $T_{\mathrm{c}}$ and poor stability, these high $d_{33}$ values strongly suggest BT-based ceramics to be a good example of achieving high piezoelectric properties in lead-free piezoceramics. ${ }^{42}$ The subsequent analysis indicates that both phase structure and domain configuration are responsible for the enhanced piezoelectric properties. ${ }^{305,360,367-386}$ In this section, the effects of nano-domains on BT-based ceramics are thus summarized.

\subsection{Nano-domain vs. Piezoelectricity}

Due to the feasibility of controlling the grain size, the effects of grain size on BT-based ceramics were largely studied. ${ }^{95,134,136,144,155,161,162,167,168,217,314,387-439}$ According to Equation (1), the effects of domain size on BT-based ceramics are realized by changing the grain size of BT-based ceramics. Previously, the study lasting more than 40 years demonstrated that the grain size plays an important 
role in the electrical properties of $\mathrm{BaTiO}_{3}$ ceramics. ${ }^{95,134,136,144,155,161,162,168,217,314,387-437}$ Figure 20 (ad) shows the representative domain structure of $\mathrm{BaTiO}_{3}$ ceramics with different average grain sizes (AGS). ${ }^{162} \mathrm{BaTiO}_{3}$ ceramics exhibited the striped domains, herringbone domains ( $90^{\circ}$ domains), and watermarked domains ( $180^{\circ}$ domains), which respectively were indicated by the letters of " $\mathrm{S}$ ", "H", and "W" in Figure 20(b-d). ${ }^{162}$ Theses domain structures were well consistent with the permitted domain structures of the $\mathrm{T}$ phase (see Figure 5(b)). More importantly, it was found that the domain size decreased with a decreasing average grain size (AGS), which coincided with Equation (1). We then collected the effects of AGS on the domain size, permittivity $\left(\varepsilon_{\mathrm{r}}\right)$, and $d_{33}$ values (see Figure 20(eg)). ${ }^{95,134,136,144,155,161,162,167,168,217,314,387-438,440}$ As expected, the larger grain size is, the larger domain size is. $\varepsilon_{\mathrm{r}}$ and $d_{33}$ increased as AGS increased within 0.1-1 (or 2) $\mu \mathrm{m}$ and reduced when AGS exceeded $2 \mu \mathrm{m}$, obtaining the maximum value of $4000-8000$ and $300-519 \mathrm{pC} / \mathrm{N}$ at an AGS of $1-2 \mu \mathrm{m}$. The maximum $d_{33}$ values at an optimized AGS was 2.7 times than that of ones with a regular AGS. ${ }^{423}$

Generally, piezoelectric properties of perovskite ferroelectrics can be expressed as

$$
d_{33}=2 Q \varepsilon P_{\mathrm{s}}
$$

where $Q$ is the electrostrictive coefficient, $\varepsilon$ is the permittivity, and $P_{\mathrm{s}}$ is the spontaneous polarization. ${ }^{39}$ $Q$ is closely related to the degree of order in the cation arrangement, which has a small effect in pure $\mathrm{BaTiO}_{3}$ ceramics with different grain sizes due to the single $\mathrm{T}$ phase. ${ }^{364} P_{\mathrm{s}}$ increased with increasing grain size, and $\varepsilon_{\mathrm{r}}$ reached the maximum at an AGS of 1-2 $\mu \mathrm{m}$, which explained why the maximum $d_{33}$ values were obtained at this AGS. To understand the effects of grain size on $\varepsilon_{\mathrm{r}}$ of $\mathrm{BaTiO}_{3}$ ceramics, the

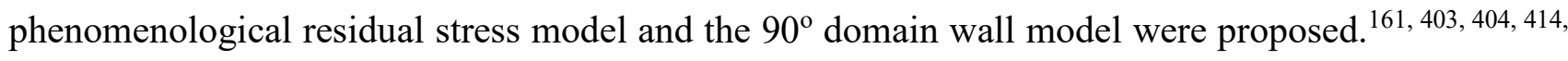
441-444 The phenomenological residual stress model proposed by Buessem et al. ascribed the observed 
maximum $\varepsilon_{\mathrm{r}}$ at an AGS of 1-2 $\mu \mathrm{m}$ to an increased internal residual stress, which was induced during the paraelectric-ferroelectric phase transition and relieved to some extent due to formation of the ferroelectric/ferroelastic tetragonal domains within every single grain of $\mathrm{BaTiO}_{3}$ ceramics at room temperature. ${ }^{44-444}$ At an AGS of 1-2 $\mu \mathrm{m}$, Buessem et al. believed that the $90^{\circ}$ domain walls did not form, indicating the internal residual stress was not relieved like the situation within coarse grains. ${ }^{441-}$ ${ }^{444}$ Then, the residual stress suppressed the tetragonality of the T phase, generating a phase structure close to the cubic phase. ${ }^{441-444}$ Generally, $\varepsilon_{\mathrm{r}}$ reached the maximum near $T_{\mathrm{c}}$, to which they ascribed the enhanced $\varepsilon_{\mathrm{r}}$. The $90^{\circ}$ domain walls model was proposed by Arlt et al. In the beginning, they attributed to the superior $\varepsilon_{\mathrm{r}}$ to the high density of $90^{\circ}$ domain walls. ${ }^{403,404,414}$ After calculating a domain width dependence of the force constant, they then ascribed it to originate from the strong softening of force constant because of the decrease of stresses in and near the grain boundary areas. To testify the validity of two models, Ghosh et al. carried out the in situ high-energy XRD measurements under the low and high electric fields. ${ }^{161}$ For $\mathrm{BaTiO}_{3}$ ceramics with different grain sizes, the composition-dependent highenergy XRD patterns before applying an electric field showed the negligible shifting of lattice, indicating the almost unchanged macro strain over the entire grain size range of $0.2-3.5 \mu \mathrm{m}$. Then, XRD patterns during the application of an electric field exhibited the obvious $90^{\circ}$ domain wall displacement, particularly in the ones with an AVG of $\sim 2 \mu \mathrm{m}$. Therefore, they mainly attributed the enhanced dielectric and piezoelectric properties in $\mathrm{BaTiO}_{3}$ ceramics at an intermediate grain size (1-2 $\mu \mathrm{m})$ to the $90^{\circ}$ domain wall displacement. ${ }^{161}$ 

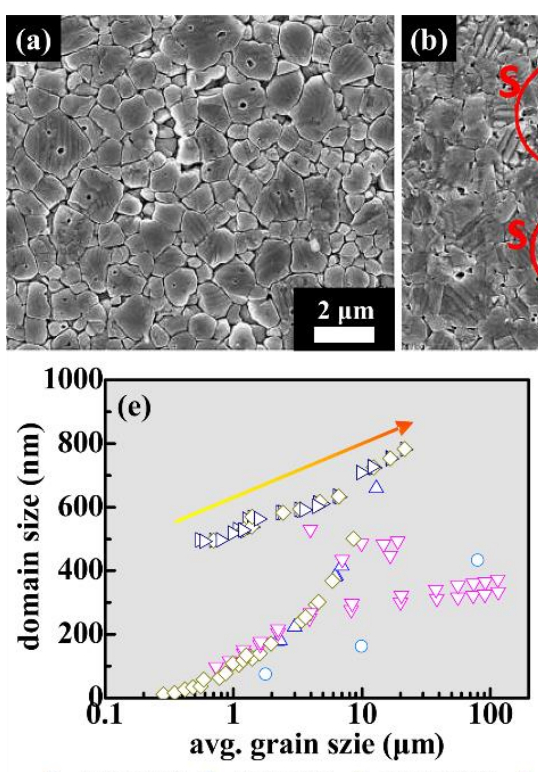

Nano+MS $\triangle$ Nano+CS $\diamond$ Nano+TSS
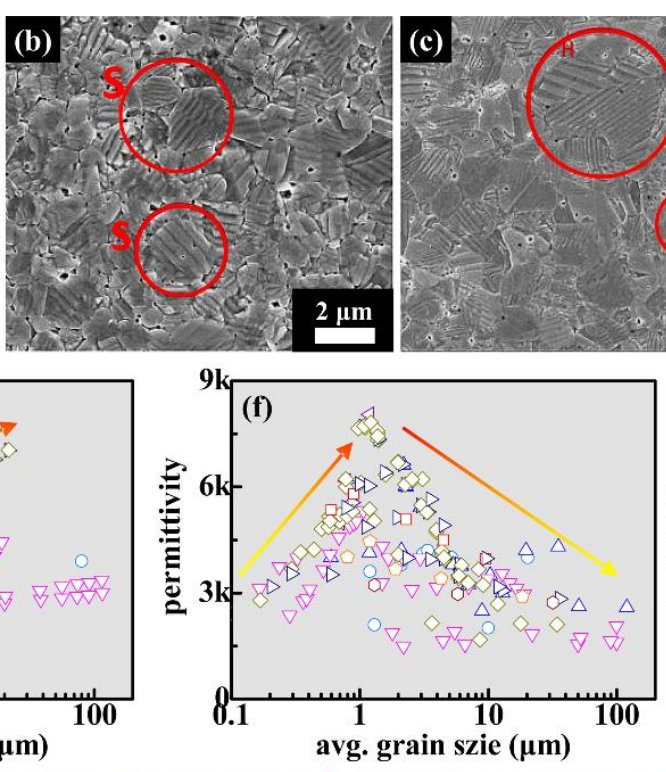

Nano+RC-TSS $\square$ Nano+SPS $\triangleright$ Nano+Mixed $\bigcirc$ Micro+CS
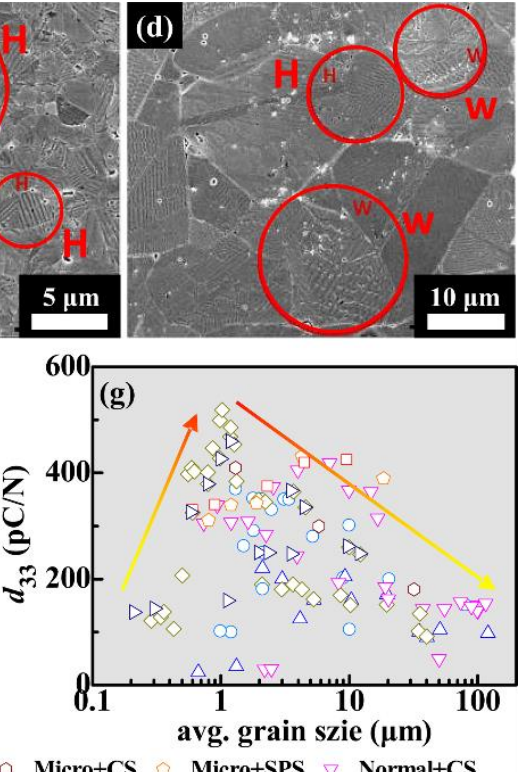

Figure 20. $\mathrm{BaTiO}_{3}$ ceramics prepared by spark plasma sintering with an average grain size (AGS) of (a) $0.8 \mu \mathrm{m}$, (b)

$1.9 \mu \mathrm{m}$, (c) $4.3 \mu \mathrm{m}$, and (d) $18.5 \mu \mathrm{m} .{ }^{162}$ (e) Domain size, (f) permittivity, and (g) $d_{33}$ values of $\mathrm{BaTiO}_{3}$ ceramics with different average grain size. The label of (e-g) indicates how $\mathrm{BaTiO}_{3}$ ceramics were prepared. "Nano" and "Micro" suggest that the raw materials are nano-sized and micro-sized $\mathrm{BaTiO}_{3}$ particles, and "Normal" means that the raw materials are normal $\mathrm{BaCO}_{3}$ and $\mathrm{TiO}_{2}$ powder. "MS", "CS", "TSS" "SPS" and "RC-TSS" represents the microwave sintering, conventional sintering, two-step sintering, spark plasma sintering, and rate-controlled two-step sintering, respectively. "Mixed" manifests the use of two or more different sintering methods. (a-d) Reproduced from ref. 162. with permission from the Springer Nature, Copyright 2015.

Conversely, the modified BT-based ceramics exhibited a different grain size effect compared to pure $\mathrm{BaTiO}_{3}$ ceramics. ${ }^{130,430,431}$ Figure $21(\mathrm{a}, \mathrm{b})$ shows $d_{33}$ and $d_{33}{ }^{*}$ values of $\mathrm{Ba}\left(\mathrm{Ti}_{0.96} \mathrm{Sn}_{0.04}\right) \mathrm{O}_{3}\left(\mathrm{BTS}_{0.04}\right)$ and $\left(\mathrm{Ba}_{0.85} \mathrm{Ca}_{0.15}\right)\left(\mathrm{Zr}_{0.1} \mathrm{Ti}_{0.9}\right) \mathrm{O}_{3}\left(\mathrm{~B}_{0.85} \mathrm{C}_{0.15} \mathrm{~T}_{0.1} \mathrm{Z}_{0.9}\right)$ ceramics varying with the grain size. ${ }^{130,431} \mathrm{BTS}_{0.04}$ ceramics were reported to possess an $\mathrm{O}-\mathrm{T}$ phase coexistence, and $\mathrm{B}_{0.85} \mathrm{C}_{0.15} \mathrm{~T}_{0.1} \mathrm{Z}_{0.9}$ ceramics showed an R-T phase coexistence. ${ }^{54,70,130,431}$ With an increasing AGS, $d_{33}$ of both ceramics increased monotonously; $d_{33} *$ of $\mathrm{BTS}_{0.04}$ ceramics first increased and then reduced; $d_{33} *$ of $\mathrm{B}_{0.85} \mathrm{C}_{0.15} \mathrm{~T}_{0.1} \mathrm{Z}_{0.9}$ 
ceramics increased monotonously. For $\mathrm{BTS}_{0.04}$ ceramics, it was believed that the high $\varepsilon_{\mathrm{r}}$ achieved in fine-grained BTS ceramics was due to the high domain wall density and PNRs; high $d_{33}$ was obtained in coarse-grained ceramics due to a high degree of domain alignment during poling; large electric fieldinduced strain in intermediate-grained ceramics was an outcome of a favorable interplay between constraints from grain boundaries and reversible reorientation of non- $180^{\circ}$ domains and polar nanoregions. ${ }^{431}$ For $\mathrm{B}_{0.85} \mathrm{C}_{0.15} \mathrm{~T}_{0.1} \mathrm{Z}_{0.9}$ ceramics, the monotonously increased $d_{33}$ and $d_{33} *$ were attributed to the enhanced domain switching and reduced residual stress. ${ }^{130}$ Despite the different grain size effects in pure $\mathrm{BaTiO}_{3}$ and modified BT-based ceramics, controlling the grain size is a promising way to enhance the electrical properties of BT-based ceramics because of the synergetic effect of domain structure and residual stress.
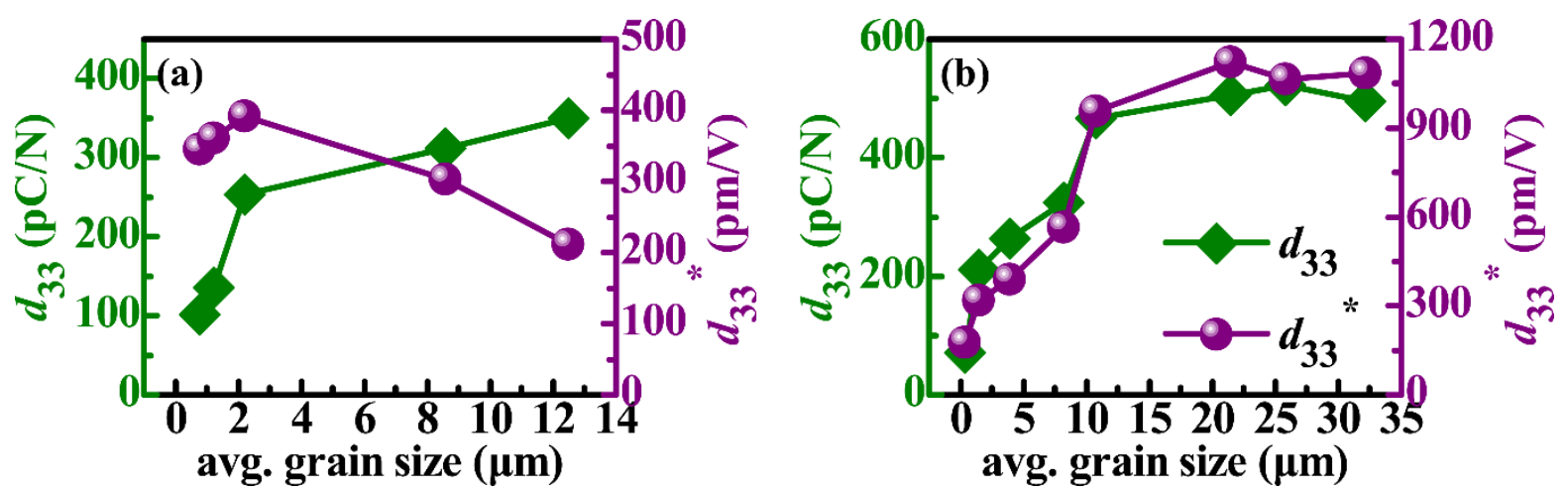

Figure 21. $d_{33}$ and $d_{33}{ }^{*}=S_{\max } / E_{\max }$ of (a) $\mathrm{Ba}\left(\mathrm{Ti}_{0.96} \mathrm{Sn}_{0.04}\right) \mathrm{O}_{3}$ and (b) $\left(\mathrm{Ba}_{0.85} \mathrm{Ca}_{0.15}\right)\left(\mathrm{Zr}_{0.1} \mathrm{Ti}_{0.9}\right) \mathrm{O}_{3}$ ceramics with different average grain sizes. ${ }^{130,431}$ (a) Reproduced from ref. 431. with permission from the Elsevier, Copyright 2019; (b) Reproduced from ref. 130. with permission from the Wiley-VCH, Copyright 2012.

Besides controlling the grain size, the PBE was also widely used to modify the piezoelectric properties of BT-based ceramics. ${ }^{54,65,70}$ The most notable breakthrough is the (1-x)BZT- $x$ BCT ceramic reported by Ren et al. ${ }^{54}$ The corresponding domain structure analysis is displayed in Figure $22(\mathrm{a}-\mathrm{c}) .{ }^{305,} 306$ 0.6BZT-0.4BCT and 0.4BZT-0.6BCT ceramics exhibited the typical $\mathrm{R}$ and $\mathrm{T}$ phase domain structure 
with micron-scale, respectively (see Figure 22(a, c)). 0.5BZT-0.5BCT ceramics showed the micronsized domain lamellas that were comprised of hierarchical nano-domains with a scale of 20-100 nm, as marked by the enlargement of the dash-squared area in Figure 22(b). Besides, Lu et al. also observed similar submicron domains in $0.5 \mathrm{BZT}-0.5 \mathrm{BCT} .{ }^{306}$ Such a unique domain structure was also observed in other lead-free piezoelectric materials and was believed to be the consequence of reduced anisotropy energy in multi-phase coexistence. ${ }^{138,140-143,150,300,445-449}$ Therefore, this domain structure is prone to responding to an external stimulus, such as electric field, benefiting the net piezoelectric properties. In addition, Lu et al. also observed wedge-shaped domains with an interwoven structure of two sets of domains in $0.5 \mathrm{BZT}-0.5 \mathrm{BCT}$ ceramics, which were believed to be the typical $\mathrm{R}$ phase ferroelectric domains. ${ }^{142,150}$ Furthermore, other BT-based ceramics with high piezoelectricity also exhibited the nano-domain structure. For example, $\mathrm{BTS}_{0.11}-0.18 \mathrm{BCT}$ and textured $\mathrm{BCTZ}$ ceramics showed the nanodomains with a scale of 20-40 nm and 20-60 nm, respectively (see Figure $22\left(\mathrm{~d}\right.$, e)). ${ }^{65,73}$ We then collected the relationship between domain size and $d_{33}$ values among several representative BT-based ceramics (see Figure 22(f)). ${ }^{54,65,70}$ The smaller domain size is, the higher the $d_{33}$ value is, which was also observed in KNN-based ceramics (see Figure 9(a)). Such a phenomenon is more distinct in BTbased single crystal. ${ }^{450}$ Wada et al. studied the relationship between domain size and $d_{33}$ values for $<111>$ poled $\mathrm{BaTiO}_{3}$ single crystal. ${ }^{450}$ The results showed that the piezoelectricity was enhanced with the decreasing domain size (see Figure 22(g)). This tendency not only belongs to the lead-free piezoelectric systems but also the lead-based ones. ${ }^{451}$ For example, Lin et al. found a similar tendency in tetragonal PIN-PMN-PT single crystal. ${ }^{451}$ Therefore, an empirical conclusion can be obtained for $\mathrm{KNN}$ - and BT-based ceramics, that is, the smaller the nano-domain is, the higher the $d_{33}$ value is. It also should be mentioned that such a "small" is not infinite. Generally, the stability of the ferroelectric 
phase reduces with decreasing domain size. ${ }^{169}$ The ferroelectric phase may transform to the paraelectric phase below a critical size. ${ }^{169}$ Therefore, controlling the domain size appropriately can achieve higher piezoelectricity.
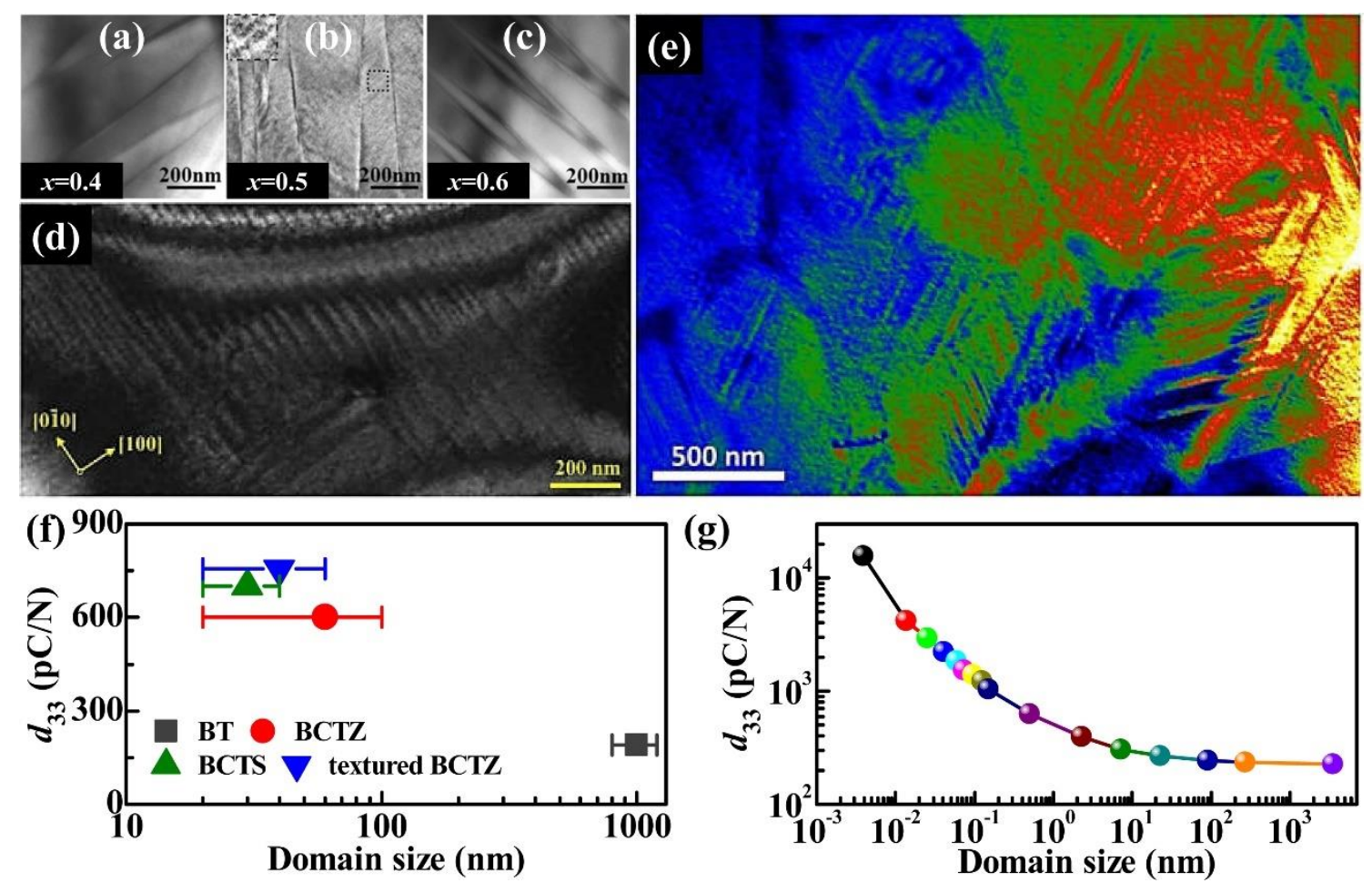

Figure 22. Domain structure of (a-c) BCT- $x$ BZT $\left(x=0.4,0.5\right.$, and 0.6), ${ }^{305}$ (d) textured BCTZ, ${ }^{73}$ and (e) $\mathrm{BTS}_{0.11^{-}}$ 0.18BCT ceramics. ${ }^{65}$ Relationship between domain size and $d_{33}$ in (f) BT-based ceramics and (g) $<111>$ poled BT single crystals. ${ }^{450}$ (a-c) Reproduced from ref. 305. with permission from the American Institute of Physics, Copyright 2011; (d) Reproduced from ref. 73. with permission from the American Chemical Society, Copyright 2017; (e) Reproduced from ref. 65. with permission from the American Chemical Society, Copyright 2018; (g) Reproduced from ref. 450. with permission from the Taylor \& Francis, Copyright 2006.

The above-mentioned domains were observed from unpoled BT-based ceramics. However, in situ electric-dependent variations of domain structure are highly desired for further explaining the physical mechanisms of the enhanced piezoelectric properties in BT-based ceramics. Tan et al. studied the 
variations of nano-domains in 50BZT-50BCT ceramics under an electric field (see Figure 23). ${ }^{357}$ Before applying the electric field, the complicated multi-domain structure, including hierarchical nanodomains and long lamellar domains, were observed (see Figure 23(a)). When the electric field increased up to $1 \mathrm{kV} / \mathrm{cm}$, the domain morphology was significantly changed, suggesting the extensive domain switching activities at this low level of the electric field (see Figure 23(b)). However, the multidomain state was still preserved. As the electric field reached $1.33 \mathrm{kV} / \mathrm{cm}$, no domain walls were observed, and the whole area exhibited a single-domain structure (see Figure 23(c)). However, such a single-domain structure changed into a multi-domain structure again at a higher electric field of 4.5 $\mathrm{kV} / \mathrm{cm}$ (see Figure 23(d)), indicating the instability of the single-domain structure. The corresponding selected area electron diffraction (SAED) patterns were also recorded (see Figure 23(e-g)). Neither detectable changes in the diffraction pattern nor the appearance of superlattice spots were observed. Thus, they attributed this unique single-domain structure to be responsible for the enhanced piezoelectric properties in 50BZT-50BCT ceramics. ${ }^{357}$ 

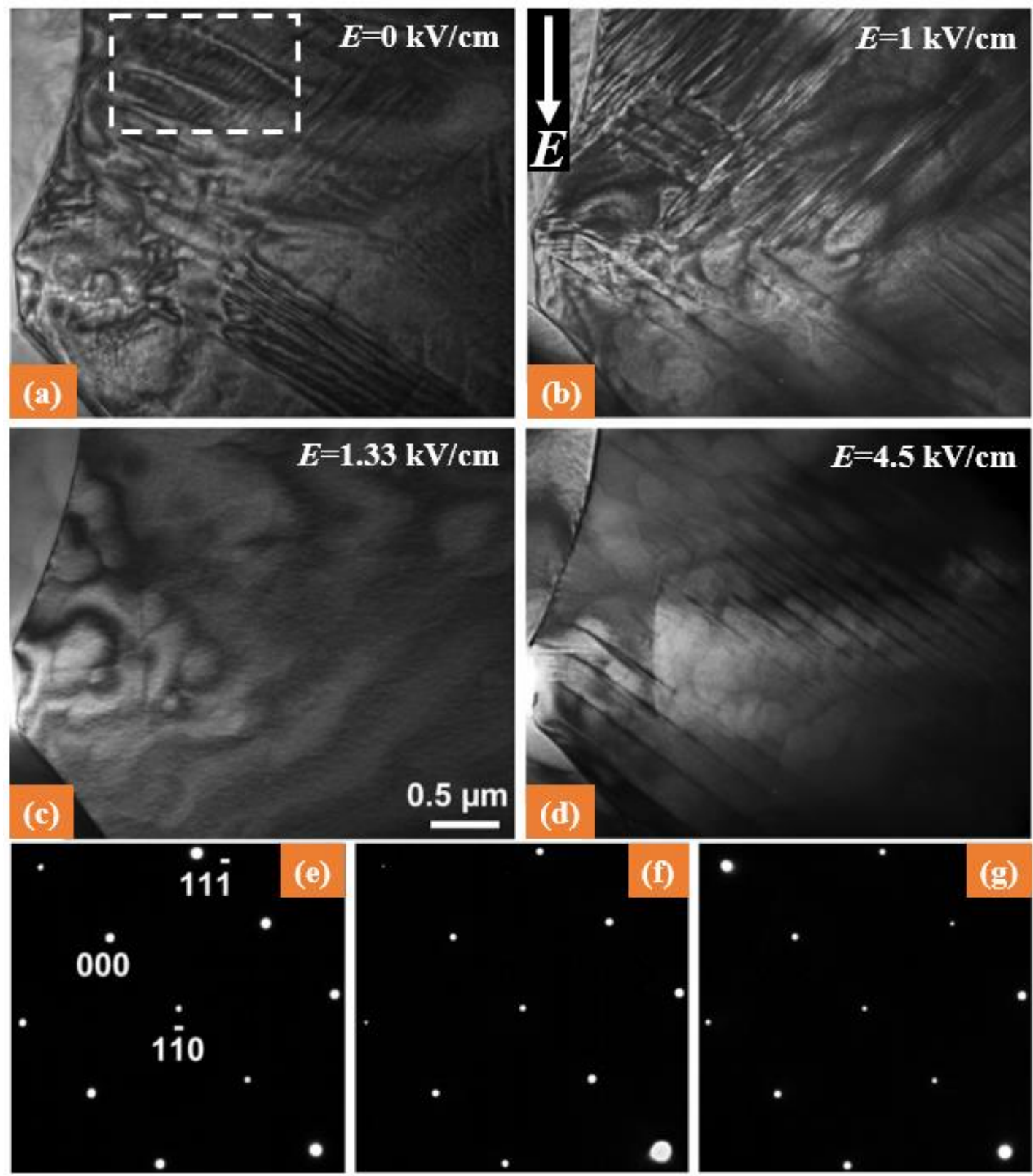

$=1.33 \mathrm{kV} / \mathrm{cm}$
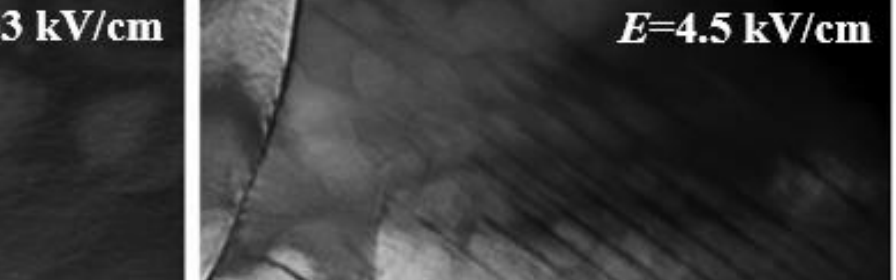

\section{$0.5 \mu \mathrm{m}$}
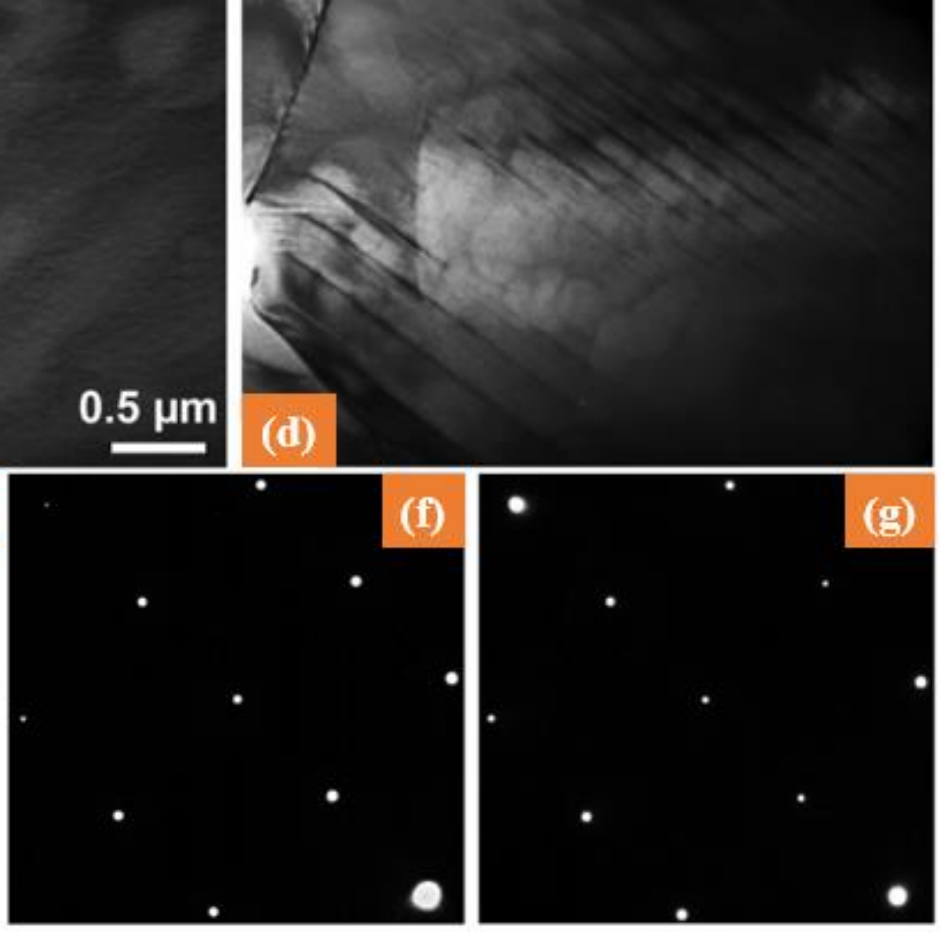

Figure 23. in situ TEM observations of a grain along $<112>$ zone axis in the $0.5 \mathrm{Ba}\left(\mathrm{Zr}_{0.2} \mathrm{Ti}_{0.8}\right) \mathrm{O}_{3}-0.5\left(\mathrm{Ba}_{0.7} \mathrm{Ca}_{0.3}\right) \mathrm{TiO}_{3}$

(50BZT-50BCT) ceramic under electric fields. ${ }^{357}$ Bright-field micrographs at (a) virgin state, (b) $1.00 \mathrm{kV} / \mathrm{cm}$, (c) 1.33

$\mathrm{kV} / \mathrm{cm}$, and (d) $4.50 \mathrm{kV} / \mathrm{cm}$. The direction of the poling field is indicated by the dark arrow in (b). Representative

SAED patterns are recorded at (e) virgin state, (f) $1.33 \mathrm{kV} / \mathrm{cm}$, and (g) $4.50 \mathrm{kV} / \mathrm{cm}$. The white dashed box in Figure

11(a) indicates the location of hierarchical nano-domains. The white arrow in Figure 11(b) shows the direction of the applied electric field. (a-g) Reproduced from ref. 357. with permission from the American Physical Society, Copyright 2014. 
Subsequently, Tan et al. and Zakhozheva et al. carried out the systematic investigations on the domain structure varying with the electric field in (1-x)BZT- $x$ BCT ceramics. ${ }^{358-360}$ The results showed that a multi-domain state was changed into a single-domain state under an increasing electric field through forming an intermediate nano-domain state, regardless of the phase structure of $(1-x) \mathrm{BZT}-x \mathrm{BCT}$ ceramics. Such a transformation was reversible, which suggested that the single-domain state could return to the multi-domain state when removing or reducing the electric field (see Figure 24(a)). ${ }^{358,359}$ The necessary electric field for inducing the single-domain state strongly depended on the composition. ${ }^{358,359}$ The compositions with R phase or near polymorphic phase transition (PPT), such as 70BZT-30BCT and 50BZT-50BCT ceramics, only needed an electric field of $1.33 \sim 2 \mathrm{kV} / \mathrm{cm}$, but the ones with $\mathrm{T}$ phase (e.g., 40BZT-60BCT ceramics) required an electric field as high as $20 \mathrm{kV} / \mathrm{cm}$. However, the single-domain state was metastable, which could be irreversibly changed into a multidomain state again via an intermediate nano-domain state when further increasing the amplitude of the electric field (see Figure 24(b)). ${ }^{358,359}$ The occurrence of the intermediate nano-domain state was due to the local strain gradients and strain incompatibility of adjacent grains. ${ }^{358,359}$ Here, it should be pointed out that although these conclusions were phenomenologically obtained from the observations, the corresponding physical mechanisms also supported the large contribution from the domain switching and domain wall motion. Gao et al. evaluated the extrinsic contribution to the piezoelectric properties of 50BZT-50BCT ceramics by using the Rayleigh analysis at the low electric field. ${ }^{370}$ They found that the extrinsic piezoelectricity, which was associated with the reversible domain wall motion, exhibited the maximum value in the phase transition region, and its contribution for the piezoelectricity enhanced of 50BZT-50BCT ceramics was as high as 67\%, strongly demonstrating the crucial role of 
nano-domains in the piezoelectric properties of BT-based ceramics. ${ }^{370}$

(a)

Reversible transformation:

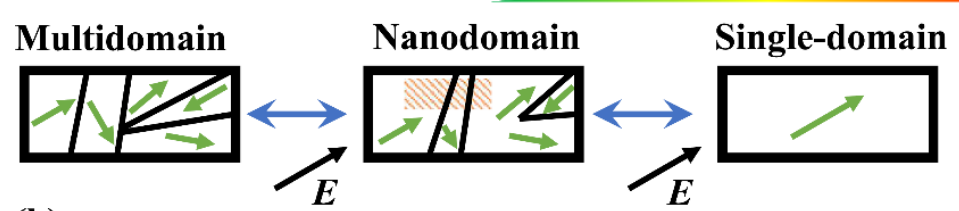

$\boldsymbol{E}$

(b)

$\boldsymbol{E}$

Switching between two multi-domain states:

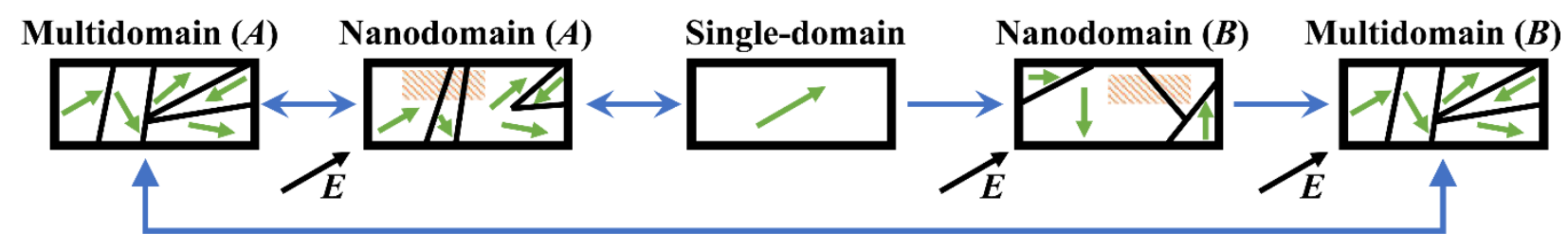

Figure 24. Scheme of domain evolution in BZT- $x$ BCT ceramics under an external electric field. ${ }^{359}$ (a) Reversible domain transformation. (b) Switching between two multi-domain states. (a, b) Reproduced from ref. 359. with permission from the American Physical Society, Copyright 2015.

\subsection{Nano-domain vs. Stability}

\subsubsection{Nano-domain vs. Temperature stability}

Like the reports in KNN-based ceramics, nano-domains also affect the temperature stability of BTbased ceramics. Lu et al. studied the effects of nano-domains on the temperature stability of 50BZT50BCT ceramics by simultaneously considering in situ temperature-dependent domain structure and electrical properties. ${ }^{306}$ Figure $25(\mathrm{a}-\mathrm{h})$ shows the domain structure of 50BZT-50BCT ceramics during heating and cooling. At room temperature (e.g., $25^{\circ} \mathrm{C}$ ), the parallel lamellar domains and wedgeshaped domains with nano-domains inclusions were observed (see Figure 25(a)). When heating from $25{ }^{\circ} \mathrm{C}$ to $60{ }^{\circ} \mathrm{C}$, the wedge-shaped domains significantly reduced, and the nano-domains gradually merged and then transformed into the lamellar domains (see Figure 25(b)). This transformation was induced by the phase transition from the $\mathrm{R}$ phase to the $\mathrm{T}$ phase with increasing temperature. As 
temperature further increased up to $90{ }^{\circ} \mathrm{C}$, ferroelectric domains substantially reduced because of the ferroelectric to paraelectric phase transition (see Figure 25(c)). However, some curved domains and parallel domains were still observed. In particular, some parallel domains even retained at $110{ }^{\circ} \mathrm{C}$ (see Figure $25(\mathrm{~d})$ ). However, no ferroelectric domains were observed at $120^{\circ} \mathrm{C}$ that was much higher than its $T_{\mathrm{c}}$ value $\left(\sim 90{ }^{\circ} \mathrm{C}\right.$ ) (see Figure $\left.25(\mathrm{e})\right)$. When cooling to $90{ }^{\circ} \mathrm{C}$, the lamellar domains with a scale of $100 \mathrm{~nm}$ appeared again (see Figure 25(f)). These lamellar domains gradually emerged into the submicron domains containing nano-domains at $60{ }^{\circ} \mathrm{C}$ (see Figure $25(\mathrm{~g})$ ). Finally, only parallel lamellar domains were observed at $25^{\circ} \mathrm{C}$ after cooling (see Figure 25(h)), indicating the wedge-shaped domains were highly mobile and irreversibly changed varying with temperature.

Then, they related the variations of electrical properties with temperature to the change of domain structure. ${ }^{306}$ When increasing temperature, $d_{33}$ and $k_{\mathrm{p}}$ values gradually reduced before reaching $T_{\mathrm{c}}$ and rapidly reduced to $0 \mathrm{pC} / \mathrm{N}$ after exceeding $T_{\mathrm{c}}$. Meanwhile, ferroelectric hysteresis $(P-E)$ loops of 50BZT-50BCT ceramics were also suppressed with the increasing temperature. Therefore, they attributed the reduced electrical properties to the fading nano-domains. ${ }^{306}$ However, the distinct electrical properties (e.g., $d_{33}$ and remanent polarization $P_{\mathrm{r}}$ ) were still observed when the temperature approached or even exceeded $T_{\mathrm{c}}$, which were ascribed to the retention of ferroelectric domains. ${ }^{306}$ 


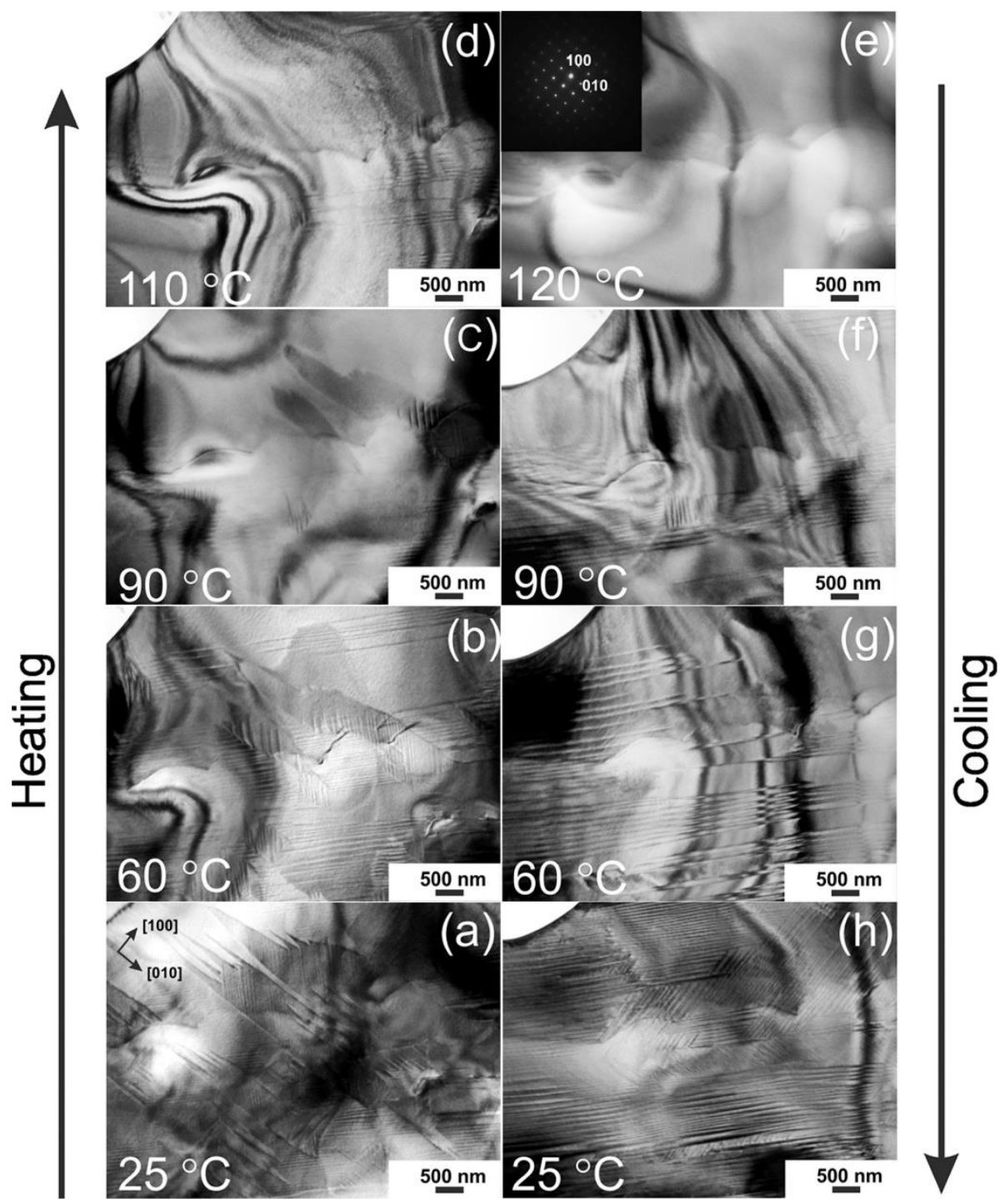

Figure 25. (a-h) in situ temperature-dependent domain structure of 50BZT-50BCT ceramics. ${ }^{306}$ (a) $T=25^{\circ} \mathrm{C}$, (b) $T=60$ ${ }^{\circ} \mathrm{C}$, (c) $T=90^{\circ} \mathrm{C}$, (d) $T=110^{\circ} \mathrm{C}$, (e) $T=120^{\circ} \mathrm{C}$, (f) $T=90^{\circ} \mathrm{C}$, (g) $T=60^{\circ} \mathrm{C}$, and (h) $T=25^{\circ} \mathrm{C}$. (a-h) Reproduced from ref. 306. with permission from the American Institute of Physics, Copyright 2014.

\subsubsection{Nano-domain vs. Fatigue}

Fatigue behavior is also important for practical applications., ${ }^{4,}, 44$ Previous publications mainly focused on the macroscopic electrical properties and the proposed physical models. ${ }^{452,453}$ Recently, Tan et al. revealed the microstructure mechanism of the fatigue process in 50BCT-50BZT ceramics by using an in situ electric-dependent TEM. ${ }^{454,455}$ In the beginning, the application of a voltage of $120 \mathrm{~V}$ 
changed the domain structure from the nano-domains to the submicron-domains. ${ }^{455}$ However, the number of domains significantly reduced at $0 \mathrm{~V}$ after fatiguing $10^{3}$ electric cycles (see Figure 26(a)). The subsequent application of bias of $120 \mathrm{~V}$ made the domain walls disappear, indicating benign mobility (see Figure 26(b)). However, the submicron-domains were replaced by the complex mixture of defect clusters and fragmented domains, as indicated by the white triangles in Figure 26(b). At $0 \mathrm{~V}$ after fatiguing $3 \times 10^{4}$ electric cycles, only B domain survived and the complex mixture became dominating (see Figure 26(c)). At 120 V, B domain retracted (see Figure 26(d)), suggesting the retention of domain wall motion after fatiguing $3 \times 10^{4}$ electric cycles. At $0 \mathrm{~V}$ after fatiguing $5 \times 10^{4}$ electric cycles, B domain disappeared and the grain was filled up with many defect clusters and fragmented domains (see Figure 26(e)). Furthermore, even a bias of $120 \mathrm{~V}$ was applied, no detectable change was observed in the domain morphology throughout the entire observed area (see Figure 26(f)). In other words, the grain's response to the applied voltage was completely suppressed after $5 \times 10^{4}$ unipolar cycles. Meanwhile, some defect clusters were found to have expanded, indicated by the comparison of the areas marked by the white circles in Figure 26(d, f). The variations of domain structure were consistent with the macro electrical properties measured by Zhang et al. ${ }^{456} 2 P_{\mathrm{r}}$ values significantly reduced after fatiguing $10^{4}$ unipolar cycles but remained unchanged during $10^{4}-10^{5}$ unipolar cycles (see Figure 26(g)). Tan et al. then further revealed the interaction of defect clusters and ferroelectric domains by observing the in situ domains after $3 \times 10^{4}$ electric cycles and found that defect clusters were very effective at blocking the growth and expansion of large ferroelectric domains. ${ }^{455}$ Therefore, the deteriorative electrical properties during the fatigue process were attributed to the reduced large ferroelectric domains that were disrupted and replaced by the complex mixture comprising of defect clusters and fragmented domains. The complex mixtures filled up with the whole 
grain and were nonresponsive to applied voltages.
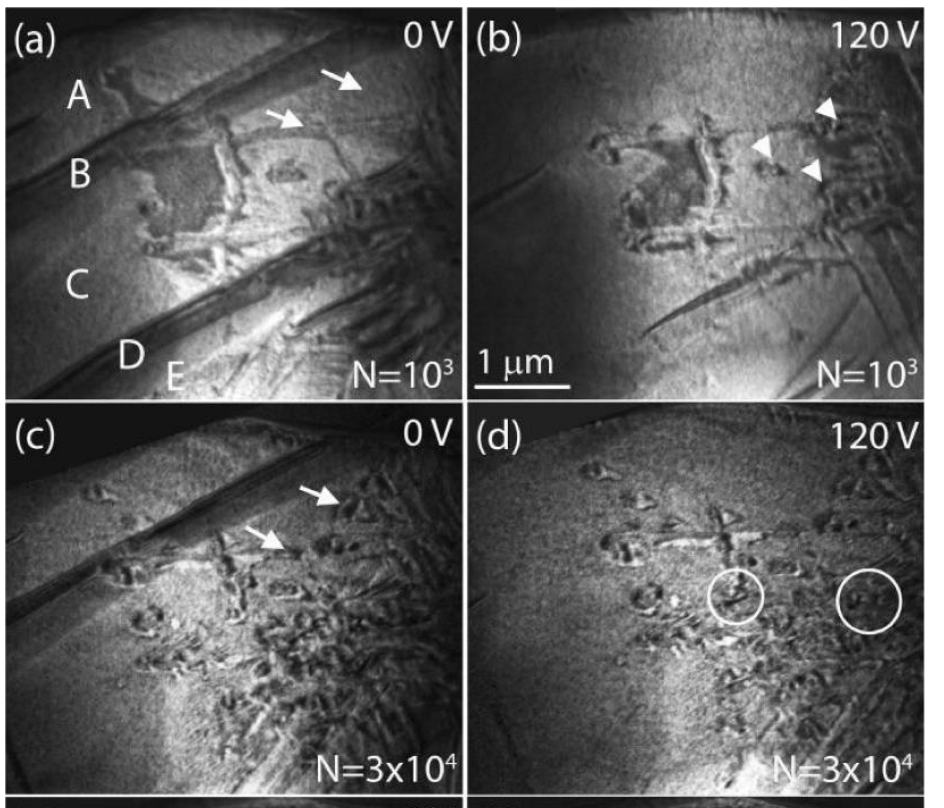

(d)
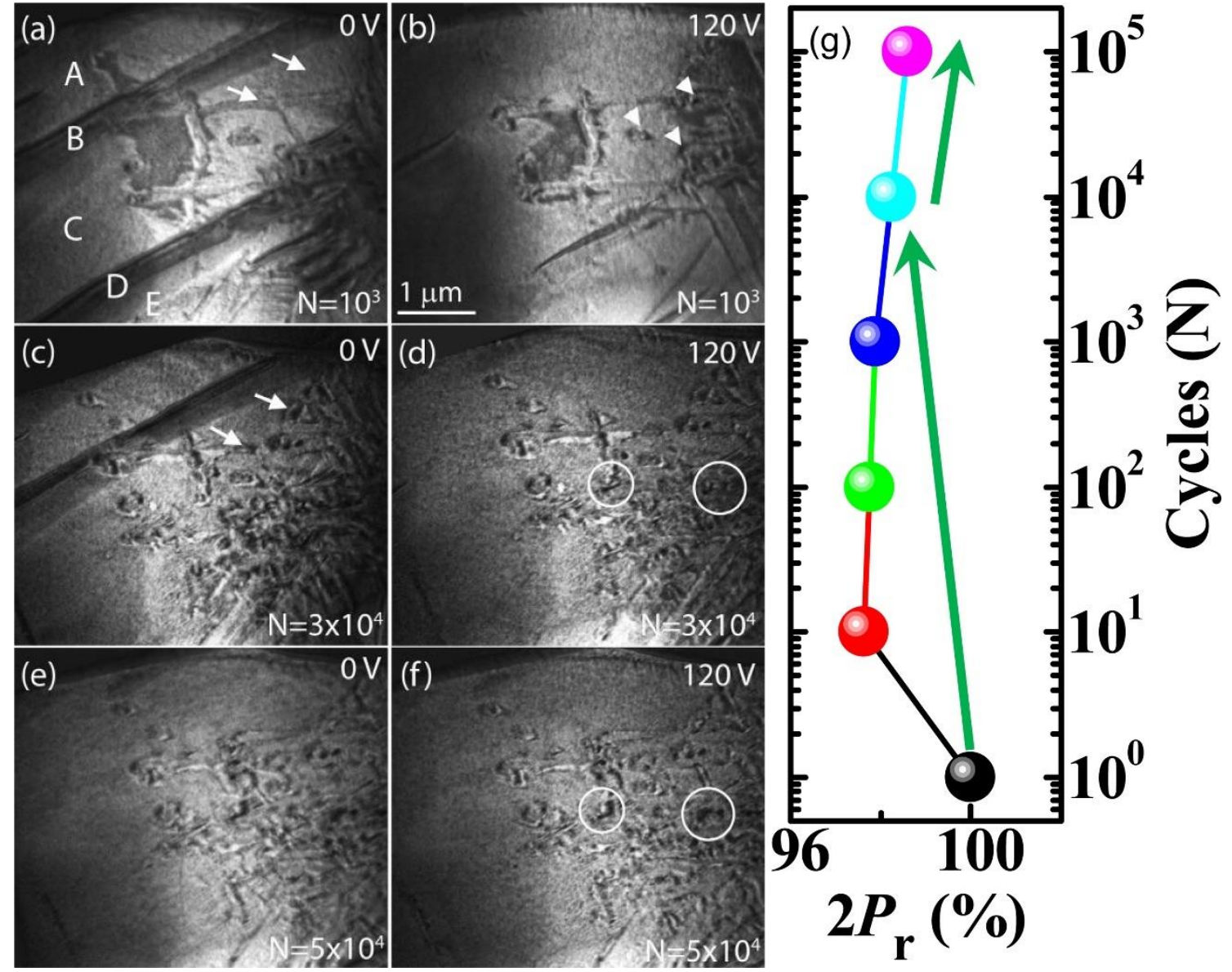

Figure 26. Fatigue analysis of $\mathrm{Ba}_{0.92} \mathrm{Ca}_{0.08} \mathrm{Ti}_{0.945} \mathrm{Zr}_{0.055} \mathrm{O}_{3}$ ceramics. ${ }^{455}$ Bright field images at $0 \mathrm{~V}$ after (a) $10^{3}$, (c) $3 \times 10^{4}$, and (e) $5 \times 10^{4}$ unipolar cycles and at $120 \mathrm{~V}$ after (b) $10^{3}$, (d) $3 \times 10^{4}$, and (f) $5 \times 10^{4}$ unipolar cycles. (g) Normalized $2 P_{\mathrm{r}}$ values of $\mathrm{Ba}_{0.92} \mathrm{Ca}_{0.08} \mathrm{Ti}_{0.945} \mathrm{Zr}_{0.055} \mathrm{O}_{3}$ ceramics as a function of electric cycle. (a-f) Reproduced from ref. 455. with permission from the American Institute of Physics, Copyright 2017.

\subsubsection{Nano-domain vs. Aging}

Aging behavior is also another important factor for evaluating the practical value of a ferro/piezoelectric material. ${ }^{4,12,44}$ By using the same method, Tan et al. studied the microstructure mechanisms of aging in 50BZT-50BCT ceramics. ${ }^{457}$ To reveal the actual situation of the aging process at practical applications, 50BZT-50BCT ceramics were firstly fatigued $10^{6}$ bipolar cycles. The fatigued 
samples exhibited no significant change in $P-E$ loops, as manifested by the almost unchanged $2 P_{\mathrm{r}}$ values (see Figure 27(a, b)). However, the aging process shifted $P$ - $E$ loops downward, reduced $2 P_{\mathrm{r}}$ values exponentially, and induced the increase of the internal bias field $\left(E_{\text {bias }}\right)$ (see Figure 27(b, c)). The occurrence of $E_{\text {bias }}$ originated from the depolarization field that redistributed the oxygen vacancies. The corresponding microstructures are recorded in Figure 27(d-h). After aging for 18 hours, the overall domain structure survived (see Figure 27(d, e)). However, several straight domain walls in the lower part of the observed area were partially disrupted by some complex features, as indicated by the white arrow in Figure 27(e). As aging time increased up to 42 hours, the domain structures were changed substantially. A large number of lamellar domains were disrupted to various degrees and were replaced by complex features (see Figure 27(f)). Furthermore, the area of complex features was increased with increasing aging time, as indicated by the white arrows in Figure 27(e, f). To reveal these complex features, a bias of $108 \mathrm{~V}$ was applied to the same area after aging for 42 hours (see Figure 27(g)). All the large domains disappeared, but the complex features became cleaner. After removing the bias, the large domains reappeared and the complex features were preserved. Therefore, they inferred that these complex features were the mixture of clusters of charged point defects (most likely oxygen vacancies in 50BZT-50BCT ceramics) and small fragmented domains because the oxygen vacancy clusters did not change under a single triangular field cycle. The high local electrostatic and elastic distortion energy at the domain tip accelerated the clustering process of oxygen vacancies. Clustered oxygen vacancies would, in turn, clamp the domain walls and suppress their responses to the applied fields. ${ }^{457}$ Meanwhile, many large domains were disrupted and replaced by the mixtures of oxygen vacancy clusters and fragmented domains after aging (see Figure $27(\mathrm{~d}-\mathrm{f})$ ). Therefore, they ascribed the $E_{\text {bias }}$ and the reduction in $2 P_{\mathrm{r}}$ during the aging process to the microscopic domain wall clamping and domain 
disruption resulted from the redistribution of oxygen vacancies driven by depolarization field. ${ }^{457}$
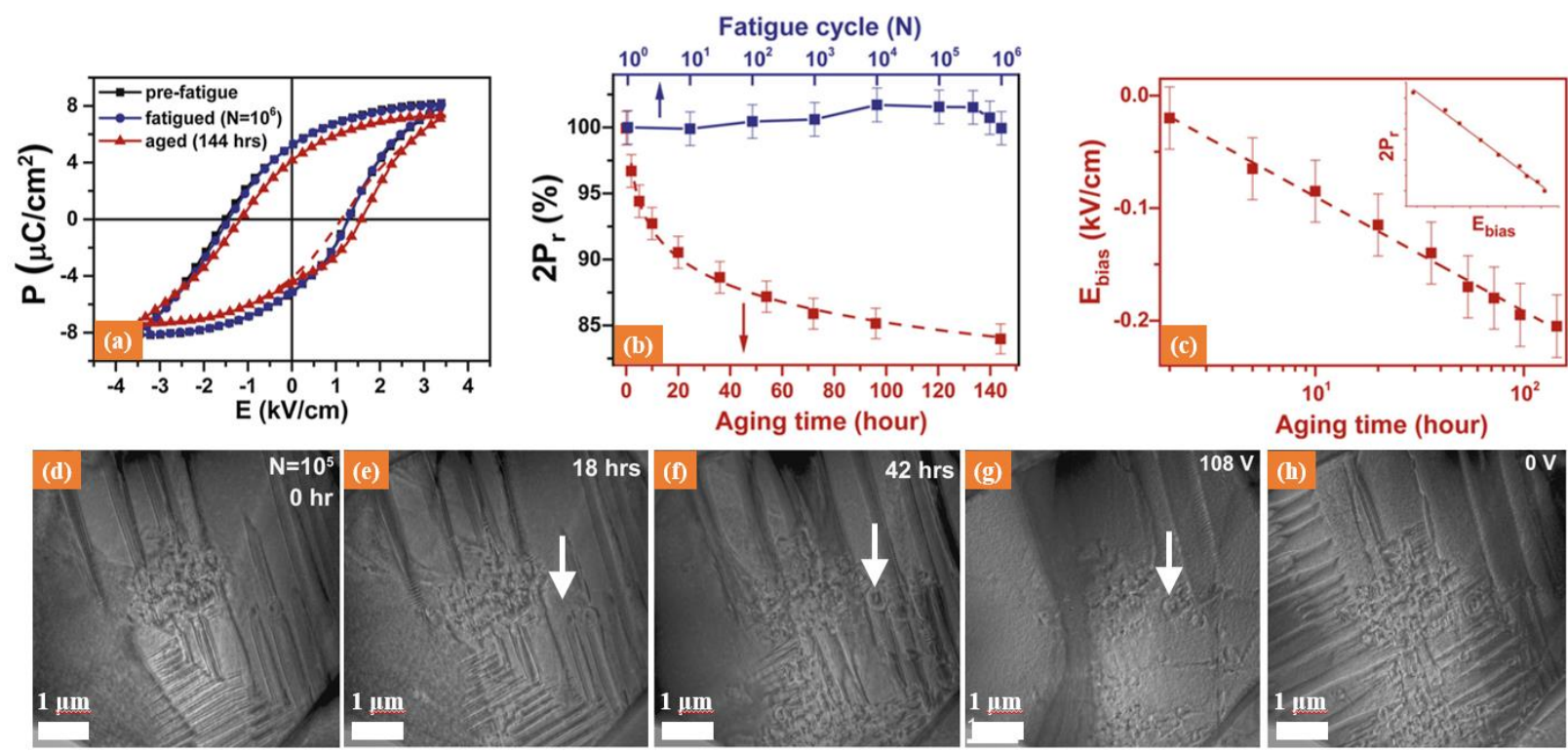

Figure 27. Aging analysis of 50BZT-50BCT ceramics. ${ }^{457}$ (a) $P-E$ loops of 50BZT-50BCT ceramics before fatigue, after fatiguing $10^{6}$ bipolar cycles, and aged for 144 hours. (b) Variations of normalized $2 P_{\mathrm{r}}$ values as a function of the aging time and fatigue cycle. (c) Relationship between aging time and $E_{\text {bias. }}$ The inset of (c) shows the relationship between $E_{\text {bias }}$ and $2 P_{\mathrm{r}}$. Domain structure of 50BZT-50BCT ceramics after $10^{6}$ bipolar cycles aged for (d) 0 hour, (e) 18 hours, (f) 42 hours. Domain structure of (f) at the bias of (g) 108 V and (h) 0 V. (d-e) Reproduced from ref. 457. with permission from the Elsevier, Copyright 2018.

\section{Nano-domains in BNT-based ceramics}

Unlike the situations of KNN- and BT-based ceramics, domain structures in BNT-based ceramics are much more complicated because of the complex phase structure. ${ }^{13}, 15,164,165,216,458-472$ Pure BNT ceramics exhibit moderate piezoelectric properties $\left(d_{33}<200 \mathrm{pC} / \mathrm{N}\right)$ and high $E_{\mathrm{c}}(\sim 70 \mathrm{kV} / \mathrm{cm}) .{ }^{15,458-464}$ Therefore, different methods have been developed to modify the BNT-based ceramics, such as ion substitution, binary or ternary solid solution, ceramic composite, and quenching. ${ }^{13,57,473-478}$ These methods essentially affect the domain structure and subsequently change the macro performance. 
Considering the complexity and vast investigations of BNT-based ceramics' domain structure, here we mainly focused on the variations of nano-domains under the external stimulus (e.g., composition, electric field, and temperature) and their effects on the strain properties and fatigue behavior of BNTbased ceramics. Besides, nano-domains of BNT-based ceramics with other forms (including ceramic composite and core-shell structure) are also reviewed.

\subsection{Nano-domain vs. Phase structure}

\subsubsection{Nano-domains varying with composition}

As we know, binary solid solutions, (1-x)( $\left.\mathrm{Bi}_{0.5} \mathrm{Na}_{0.5}\right) \mathrm{TiO}_{3}-x \mathrm{BaTiO}_{3} \quad(\mathrm{BNT}-x \mathrm{BT})$ and (1$x)\left(\mathrm{Bi}_{0.5} \mathrm{Na}_{0.5}\right) \mathrm{TiO}_{3}-x\left(\mathrm{Bi}_{0.5} \mathrm{~K}_{0.5}\right) \mathrm{TiO}_{3}(\mathrm{BNT}-x \mathrm{BKT})$, are the two of the most studied among these BNT-

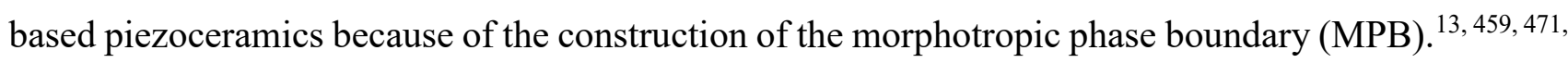
${ }^{479-490}$ Although the phase diagram of BNT- $x$ BT solid solution was depicted by considering XRD patterns and temperature-dependent dielectric properties, the systematic analysis of compositiondependent domain structure in this solid solution is also highly desired. Thus, Tan et al. carried out systematic observations on the domain structure of BNT- $x$ BT ceramics using TEM. ${ }^{491}$ Here we did not show the TEM images because of their excessive numbers, and readers are referred to see them in Ref. 491. The ceramics with $x=0.04$ exhibited the complex domain with a scale of $100 \mathrm{~nm}$, and corresponding SAED patterns indicated an $R 3 c$ phase. At $x=0.06, \sim 40 \%$ of the grain exhibited a coreshell structure and the rest of the grain displayed the nano-domains only. The core still exhibited the complex domain structure, and the shell consisted of nano-domains with faint contrast. SAED patterns proved that the core was an $R 3 c$ phase and the shell possessed a $P 4 b m$ phase. Thus, the addition of $\mathrm{BaTiO}_{3}$ changed the domain structure of BNT ceramics from the complex domain to the nano-domain 
progressively, which explained why only nano-domains were observed in the ceramics with $x=0.07$ and 0.09. These nano-domains exhibited a $P 4 b m$ phase. At $x=0.11$, the sample exhibited the coexistence of a few nano-domains and dominating lamellar domains. The nano-domains had the same $P 4 b m$ phase as the composition with $x=0.06-0.09$, but the lamellar domains showed no superlattice spots, indicating a $P 4 m m$ phase.

In association with the temperature-dependent dielectric properties, they attributed the relaxor behavior in the ceramics with $x=0.07-0.09$ at a temperature below $T_{\mathrm{d}}$ to the existence of nanodomains. ${ }^{491}$ They treated these nano-domains as the characteristic of their newly proposed relaxor antiferroelectric (AFE). AFE nano-domains were embedded in the undistorted cubic matrix. Eventually, a modified composition-dependent phase diagram of BNT- $x$ BT ceramics was drawn in Figure 28. With the increasing content of BT, the domain morphology changed from complex domains to nano-domains, and finally to lamellar domains. A relaxor AFE zone was observed at $x=0.07-0.09$ below $T_{\mathrm{m}}$ (a temperature point where permittivity reaches the maximum value). ${ }^{491}$

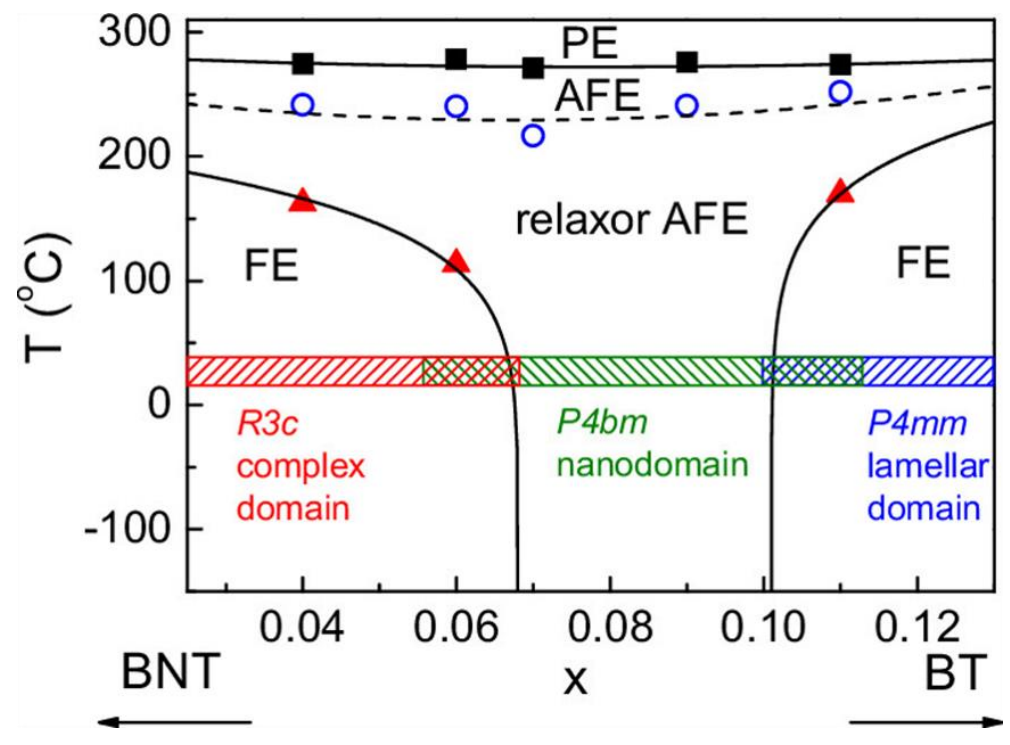

Figure 28. Phase diagram for unpoled BNT- $x$ BT ceramics. Reproduced from ref. 491. with permission from the 
American Institute of Physics, Copyright 2010.

Similar to the situation of BNT- $x$ BT solid solution, $\mathrm{Bi}_{0.5} \mathrm{Na}_{0.5} \mathrm{TiO}_{3}$ and $\mathrm{Bi}_{0.5} \mathrm{~K}_{0.5} \mathrm{TiO}_{3}$ can also form a solid solution at an arbitrary content, which is the process of replacing $\mathrm{Na}^{+}$of $\mathrm{BNT}$ with $\mathrm{K}^{+}$. Pure BNT ceramics exhibit an $R 3 \mathrm{c}$ phase structure at room temperature, and a $P 4 \mathrm{~mm}$ phase structure is reported in pure BKT ceramics. ${ }^{459,}$, 471, 479-482, 492 Thus, an MPB was reported in BNT- $x$ BKT ceramics $(x=0.16$ 0.20), showing an R-T phase coexistence and the enhanced piezoelectric response. ${ }^{459,471,479-482}$ To understand the piezoelectricity enhancement, Otoničar et al. conducted the systematic TEM measurements on the domain structure of BNT- $x$ BKT ceramics $(x=0-1.0) .{ }^{163}$ BNT ceramics exhibited the typical needle-shaped domains with a scale of 5-20 nm in width and 100-200 $\mathrm{nm}$ in length, which formed a square-net pattern. The rhombohedral domain configurations were preserved even at $x=0.15$. As $x$ increased up to 0.20 where an MPB was established, grains were filled up with the well-defined lamellar domains with straight domain walls, similar to that of tetragonal BNT- $x$ BKT $(x=0.25-1.0)$ solid solution. Then, the coexistence of the $\mathrm{R}$ and $\mathrm{T}$ phases was demonstrated by SAED patterns. Finally, only lamellar domains were observed at $x=0.30$ and 1.0 due to the tetragonal phase structure that generally possesses the lamellar $90^{\circ}$ domains. Because of the R-T phase coexistence and lamellar domains, BNT-0.2BKT ceramics locating at MPB exhibited the best performance including piezoelectricity and permittivity (see Figure 29). ${ }^{482}$ 


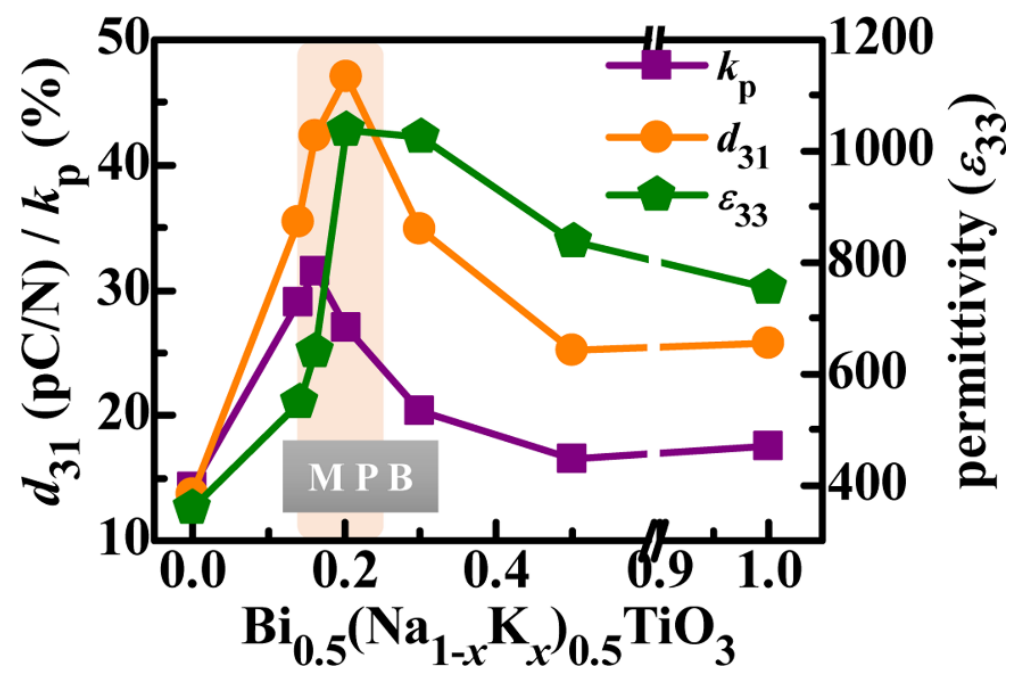

Figure 29. Electrical properties including $d_{31}, k_{\mathrm{p}}$, and permittivity of BNT-xBKT ceramics. ${ }^{482}$

\subsubsection{Nano-domains varying with temperature}

As we know, in situ observations on domain structure provide more direct evidence for explaining the underlying physical mechanisms. Tan et al. measured the domain morphology of BNT-xBT ceramics varying with temperature (see Figure $30(\mathrm{a}-\mathrm{f})$ ). ${ }^{493}$ With increasing temperature, the area of core in the ceramic with $x=0.06$ gradually reduced and completely disappeared at $190{ }^{\circ} \mathrm{C}$ (see Figure $30(\mathrm{a}-\mathrm{c})$ ), resulting in the only nano-domains in the whole grain at $190{ }^{\circ} \mathrm{C}$ (see Figure $30(\mathrm{c})$ ). SAED patterns manifested that the phase structure changed from the coexistence of $R 3 \mathrm{c}$ and $P 4 \mathrm{bm}$ phases to a pure $P 4 b m$ phase. ${ }^{493}$ A similar variation was also observed at $x=0.11$. The increasing temperature progressively reduced the content of lamellar domains, generating a complete nano-domain morphology at $250{ }^{\circ} \mathrm{C}$ (see Figure $30(\mathrm{~d}-\mathrm{f}$ )). The phase structure changed from the coexistence of $P 4 \mathrm{bm}$ and $P 4 \mathrm{~mm}$ phases to a single $P 4 \mathrm{bm}$ phase with increasing temperature. Therefore, they further modified the phase diagram they proposed in Figure 28, as shown in Figure 30(g). For one given composition, the phase transition of BNT-xBT ceramics was gradual. The dielectric anomaly at $T_{\mathrm{d}}$ correlated well with the structural transition to the $P 4 b m$ phase, but the ones at $T_{\mathrm{RE}}$ and $T_{\mathrm{m}}$ did not 
correspond to any structural phase transitions. The tetragonal $(P 4 b m)$-to-cubic $(P m-3 m)$ structural transition occurred progressively within a relatively wide temperature range above $T_{\mathrm{m}}$ where both the size and the population of the $P 4 \mathrm{bm}$ nano-domains reduced with increasing temperature.
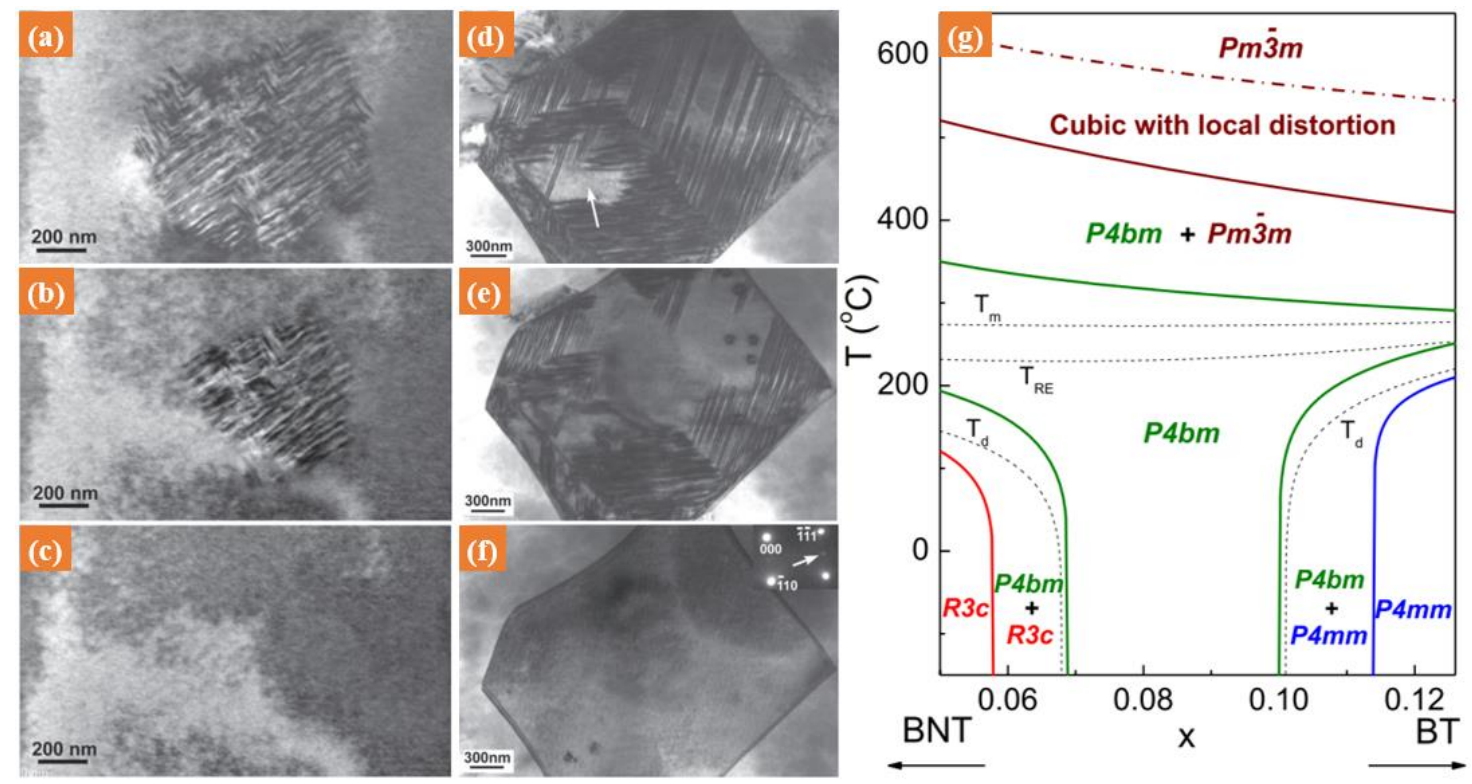

Figure 30. Domain morphology of BNT- $x$ BT ceramics with $x=0.06$ at (a) $25^{\circ} \mathrm{C}$, (b) $140{ }^{\circ} \mathrm{C}$, and (c) $190{ }^{\circ} \mathrm{C}$, and with $x=0.11$ at (d) $25^{\circ} \mathrm{C}$, (e) $200{ }^{\circ} \mathrm{C}$ and (f) $250^{\circ} \mathrm{C}$. (g) Modified phase diagram of BNT- $x$ BT solid solution. Similar to the domain morphology in Figure 16, the sample with $x=0.06$ exhibits the typical core-shell domain structure, and the one with $x=0.11$ shows the coexistence of nano-domains and lamellar domains at room temperature. (a-g) Reproduced from ref. 493. with permission from the Wiley-VCH, Copyright 2011.

Considering the piezoelectricity enhancement at the MPB of BNT- $x$ BKT ceramics, Otoničar et al. also carried out the in situ temperature-dependent TEM measurements on BNT-0.2BKT ceramics. ${ }^{494}$ At room temperature, BNT-0.2BKT ceramics exhibited the lamellar domains with a rhombohedral $(R 3 c)$ $109^{\circ}$ domain structure, as supported by the existence of ${ }^{1 / 2}(o o o)$ superlattice patterns. As discussed above, BNT-0.2BKT ceramics should possess an R-T coexistence phase. The preferred phase structure was believed to originate from the heavy gallium-ion impact for the FIB prepared samples. ${ }^{494}$ The 
preferred tetragonal $(14 \mathrm{~mm})$ phase structure was observed in the conventionally prepared TEM sample because of the mechanical input. When heating up to $180^{\circ} \mathrm{C}$, the lamellar domains and the ${ }^{1 / 2}($ ooo $)$ superlattice patterns disappeared, but the ${ }^{1 / 2}($ ooe $)$ superlattice patterns appeared, indicating the phase transition from $R 3 \mathrm{c}$ phase to $I 4 \mathrm{~mm}$ phase. The domains completely disappeared at $T=300{ }^{\circ} \mathrm{C}$ and only the weak ${ }^{1 / 2}($ ooe $)$ superlattice patterns were observed, indicating the residual $I 4 m m$ phase. When cooling down to the room temperature, the nano-domains that were different from the initial lamellar domains appeared and exhibited both ${ }^{1 / 2}($ ooe $)$ and ${ }^{1 / 2}($ ooo $)$ superlattice patterns, indicating the coexistence of $R 3 \mathrm{c}$ and $I 4 \mathrm{~mm}$ phases.

\subsubsection{Nano-domains varying with electric field}

The domains varying with an external electric field are crucial for explaining the macro variations of electrical properties. Considering the enhanced piezoelectric properties in BNT- $x$ BT ceramics locating at MPB, Tan et al. carried out the in situ observations on domains for the compositions with $x=5.5 \%$, $6 \%$, and 7\% (see Figure 31). ${ }^{495,496}$ For BNT-5.5\%BT ceramics, applying an increasing electric field irreversibly reduced the $P 4 \mathrm{bm}$ nano-domains and increased the $R 3 \mathrm{c}$ large domains, resulting in the $R 3 \mathrm{c}$ domains in the entire area (see Figure 31(a-c)). For one given grain filled by the P4bm nano-domains, BNT-6\%BT ceramics' domains firstly changed into a coexistence of $R 3 \mathrm{c}$ domains and $P 4 \mathrm{~mm}$ lamellar domains and then completely transformed into pure $R 3 c$ domains with an increasing electric field (see Figure $31(\mathrm{~d}-\mathrm{g}))$. Such a transformation was also irreversible. BNT-7\%BT ceramics' domain morphology firstly changed from the entire $P 4 b m$ nano-domains to $P 4 m m$ lamellar domains and subsequently transformed into a coexistence of $R 3 c$ domains and $P 4 \mathrm{~mm}$ lamellar domains (see Figure 31(h-k)). in situ electric-dependent XRD pattern of BNT-7\%BT ceramics also supported the phase transition from a pseudo-cubic to tetragonal symmetry. ${ }^{497}$ Besides, the corresponding phase structure 
of these three compositions varying with an increasing electric field was summarized in Figure 31(1).

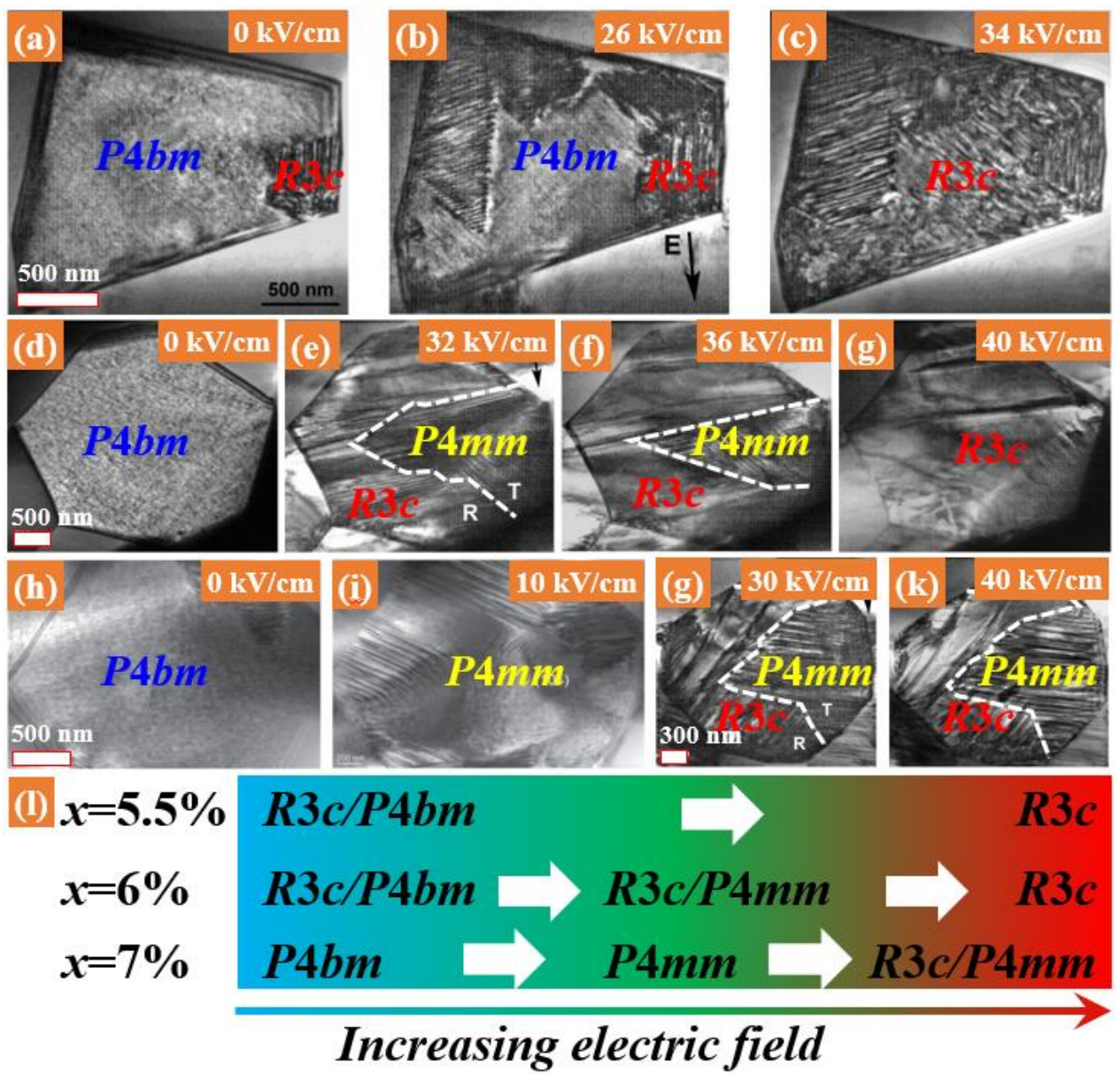

Figure 31. Domain morphology of BNT- $x$ BT ceramics under an increasing electric field. ${ }^{496}(\mathrm{a}-\mathrm{c}) x=5.5 \%,(\mathrm{~d}-\mathrm{g}) x=6 \%$, and (h-k) $x=7 \%$. (l) Phase structure of these three compositions under an increasing electric field. (a-k) Reproduced from ref. 496. with permission from the American Physical Society, Copyright 2012.

Subsequently, Tan et al. proposed a modified phase diagram for the BNT-xBT material system by simultaneously considering composition change, piezoelectric properties (e.g., $d_{33}$ values) and electric field (see Figure 32). ${ }^{496}$ The external electric field higher than $30 \mathrm{kV} / \mathrm{cm}$ created a new MPB at $x=6$ $7 \%$ (see Figure 32(e)). For BNT-5.5\%BT ceramics, $d_{33}$ reached the maximum value of $120 \mathrm{pC} / \mathrm{N}$ when the domain structure completely transformed into the $R 3 c$ domains (see Figure $32(\mathrm{a})$ ). For BNT-6\%BT ceramics, $d_{33}$ only reached the maximum of $131 \mathrm{pC} / \mathrm{N}$ within the created MPB in which both $R 3 c$ 
domains and P4mm lamellar domains coexist (see Figure 32(b)). BNT-7\%BT ceramics' $d_{33}$ monotonously increased with the electric field because of the retention of created MPB consisting of $R 3 \mathrm{c}$ domains and $P 4 \mathrm{~mm}$ lamellar domains at the high electric field, resulting in a maximum value of $167 \mathrm{pC} / \mathrm{N}$ at $65 \mathrm{kV} / \mathrm{cm}$ (see Figure 32(c)). As the electric field was $65 \mathrm{kV} / \mathrm{cm}$, BNT-7\%BT ceramics exhibited the highest $d_{33}$ value, but BNT-5.5\%BT ceramics possessed the lowest one (see Figure 32(d)). Therefore, the enhanced piezoelectric properties in BNT- $x \mathrm{BT}(x=5.5-7 \%)$ ceramics were ascribed to the creation of MPB because of the electric-induced irreversibly ferroelectric-to-ferroelectric phase transition.

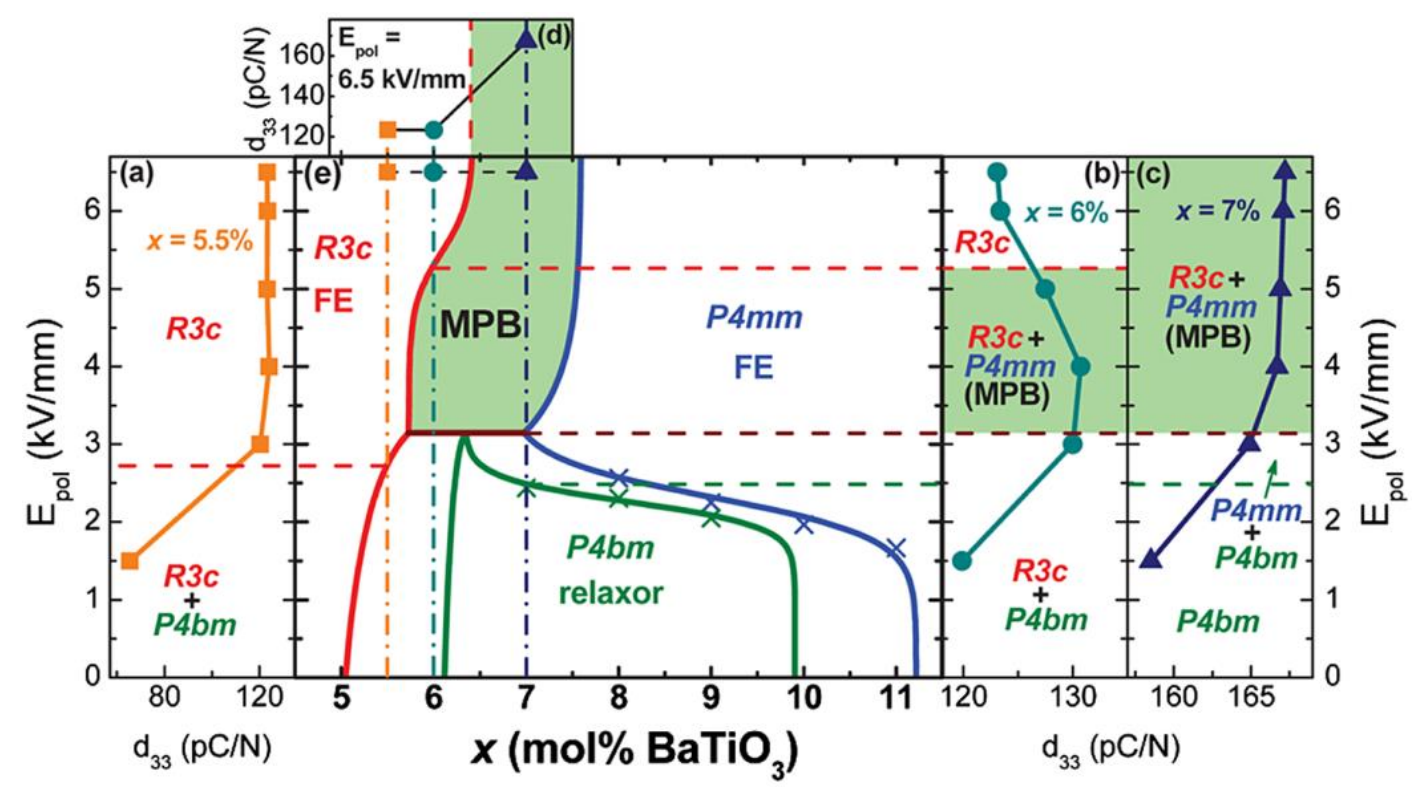

Figure 32. $d_{33}$ as a function of electric field is displayed for BNT- $x$ BT ceramics with (a) $x=5.5 \%$, (b) $x=6 \%$, and (c) $x=7 \%$. (d) $d_{33}$ as a function of composition at $65 \mathrm{kV} / \mathrm{cm}$. (e) The proposed electric field ( $\left.E_{\mathrm{pol}}\right) v s x$ phase diagram for BNT- $x \mathrm{BT}$. FE represents ferroelectric. The electric-field-induced $R 3 c / P 4 m m$ MPB giving rise to enhanced piezoelectricity is emphasized with green shading. (a-e) Reproduced from ref. 496. with permission from the American Physical Society, Copyright 2012.

Considering the two preferred phase structures of BNT-0.2BKT samples that are respectively prepared 
by the conventional method and the FIB method, Otoničar et al. conducted the in situ electricdependent TEM measurements on both samples. ${ }^{494}$ Figure 33(a) shows the domain structure of the conventionally prepared BNT-0.2BKT sample. The typical lamellar domains with a $\mathrm{T}(\mathrm{I} 4 \mathrm{~mm})$ phase structure were observed, which was proved by the existence of ${ }^{1 / 2}($ oeo $){ }^{/ 1 / 2}(e o o)$ superlattice patterns. These lamellar domains exhibited a characteristic $90^{\circ} a$-a type of tetragonal splitting of the main reflections and the super-lattice reflections, as manifested by the SAED 1. Besides, some undefined domains without the clear/straight domain walls were also observed (as marked by the white arrows), which came from the $90^{\circ} a-c$ type of tetragonal twinning based on the additional splitting of the main reflections. When increasing the electric field, domains were re-orientated along the direction of the electric field at the expense of $90^{\circ} a-a$ domains because their polar axes were perpendicular to the electric field. Also, the curved/weakly defined domain walls from the $90^{\circ} a$-c twinning re-orientated along the electric field. Thus, the new domains gradually occupied a large portion of the whole grain. At $E=4.5 \mathrm{kV} / \mathrm{mm}$, some narrowly striped domains occurred (marked by the white arrow in Figure 33(b)). Finally, the whole grain consisted of several single-domain regions that were separated by the curved domain walls (see Figure 33(c)). In particular, the triple-split of the main reflections was still observed at $E=8 \mathrm{kV} / \mathrm{mm}$, indicating three kinds of $90^{\circ}$ tetragonal domain variants were preserved. Meanwhile, only ${ }^{1 / 2}($ ooe $)$ superlattice reflections, which corresponded to the third $90^{\circ}$ domain variant, were observed at $E=8 \mathrm{kV} / \mathrm{mm}$, indicating the electric-induced phase transition from $I 4 \mathrm{~mm}$ to $P 4 \mathrm{bm}$. 

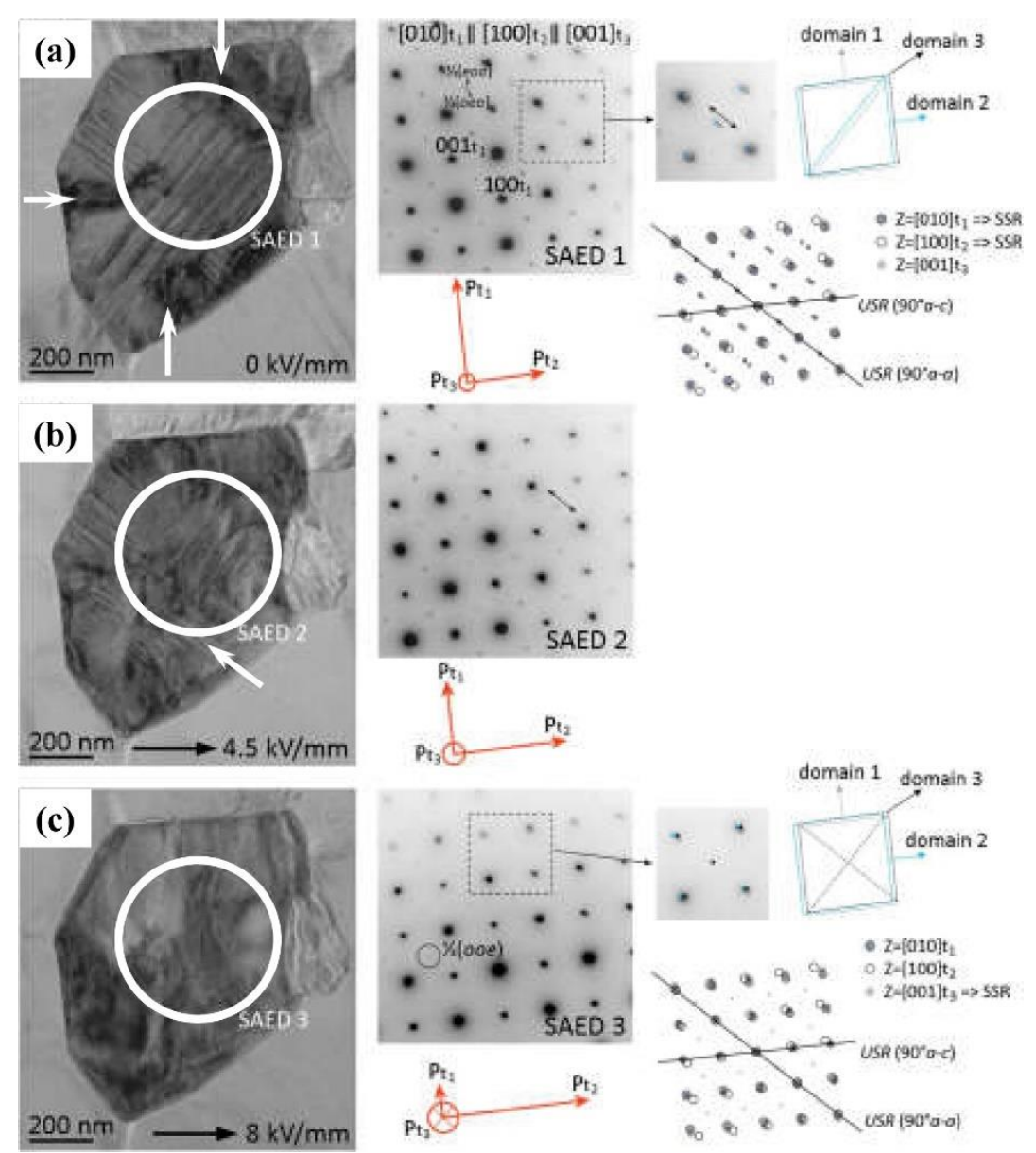

Figure 33. in situ electric-dependent TEM observation of BNT-0.2BKT sample prepared by the conventional method.

(a) $E=0 \mathrm{kV} / \mathrm{mm}$, (b) $E=4.5 \mathrm{kV} / \mathrm{mm}$, and (c) $E=8 \mathrm{kV} / \mathrm{mm}$. The black arrow indicates the direction of the applied electric field. The inset is the corresponding SAED pattern of the area within the white circle and "USR" represents the unsplit row of reflections. " $a-a$ " and " $a-c$ " notations define the relative orientation of the twinned domains toward the viewing direction. " $a$ - $a$ " means that the domains are observed along their $a$-axes and perpendicular to their $c$ axes, while " $a-c$ " indicates that one of the twinned domains is observed along its $a$-axis and another one is observed along the $c$-axis. (a-c) Reproduced from ref. 494. with permission from the Elsevier, Copyright 2017.

Figure 34(a) shows the domain structure of the BNT-0.2BKT sample that was prepared by the FIB method. ${ }^{494}$ The typically wide lamellar domains with an $R 3 c$ phase were observed. With an increasing electric field, the narrowly striped domains emerged (see area 2 in Figure 34(b)). Some undefined 
domains were also observed (see area 3 in Figure 34(b)), which was possibly undergoing the process of re-orientation. As the electric field further increased up to $4 \mathrm{kV} / \mathrm{mm}$, the undefined domains also changed into the narrowly striped domains like those of area 2. In particular, the black arrows in Figure 34(c) indicated some wider lamellar domains, implying the initial formation of a single-domain zone. Besides, all SAED patterns showed the characteristic rhombohedral splitting despite the weak superlattice reflections.

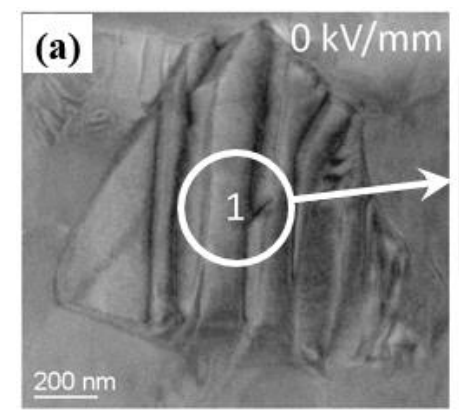

$1[\overline{1} \overline{2} 1] t_{1} \|[\overline{1} \overline{1} 2] t_{2}$
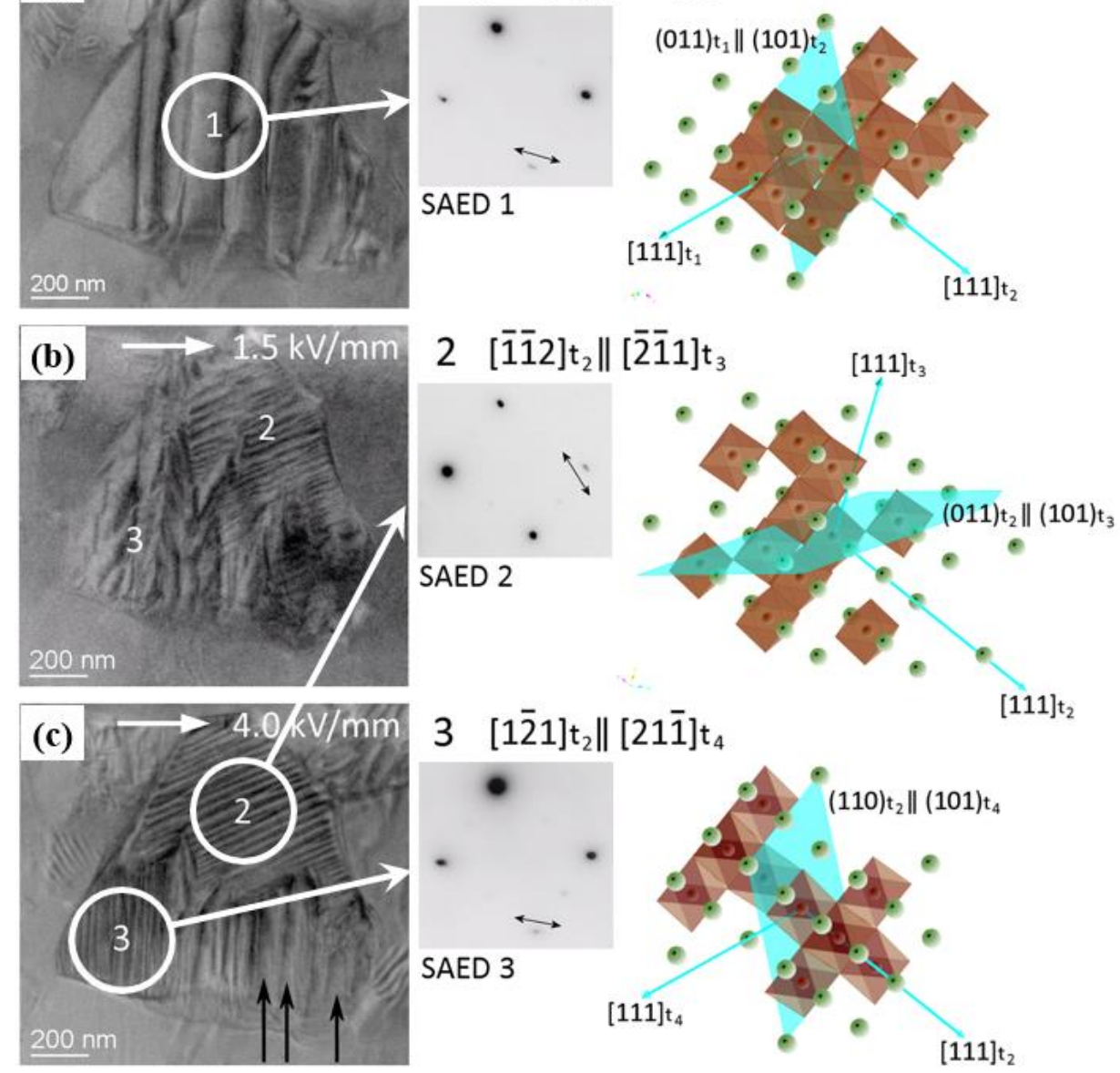

Figure 34. in situ electric-dependent TEM observation of BNT-0.2BKT sample prepared by the FIB method. (a) $E=0$ $\mathrm{kV} / \mathrm{mm}$, (b) $E=1.5 \mathrm{kV} / \mathrm{mm}$, and (c) $E=4 \mathrm{kV} / \mathrm{mm}$. The direction of the electric field is indicated by the white arrow on the top. (a-c) Reproduced from ref. 494. with permission from the Elsevier, Copyright 2017.

Based on the observations above, Otoničar et al. proposed a scheme to describe the domain variations 
of BNT-0.2BKT ceramics with the applied stress and electric field (see Figure 35). ${ }^{494}$ The initial BNT0.2BKT ceramic was full of nano-domains (see Figure 35(a)), which were changed into the lamellar domains by the applied stress (see Figure 35(b)). With an increasing electric field, the domains started to re-orientate along the direction of the applied electric field at $E<E_{\mathrm{c}}$ (see Figure 35(c)). Some narrowly striped domains occurred as an intermediate reorientation step during the electrical poling. As the electric field exceeded $E_{\mathrm{c}}$, the newly developed domains started to align with the direction of the applied electric field and merged into a single-domain zone (see Figure 35(d)). Finally, some relicts of other domains were also observed because of the residual stress (see Figure 35(d)). Therefore, in situ electric-dependent TEM measurements on BNT-0.2BKT ceramics demonstrated the electricinduced phase transition and domain re-orientation as the main origin of the piezoelectricity enhancement at MPB.

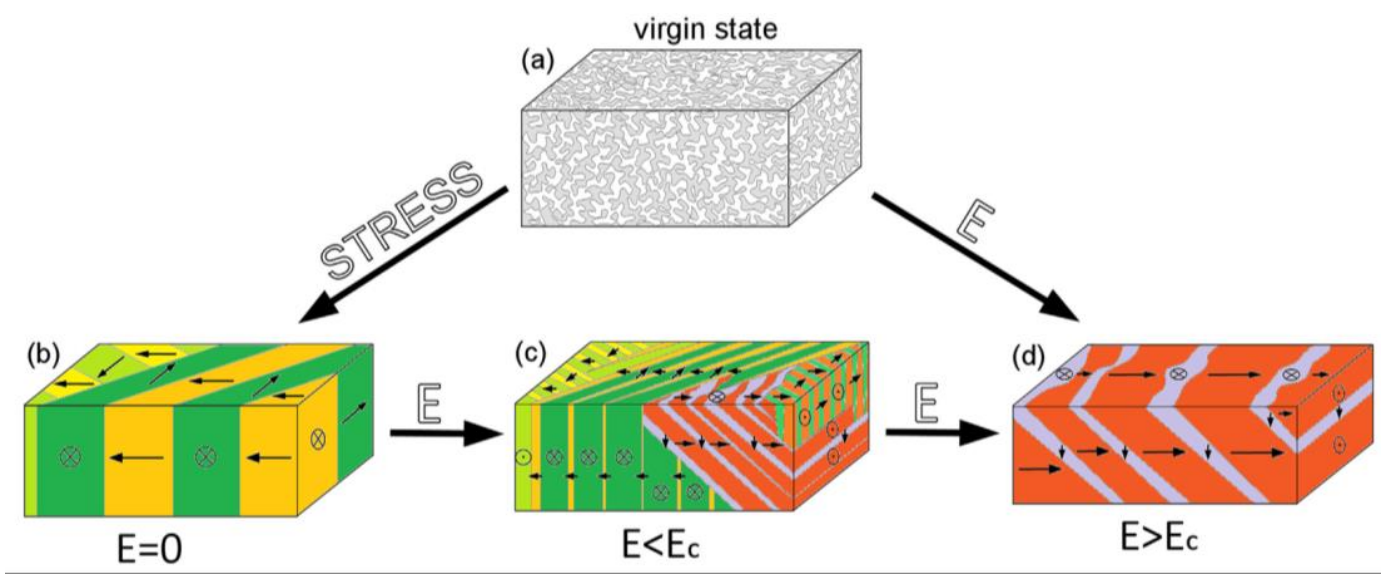

Figure 35. Schematic model illustrating the domain-formation/reorientation process with applied stress and electric field in the NBT-0.2KBT ceramics. (a) Virgin state, (b) state after applying stress, (c) state at $E<E_{\mathrm{c}}$, and (d) state at $E>E_{\text {c. }}$ (a-d) Reproduced from ref. 494. with permission from the Elsevier, Copyright 2017.

\subsection{Nano-domain vs. Strain properties}

BNT-based ceramics are well-known for their excellent electric-induced strain properties. ${ }^{13-15,458}$ 
Nano-domains were reported to greatly affect the strain properties because of the domain switching and domain wall motion under external stimulus (e.g., electric field and stress). The widely studied material systems for high strain properties are $\left(\mathrm{Bi}_{0.5} \mathrm{Na}_{0.5}\right) \mathrm{TiO}_{3}-\mathrm{BaTiO}_{3}-\left(\mathrm{K}_{0.5} \mathrm{Na}_{0.5}\right) \mathrm{NbO}_{3}(\mathrm{BNT}-\mathrm{BT}-$ $\mathrm{KNN})$ and $\left\{\left(\mathrm{Bi}_{0.5}\left(\mathrm{Na}_{0.84} \mathrm{~K}_{0.16}\right)_{0.5}\right)_{0.96} \mathrm{Sr}_{0.04}\right\}\left(\mathrm{Ti}_{1-x} \mathrm{Nb}_{x}\right) \mathrm{O}_{3}(\mathrm{BNT}-x \mathrm{Nb})$ ceramics. ${ }^{58,498-502}$ In this part, the effects of nano-domains on strain of BNT-based ceramics focused on these two material systems.

The high strain properties of the BNT-BT-KNN system were firstly reported by Zhang et al. ${ }^{499-502}$ At that time, they attributed the enhanced strain properties to the electric-induced antiferroelectric-toferroelectric phase transition and domain reorientation. Subsequently, Dittmer et al. systematically analyzed the related physical mechanisms of BNT-BT- $x$ KNN ceramics at the viewpoint of the nanoscale by using PFM. ${ }^{503}$ Figure 36(a, b) shows the bipolar strain curves and related coefficients of $(1-x)(0.94 \mathrm{BNT}-0.06 \mathrm{BT})-x \mathrm{KNN}$ ceramics. The maximum usable strain firstly increased and then reduced with an increasing $x$, reaching the maximum value at $x=0.03$ (see Figure $36(\mathrm{~b})$ ). To reveal the behind physical mechanisms, the compositions with $x=0-0.03$ were written by a bias voltage of $-20 \mathrm{~V}$ to fully re-orientate the nano-domains (see Figure $36(\mathrm{~d} 1$, e $1, \mathrm{f1})$ ). Then, a bias voltage of $+20 \mathrm{~V}$ was applied at the center of the selected area (see Figure 36(d1, e1, f1)). The distinct contrast was observed when immediately removing the bias voltage (see Figure 36(d1, e1, f1)). After removing the bias voltage for 66 minutes, the ceramics with $x=0$ still exhibited the apparent contrast, but the ones with $x=0.03$ displayed no contrast (see Figure $36(\mathrm{~d} 2, \mathrm{e} 2, \mathrm{f} 2)$ ). The piezo-response of Figure $36(\mathrm{~d} 1-\mathrm{f} 2)$ was collected and normalized in Figure 36(c). The nano-domains of ceramics with $x=0.03$ rapidly switched back to the initial state after removing the bias voltage, indicating the fast relaxor behavior. Therefore, PFM experiments further demonstrated that the enhanced strain properties in BNT-BT-KNN ceramics 
were attributed to the reversible antiferroelectric-ferroelectric transition.
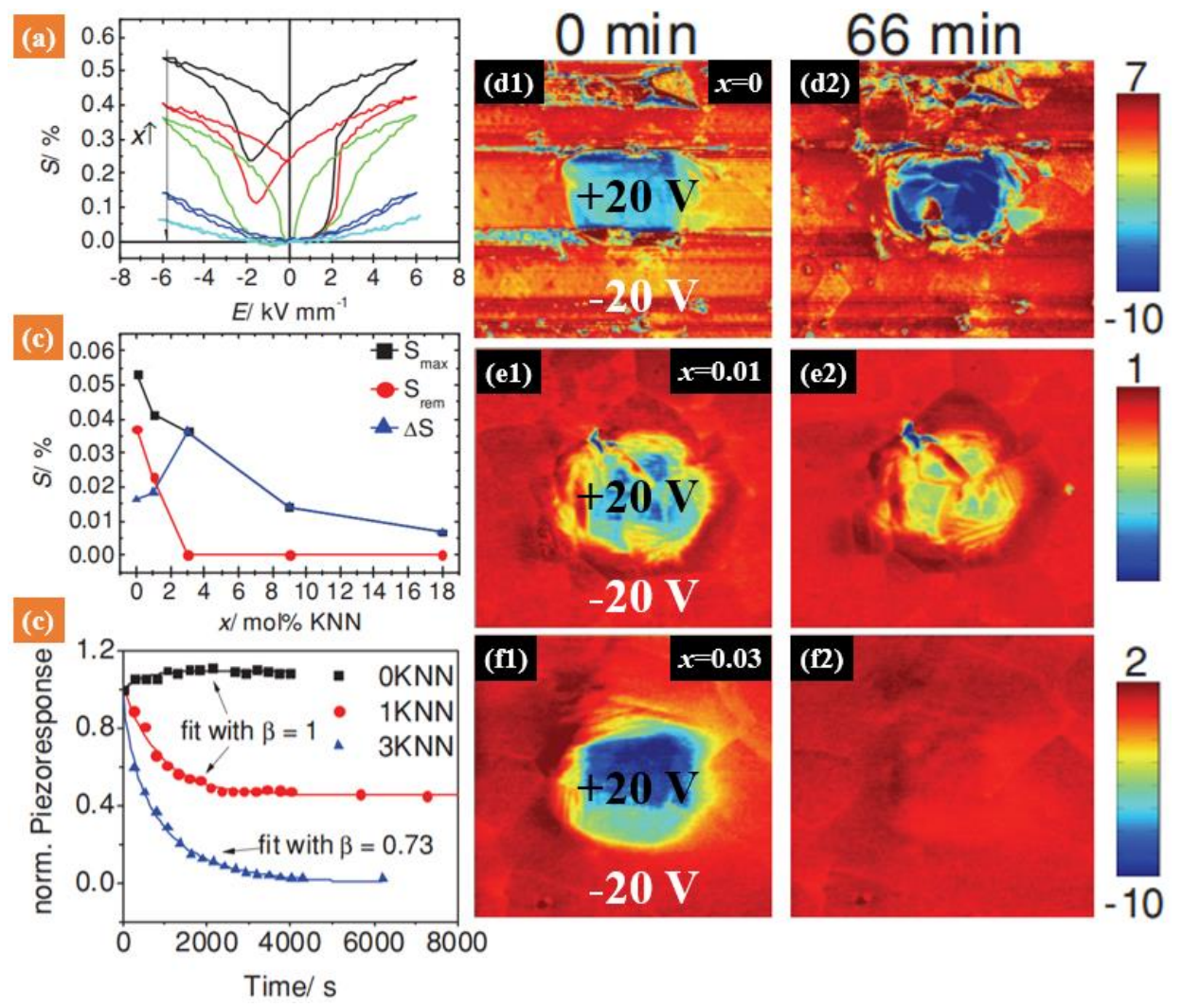

Figure 36. (a) Bipolar strain curves and (b) strain properties (e.g., maximum strain $S_{\max }$, remanent strain $S_{\text {rem, }}$ and usable strain $\left.\Delta S=S_{\max }-S_{\text {rem }}\right)$ of $(1-x)(0.94 \mathrm{BNT}-0.06 \mathrm{BT})-x \mathrm{KNN}$ ceramics. (c) The normalized piezoresponse in (d1-f2). Out-of-plane PFM (OP-PFM) images of $(1-x)(0.94 \mathrm{BNT}-0.06 \mathrm{BT})-x \mathrm{KNN}$ ceramics with (d1-d2) $x=0$, (e1-e2) $x=0.01$, and (f1-f2) $x=0.03$ after writing domain for $0 \mathrm{~min}$ and $66 \mathrm{~min}$. (a-f2) Reproduced from ref. 503. with permission from the Wiley-VCH, Copyright 2012.

In addition, Tan et al. used in situ electric-dependent TEM to further confirm the reversible phase transition and domain switching in BNT-BT-KNN ceramics (see Figure 37). ${ }^{504}$ At the origin state, no obvious domain structure was observed (see Figure 37(a)). Then, an electric field of $25 \mathrm{kV} / \mathrm{cm}$ induced the occurrence of lamellar domains (see Figure 37(b, c)). After removing the electric field, the lamellar domains disappeared (see Figure 37(d)). Therefore, in situ TEM experiments further proved the 
reversible antiferroelectric-ferroelectric phase transition in BNT-BT-KNN ceramics on the application of an electric field.
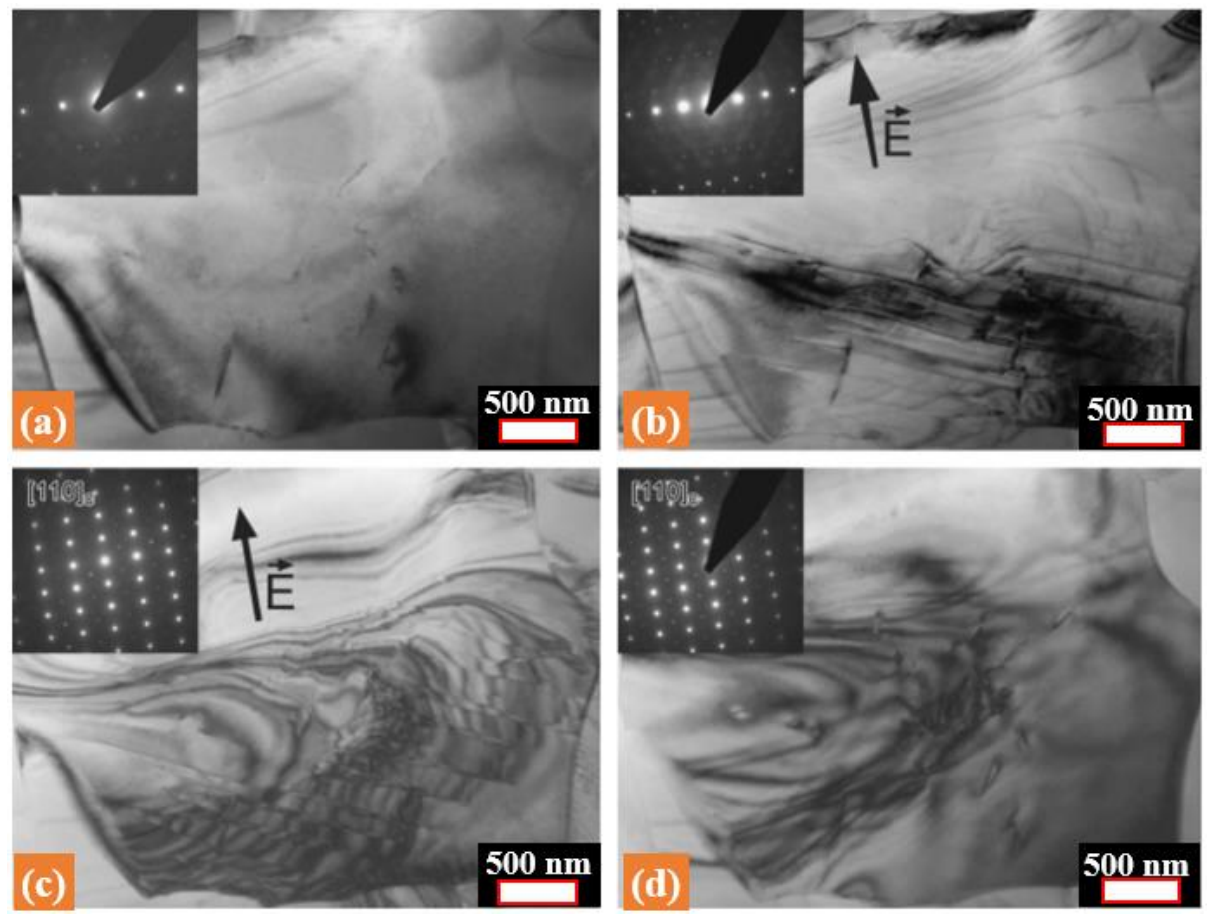

Figure 37. Domain morphology of 0.91BNT-0.06BT-0.03KNN ceramics (a) before applying the electric field, (b-c) at $E=25 \mathrm{kV} / \mathrm{cm}$, and (d) after removing the electric field. The black arrow indicates the direction of the electric field. (a-d) Reproduced from ref. 504. with permission from the Wiley-VCH, Copyright 2010.

BNT-2.5Nb ceramics were reported to possess a giant strain of $0.65-0.70 \%$ at a relatively low electric field of $50 \mathrm{kV} / \mathrm{cm}$ (see Figure $38(\mathrm{a})$ ). ${ }^{58}$ To reveal the physical mechanisms of enhanced strain properties, Tan et al. carried out the in situ electric-dependent TEM measurements on BNT-2.5Nb ceramics (see Figure $38(\mathrm{~b}-\mathrm{g})$ ). At the origin state (e.g., $\mathrm{Z}_{1}$ ), the sample exhibited the phase coexistence of $R 3 c$ and P4bm phases (see Figure 38(h)). The whole grain was occupied by the nano-domains (see Figure 38(b)). As the electric field increased up to $Z_{1}$, the internal of grain started to generate the lamellar domains (see Figure 38(c)). The corresponding strain reached a relatively high level (see Figure 38(a)). At the point of $Z_{2}$, the grain was filled up with the submicron lamellar domains and the corresponding strain 
reached the maximum value (see Figure $38(\mathrm{a}, \mathrm{d})$ ). The SAED pattern exhibited only an $R 3 c$ phase (see Figure 38(i)). Upon the decrease of the electric field, the lamellar domains and the corresponding strain gradually reduced (see Figure 38(a, e, f). Finally, a small number of lamellar domains were retained after removing the electric field (see Figure 38(g)), indicating the partly reversible phase transition. Therefore, they ascribed the giant strain to the phase transitions between the ergodic relaxor phases in the form of mixed $R 3 c$ and $P 4 b m$ nanometer-sized domains and the ferroelectric $R 3 c$ phase in the form of lamellar domains. The remanent ferroelectric $R 3 c$ phase at zero field acted as the seed for the phase transition, significantly reducing the critical field and hence leading to an ultrahigh strain.
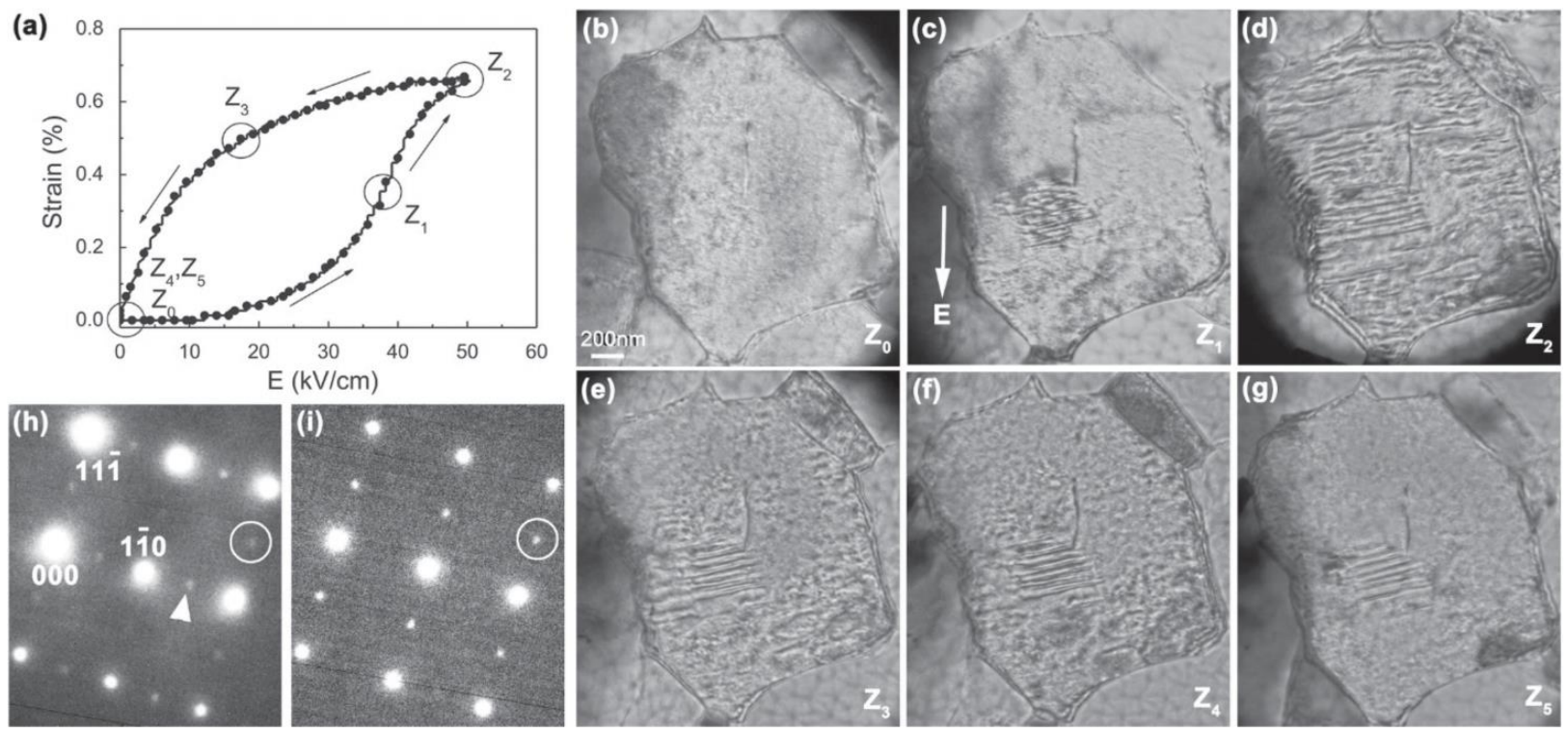

Figure 38. (a) The unipolar strain curve of BNT-2.5Nb ceramic. (b-g) Domain morphology of BNT-2.5Nb ceramics under an electric field marked in (a). (h) and (i) are the SAED patterns of (b) and (d), respectively. The solid triangle and hollow circle indicate the ${ }^{1 / 2}\{o o e\}$ and ${ }^{1 / 2}\{o o o\}$ superlattice diffraction spots, respectively. $\mathrm{Z}_{0}, \mathrm{Z}_{1}, \mathrm{Z}_{2}, \mathrm{Z}_{3}, \mathrm{Z}_{4}$, and $Z_{5}$ represent the points of applying the corresponding electric field in (a). (a-g) Reproduced from ref. 58. with permission from the Wiley-VCH, Copyright 2016. 


\subsection{Nano-domain vs. Core-shell structure}

Differing from BT- and KNN-based ceramics, BNT is prone to forming the submicron/macro coreshell structure with other additives. The representative example is BNT- $x \mathrm{SrTiO}_{3}(\mathrm{BNT}-x \mathrm{ST})$ system. ${ }^{505}$, ${ }^{506}$ Such a core-shell structure exhibited a heterogeneous element distribution and was different from the one observed in BNT-6\%BT ceramics in which the homogeneous element distribution was obtained. ${ }^{491}$ Koruza et al. that revealed the formation of core-shell structure in BNT- $2.5 \%$ ST ceramics was caused by the faster nucleation rate of the BNT phase than that of the ST phase. ${ }^{505}$ Recently, Tan et al. used dual-stimuli in situ TEM to reveal the temperature-dependent electrical properties of BNT$2.5 \% \mathrm{ST}$ ceramics by simultaneously considering the electric field and temperature (see Figure 39 ). ${ }^{506}$ At room temperature (e.g., $25^{\circ} \mathrm{C}$ ), nano-domains changed into the wedge-shape large domains under a bias voltage of $360 \mathrm{~V}$ (see Figure 39(a, b)). These wedge-shaped domains were preserved after removing the bias voltage and even grew wider (see Figure 39(c)). Thus, the ferroelectric phase induced at $25{ }^{\circ} \mathrm{C}$ was metastable, and the ceramics were nonergodic at room temperature. Heating to $41{ }^{\circ} \mathrm{C}$ higher than its $T_{\mathrm{d}} \sim 40{ }^{\circ} \mathrm{C}$, the major large domains disappeared (see Figure $39(\mathrm{~d})$ ). However, a much higher voltage of $420 \mathrm{~V}$ was needed for inducing the large domains, and the large domains exhibited the lamellar shape (see Figure 39(e)). After removing the bias voltage, the large domains completely turned to the nano-domains (see Figure 39(f)). Therefore, such a reversible relaxor-toferroelectric phase transition confirmed the ergodic nature of BNT-2.5\%ST ceramic at $41{ }^{\circ} \mathrm{C}$. The TEM results were highly consistent with the macro electrical properties (see Figure 39(g, h)). Therefore, BNT-2.5\%ST ceramics exhibited the large $P_{\mathrm{r}}$ and $S_{\text {rem }}$ because of the retention of electric-induced ferroelectric domains (e.g., nonergodic behavior) at $25^{\circ} \mathrm{C}$, and the reduced $P_{\mathrm{r}}$ and negligible $S_{\text {rem }}$ at 50 ${ }^{\circ} \mathrm{C}$ were caused by the reversible relaxor-to-ferroelectric phase transition. 

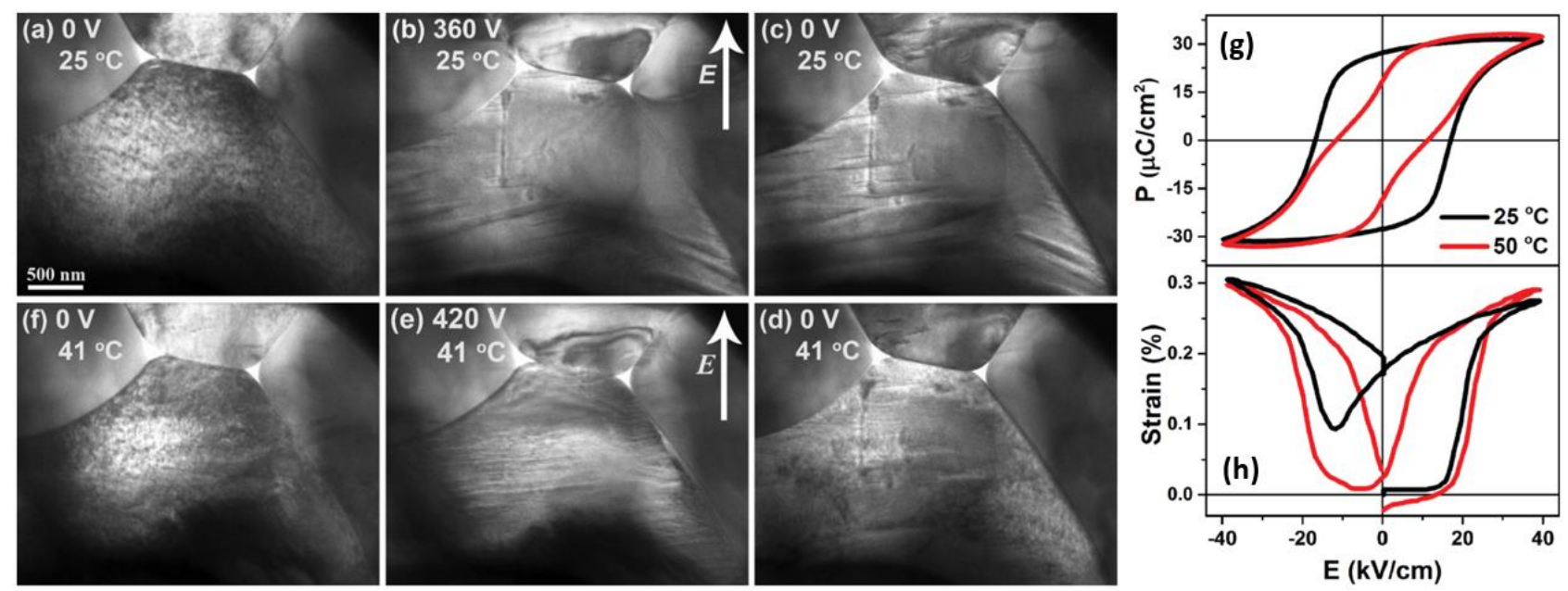

Figure 39. Domain morphology of BNT-2.5\%ST ceramics at $25^{\circ} \mathrm{C}$ with a bias voltage of (a) $0 \mathrm{~V}$, (b) $360 \mathrm{~V}$, and (c) removal of bias voltage; at $41{ }^{\circ} \mathrm{C}$ with a bias voltage of (d) $0 \mathrm{~V}$, (e) $420 \mathrm{~V}$, and (f) removal of bias voltage. (g) $P-E$ loops and (h) $S-E$ curves of BNT-2.5\%ST ceramics at $25{ }^{\circ} \mathrm{C}$ and $50{ }^{\circ} \mathrm{C}$. (a-h) Reproduced from ref. 506. with permission from the American Institute of Physics, Copyright 2019.

\subsection{Nano-domain vs. Composite ceramic}

Thermal depolarization is one of the biggest challenges of BNT-based ceramics for their practical applications. There is an obvious $T_{\mathrm{d}}$ in BNT-based ceramics, over which the macro piezoelectric properties disappear. ${ }^{57}$ To solve this challenge, Zhang et al. proposed a composite ceramic by compositing high-performance BNT-6BT ceramic matrix and polar ZnO particles. ${ }^{57}$ Such a composite ceramic exhibited an increasing $T_{\mathrm{d}}$ with increasing $\mathrm{ZnO}$ content and displayed the disappeared $T_{\mathrm{d}}$ at $30 \mathrm{~mol} \% \mathrm{ZnO}$ (e.g., BNT-6BT: 0.3ZnO). They ascribed the increased and disappeared $T_{\mathrm{d}}$ to the compensated electric field induced by the $\mathrm{ZnO}$ particles. However, further microstructure analysis of ferroelectric domains was still needed. Thus, Tan et al. conducted the systematic in situ temperaturedependent TEM measurements on BNT-6BT: $0.3 \mathrm{ZnO}$ composite ceramic. ${ }^{507}$ The micron-sized domains were observed at BNT-6BT ceramic matrix at $20^{\circ} \mathrm{C}$, and these micron-sized domains 
belonged to an $R 3 c$ phase due to the presence of the ${ }^{1 / 2}\{o o o\}$ superlattice spot (see Figure $\left.40(a)\right)$. The major domain morphology preserved when heating up to $150{ }^{\circ} \mathrm{C}$ (see Figure $40(\mathrm{~b})$ ). But the left edge of the BNT-6BT ceramic matrix started to be occupied by the nano-domains with a $P 4 \mathrm{bm}$ phase (see Figure $40(\mathrm{~b})$ ). The nano-domains completely occupied the left edge at $170{ }^{\circ} \mathrm{C}$ (see Figure $40(\mathrm{c})$ ). As temperature further increased, the micron-sized domains were further replaced by nano-domains (see Figure 40(d-f)). Finally, no micron-sized domains were observed in BNT-6BT ceramic matrix (see Figure 40(g)). Meanwhile, the SAED pattern exhibited no ${ }^{1 / 2}\{o o o\}$ superlattice spots (see Figure 40(g)). In particular, the domains near $\mathrm{ZnO}$ particles disappeared slowest with increasing temperature. Therefore, in situ temperature-dependent TEM measurements demonstrated that there was still a thermal depolarization in BNT-6BT: 0.3ZnO composite ceramic at a temperature more or less the same as BNT-6BT ceramics. That is to say, rather than the increasing or delaying $T_{\mathrm{d}}$, the thermal depolarization of BNT-6BT: 0.3ZnO composite ceramic became gradual and spread over a temperature range spanning approximately $90^{\circ} \mathrm{C}$, much broader than that in BNT-6BT ceramics.
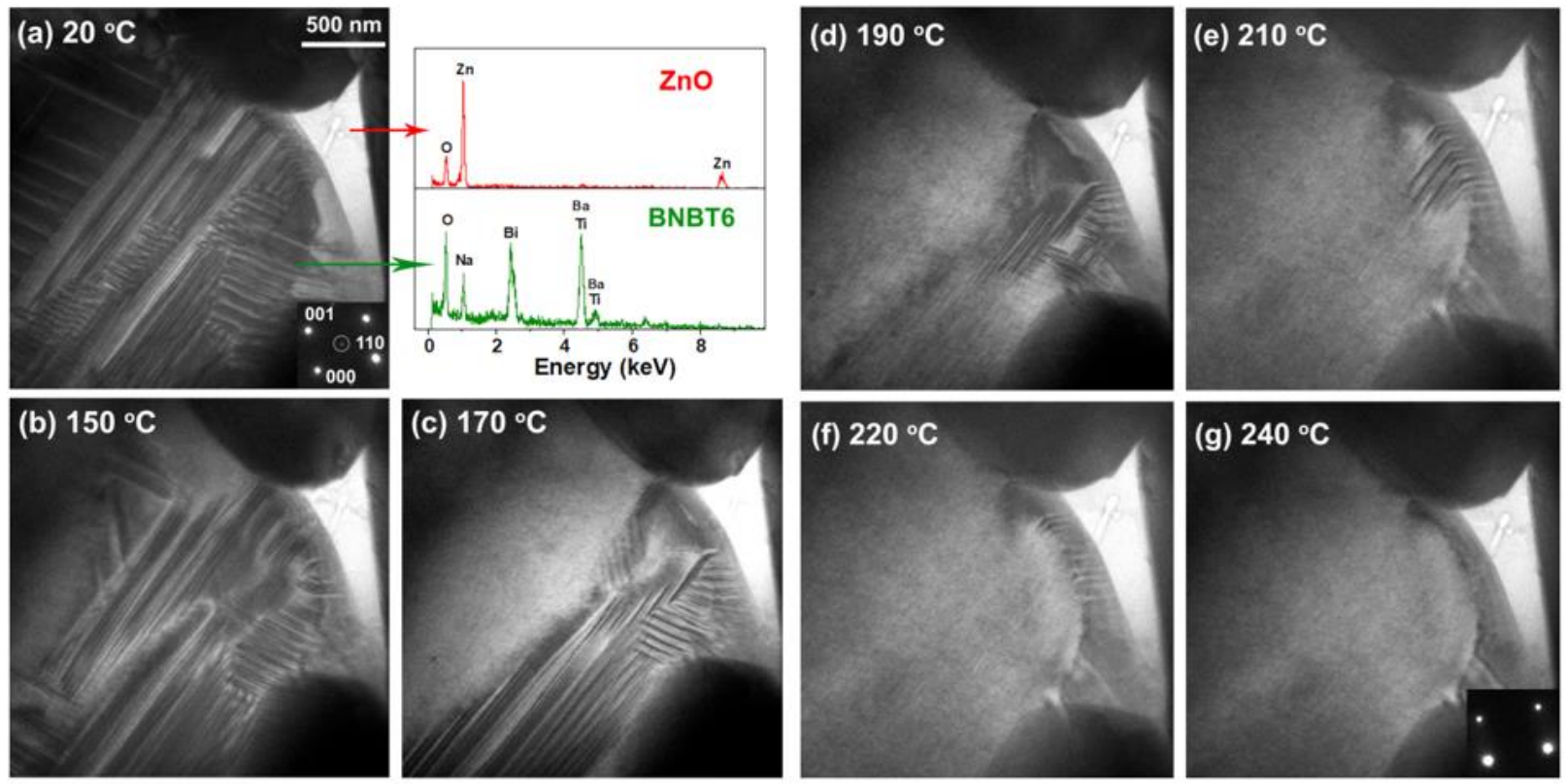

Figure 40. in situ temperature-dependent domain morphology of BNT-6BT: 0.3ZnO composite ceramic at (a) $20{ }^{\circ} \mathrm{C}$,

(b) $150{ }^{\circ} \mathrm{C}$, (c) $170{ }^{\circ} \mathrm{C}$, (d) $190{ }^{\circ} \mathrm{C}$, (e) $210{ }^{\circ} \mathrm{C}$, (f) $220^{\circ} \mathrm{C}$, and (g) $240{ }^{\circ} \mathrm{C}$. The inset of (a) is the energy disperse 
spectroscopy (EDS) of the BNT-6BT ceramic matrix and ZnO particle. (a-g) Reproduced from ref. 507. with permission from the American Physical Society, Copyright 2019.

Considering the slowest fading rate of domains near the $\mathrm{ZnO}$ particle, Tan et al. reasonably proposed the pining effect induced by the $\mathrm{ZnO}$ particle.$^{507} \mathrm{ZnO}$ particle has a spontaneous polarization along the polar $<0001>$ axis. Thus, such a pining effect should be anisotropic. To prove this proposal, they chose a representative $\mathrm{ZnO}$ particle (see Figure 41(a, a1)). Two clusters of micron-sized domains were observed, as marked by the black and red boxes (see Figure $41(\mathrm{a}, \mathrm{a} 2)$ ). At $150{ }^{\circ} \mathrm{C}$, both two clusters of micron-sized domains preserved (see Figure $41(\mathrm{~b})$ ). At $160{ }^{\circ} \mathrm{C}$, only the micron-sized domains inside the red box survived (see Figure 41(c)). These micro-sized domains even survived at $230{ }^{\circ} \mathrm{C}$ and finally disappeared at $300{ }^{\circ} \mathrm{C}$ (see Figure $41(\mathrm{~d}$, e)). Therefore, in situ TEM experiments demonstrated the pining effect of the $\mathrm{ZnO}$ particle. To vividly describe the pining effect, they depicted the schematic illustrations (see Figure 41(f, g)). Due to the existence of spontaneous polarization, the top and bottom of the $\mathrm{ZnO}$ particle along $<0001>$ axis gathered the negative and positive charges, and no charges would gather along the direction perpendicular to $<0001>$ axis. Thus, both two clusters of micronsized domains were preserved at $T<T_{\mathrm{d}}$, and only the micron-sized domains on the bottom edge survived at $T_{\mathrm{d}}<T<T_{\mathrm{m}}$ (see Figure 41(f, g)). Therefore, the in situ TEM measurements provided different but deeper insights into the physical mechanisms of BNT-6BT: 0.3ZnO composite ceramic. 


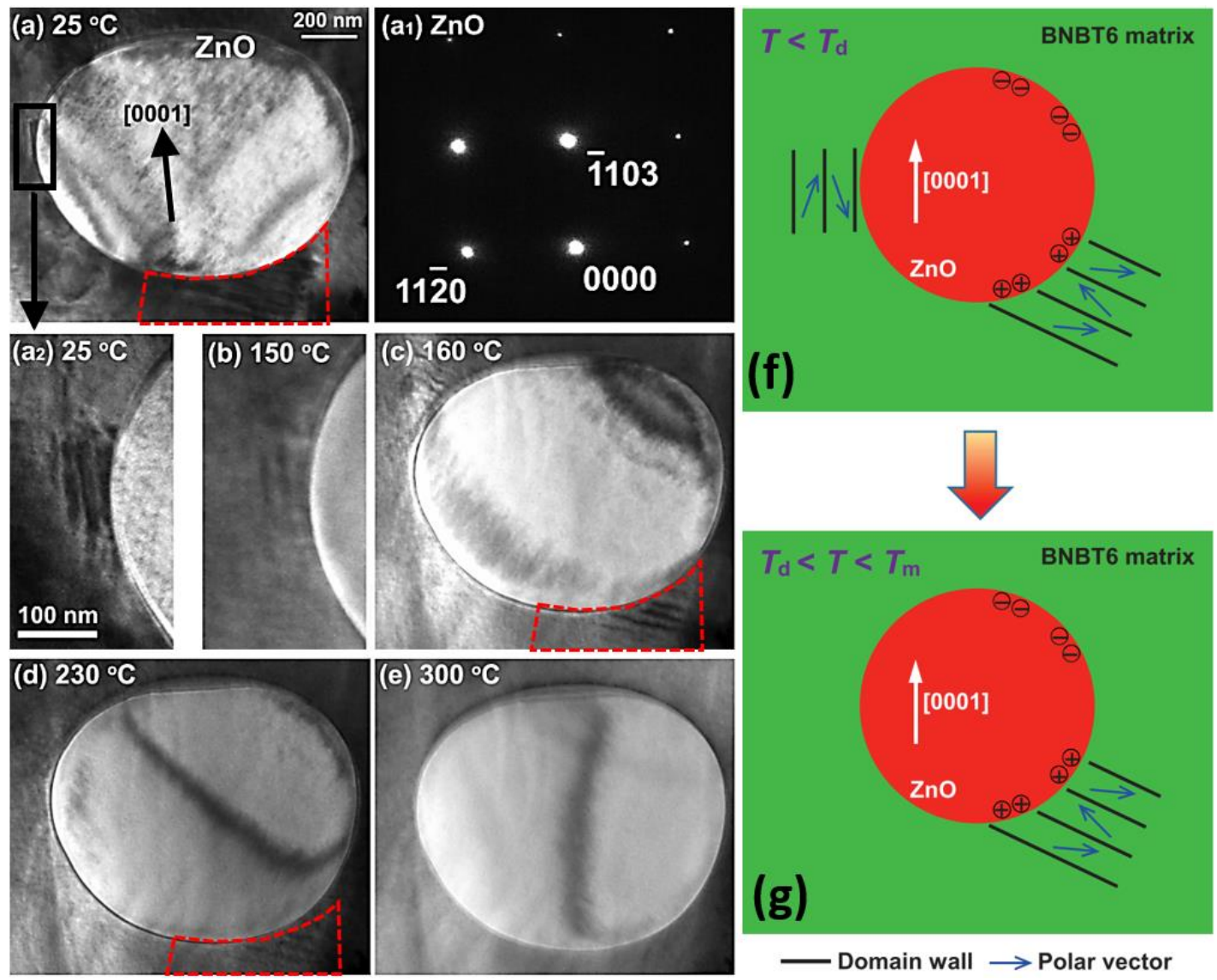

Figure 41. in situ temperature-dependent domain morphology of BNT-6BT: $0.3 \mathrm{ZnO}$ composite ceramic at (a) $25^{\circ} \mathrm{C}$, (b) $150{ }^{\circ} \mathrm{C}$, (c) $160^{\circ} \mathrm{C}$, (d) $230^{\circ} \mathrm{C}$, and (e) $300^{\circ} \mathrm{C}$. (a1) SAED pattern of ZnO particle. (a2) Enlarged view of box area of (a). Schematic illustration of the anisotropic pinning effect from a $\mathrm{ZnO}$ particle on the lamellar ferroelectric domains in the BNT-6BT ceramic matrix. (f) The situation at temperatures below $T_{\mathrm{d}}$; (g) the situation at temperatures between $T_{\mathrm{d}}$ and $T_{\mathrm{m}}$. The polar $<0001>$ direction of $\mathrm{ZnO}$ is marked with the white arrow. (a-g) Reproduced from ref. 507. with permission from the American Physical Society, Copyright 2019.

\subsection{Nano-domain vs. Fatigue}

Fatigue degradation is one of the biggest challenges for ferro/piezoelectric materials' practical application. ${ }^{4,12-15}$ Although extensive efforts were paid on the study of fatigue behavior and the various modes (e.g., passive layer formation, nucleation inhibition, local phase decomposition, near-byelectrode injection, defect redistribution, and domain wall pinning) were proposed to explain the macro 
fatigue degradation, the physical origin of fatigue degradation remains controversial. ${ }^{452,}{ }^{453}$ More importantly, the previously proposed modes were mainly based on the observed macro fatigue degradation, and the actual fatigue degradations are synergistically caused by different factors (models). ${ }^{452,} 453$ The fatigue degradation is closely related to the polarization reversal that is intrinsically linked to ferroelectric domain arrangement and transformation during the electric cycle. Therefore, in situ domain observations during the electric cycles are key to understand the microstructure mechanisms of ferroelectric fatigue degradation. In this context, Tan et al. conducted the in situ TEM measurements on $\left(\left(\mathrm{Bi}_{1 / 2} \mathrm{Na}_{1 / 2}\right)_{0.95} \mathrm{Ba}_{0.05}\right)_{0.98} \mathrm{La}_{0.02} \mathrm{TiO}_{3}$ (BNT52) ceramics during bipolar electric cycles to reveal the physical origin of BNT-based ceramics' fatigue degradation at the nanoscale (see Figure 42). ${ }^{508}$ The unpoled grain of BNT52 ceramics exhibited the nano-domains with coexisting $R 3 \mathrm{c}$ and $P 4 \mathrm{bm}$ phases. Applying an electric field of $30 \mathrm{kV} / \mathrm{cm}$ completely transformed the nano-domains into the large lamellar domains with the $R 3 c$ phase (see Figure $42(\mathrm{a}, \mathrm{b})$ ). Then, the large lamellar domains were gradually disrupted into the small fragments with nano-sized during the increasing electric cycles from 1 to $10^{3}$ (see Figure 42(d-j)). The fatigued grain exhibited the very weak ${ }^{1 / 2}\{$ ooe $\}$-type superlattice diffraction spots (see Figure $42(\mathrm{c})$ ), indicating the existence of the $P 4 b m$ phase. This phenomenon indicated that the bipolar electric cycles not only disrupted the large $R 3 c$ domains but also recovered the $P 4 \mathrm{bm}$ phase, which may be due to the close free energies of those different phases. The nanofragments exhibited the immobilization of the domain wall, which needed a higher electric field (e.g., $40 \mathrm{kV} / \mathrm{cm}$ ) to achieve an effective domain wall motion. 

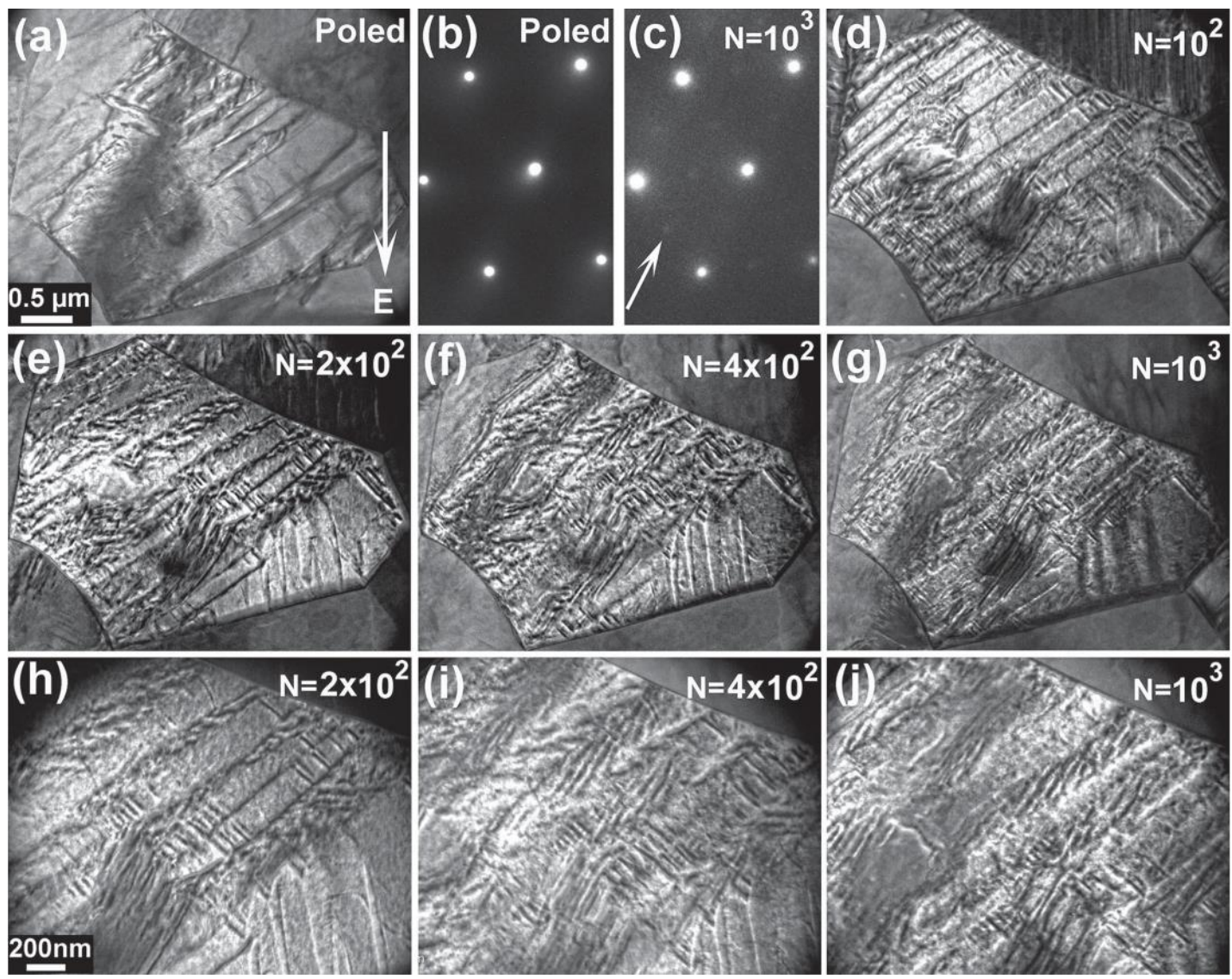

Figure 42. Domain fragmentation of BNT52 ceramics during bipolar electric cycling on a $<111>$-aligned grain. (a)

Domain morphology and (b) the corresponding SAED pattern of the initially poled state at $30 \mathrm{kV} / \mathrm{cm}$ before cycling.

(c) SAED pattern from the same area after $10^{3}$ cycles of bipolar fields. The arrow in (c) suggests the evidence of

${ }^{1 / 2}\{$ ooe $\}$-type superlattice diffraction spots. Domain morphology (d) after $10^{2}$; (e) $2 \times 10^{2}$; (f) $4 \times 10^{2}$; and (g) $10^{3}$ bipolar electric cycles. (h-j) Enlarged images are used to show the nanofragments. The arrow in (a) indicates the positive direction of bipolar electric cycles. (a-j) Reproduced from ref. 508. with permission from the Wiley-VCH, Copyright 2015.

Then, the variations of XRD patterns and electrical properties during the bipolar electric cycles were measured to correlate with the in situ domain observations (see Figure 43). ${ }^{508}$ The $^{1 / 2}\{311\}$ superlattice diffraction peak of the unpoled BNT52 ceramics was too weak to be detected, but the poled BNT52 ceramics displayed an apparent ${ }^{1 / 2}\{311\}$ superlattice diffraction peak because of the irreversible 
electric-induced phase transition from $R 3 c+P 4 b m$ to $R 3 c$ (see Figure 43(a)). With increasing bipolar electric cycles, the intensity of ${ }^{1 / 2}\{311\}$ superlattice diffraction peak slightly increased, and the corresponding full width of half maximum (FWHM) rapidly increased (see Figure 43(b)). The increased FWHM indicated the reduced size of the domains with the $R 3 c$ phase. ${ }^{508}$ Furthermore, the decrease in $S_{\text {neg, }}, S_{\text {pos }}$, maximum polarization $\left(P_{\max }\right)$, and $P_{\mathrm{r}}$ also indicated the degradation of domain reversal and domain wall motion (see Figure 43(c, d)). ${ }^{508}$ The immobilization of the domain wall was supported by the rapid increase in $E_{\mathrm{c}}$ (see Figure 43(d)). Therefore, the macrostructure and the electrical properties correlated well with the in situ domain observations. Generally, the $180^{\circ}$ polarization reversal in rhombohedral ferroelectric perovskites was accomplished through consecutive non- $180^{\circ}$ ferroelastic domain switching instead of the straightforward reverse. ${ }^{508}$ Therefore, the nanosized domain fragments were suggested to result from the frozen intermediate domains for the $180^{\circ}$ polarization reversal. The pinned nanoscale domain fragments led to the reduction in switchable polarization by destructing long-range polar order. ${ }^{508}$ The similar domain fragmentation during electric cycles was also reported in BNT-6BT ceramics. ${ }^{509}$ Simons et al. used the neutron diffraction to detect the phase structure of BNT-6BT ceramics during the bipolar electric fields. ${ }^{509}$ The results indicated the unpoled BNT-6BT ceramic firstly changed into the poled state with long-range ordered domains, and then the domains were progressively fragmented by a repetitive process of domain wall pinning and subdivision. ${ }^{509}$ 

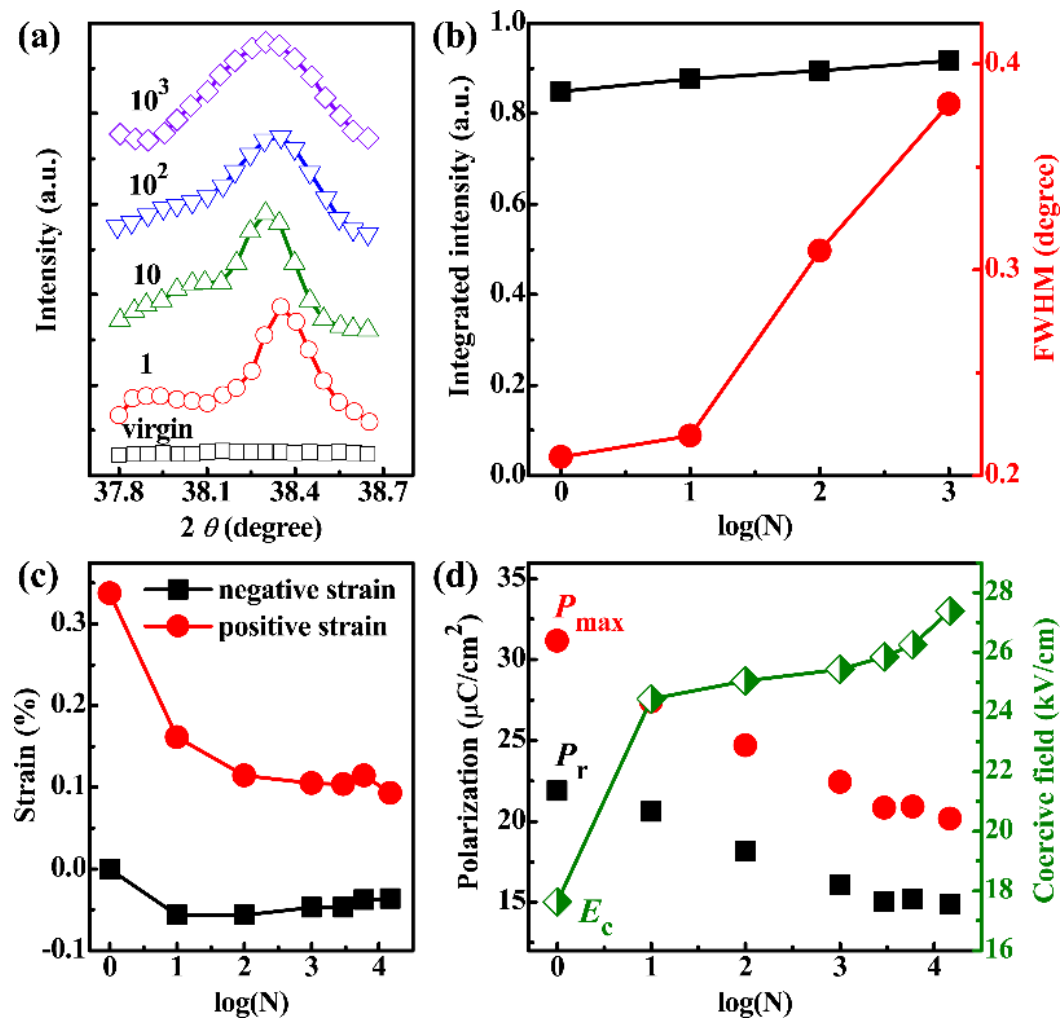

Figure 43. XRD patterns and macro electrical properties of BNT52 ceramics during the bipolar electric cycles. ${ }^{508}$ (a)

$1 / 2\{311\}$ superlattice diffraction peak and (b) its integrated intensity and the full width of half maximum (FWHM).

Variations of (c) $S_{\text {neg }}$ and $S_{\mathrm{pos}}$, and (d) $P_{\max }, P_{\mathrm{r}}$, and $E_{\mathrm{c}}$ during the fatigue measurement. (a-d) Reproduced from ref. 508. with permission from the Wiley-VCH, Copyright 2015.

\section{Summary, challenges, and outlook}

\subsection{Summary}

Based on the above summary and analysis, how the domain structure is affected, and its effects on the performance of lead-free piezoceramics are summarized in Figure 44. The modification of pristine lead-free piezoceramics directly affects the final performance at a macroscopic level. This process is experimental, which means that performance enhancement can be achieved empirically. On the other hand, the modification influences the domain structure, specifically the occurrence of nano-domains and PNRs. Then, these complex domains are stimulated by external stimuli, such as electric field and 
temperature. Through the domain switching, domain wall motion, and domain transformation, the complex domains respond to the external stimulus, resulting in the macro performance we observe eventually. Therefore, the domain structure affects the lead-free piezoceramics' performance at a microstructure/mesoscopic level, which belongs to the theoretical area. In summary, the domain structure acts as a "bridge" connecting the modifications and the macro performance, playing in a crucial role in understanding and achieving high performance.

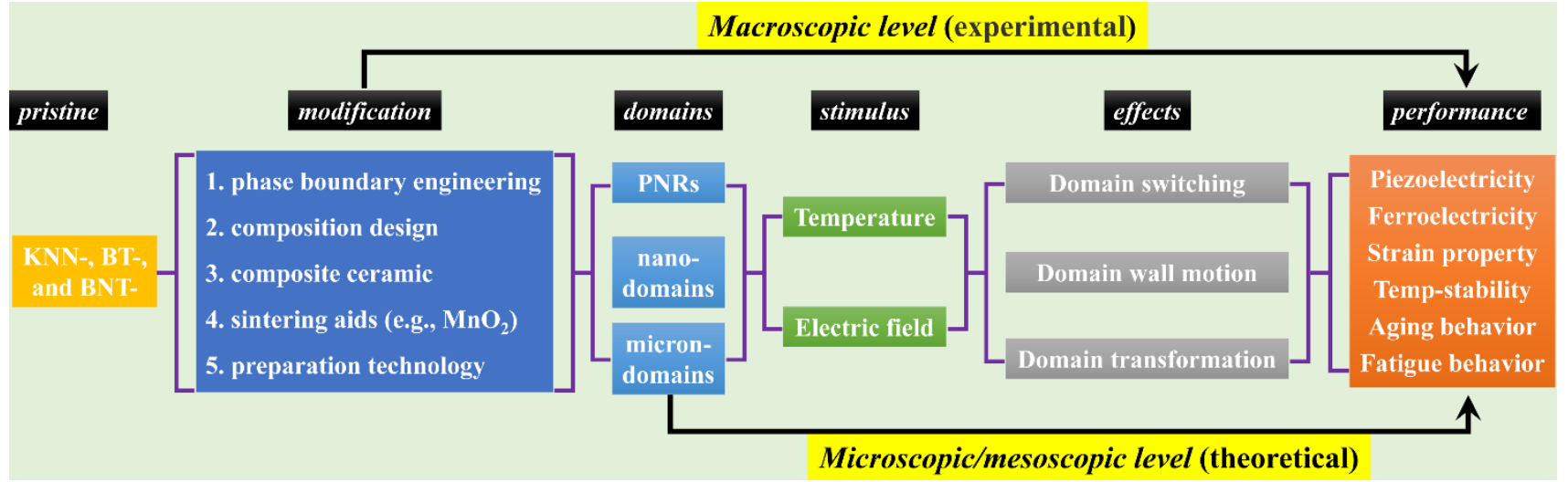

Figure 44. A summary describing how the domain structure is affected, and its effects on the performance of leadfree piezoceramics.

Lead-free piezoelectric ceramics are paid more and more attention because of the promising potential for replacing the lead-based ones. As one of the external contributions, the nano-domains and PNRs play an important role in the macro performance of lead-free piezoelectric ceramics, particularly in improving the piezoelectric properties. For KNN- and BT-based ceramics, we firstly summarized the relationship of phase structure and nano-domains, and then discussed the effects of nano-domains and PNRs on the performance including piezoelectricity, temperature stability, aging, and fatigue behavior. For BNT-based ceramics, nano-domains of different but representative BNT-based material systems (e.g., BNT-BT, BNT-BKT, BNT-BT-KNN, BNT-2.5Nb, BNT-6BT: ZnO, BNT52, and BNT-ST) were reviewed. Specifically, nano-domains varying with composition, temperature, and electric field were 
summarized, focusing on explaining the physical mechanisms for the electrical properties. Finally, how the domain structure was affected, and its effects on the performance of lead-free piezoceramics were also highly comprehensibly summarized. Therefore, this review can promote the understanding and development of lead-free piezoceramics in the future, particularly in nano-domains and PNRs.

\subsection{Challenges}

Although the recent publications have achieved some breakthroughs in observing and analyzing the nano-domains of lead-free piezoelectric ceramics, there are still challenges for further development.

\subsection{1 in situ observation for nano-domains}

Considering the important role of nano-domains in the piezoelectricity enhancement of lead-free piezoceramics, in situ observations for nano-domains under external stimulus (e.g., electric field, temperature, and stress) are key to analyze the underlying physical mechanisms. Although there have already some investigations on the in situ TEM observations for nano-domains, more efforts should be given out due to the significance of explaining the related performance and physical mechanisms. According to the publications reported so far, many efforts were paid on BT- and BNT-based ceramics, but KNN-based ceramics received little attention. This phenomenon may be related to the resource of in situ TEM and the difficulty in fabricating appropriate in situ TEM samples. However, considering the high piezoelectric properties and favorable temperature stability of KNN-based ceramics and the remarkable role of nano-domains in these properties, it is urgent to conduct in situ observations for KNN-based ceramics' nano-domains. 


\subsubsection{Understanding the formation of nano-domains}

Although the recent tools allow us to observe nano-domains, the exact growth mechanism of nanodomains is still absent. Most publications ascribed the formation of nano-domains to the multi-phase coexistence, in which a low domain wall energy was expected due to the low degree of polarization anisotropy energy. ${ }^{59,62-65,158}$ This explanation only proposes the possible reason for the formation of nano-domains from the view of free energy. However, the explanation from the viewpoint of nucleation growth is still missing, which will fundamentally promote the understanding of the formation of nanodomains.

\subsubsection{Phase structure of nano-domain}

Although the phase structure of BNT-based ceramics' nano-domains was extensively studied, the phase structure of BT- and KNN-based ceramics' nano-domains has not well been understood. Most publications on BT- and KNN-based ceramics did not well correlate the nano-domains with their phase structure. This is probably due to the difficulty in detecting the phase structure of a particular nanodomain because the size of some ultra-fined nano-domains is approximate to the highest resolution of TEM. Indeed, our recent work revealed a homogeneous single phase in KNNS-BNKZ-AgSbO$-\mathrm{Fe}_{2} \mathrm{O}_{3}$ ceramics' TEM sample due to the spatial averaging. ${ }^{66}$ However, the local structure of the sample possesses an R-O-T multi-phase coexistence. This phenomenon is recently also reported in $\left(\mathrm{K}_{0.5} \mathrm{Na}_{0.5}\right) \mathrm{NbO}_{3}-x \mathrm{BaTiO}_{3}(x=0.45)$ solid solution, in which the macro symmetry was a cubic phase but the local symmetry was proved to be a rhombohedral-tetragonal (R-T) coexistence phase. ${ }^{366}$ Therefore, the accurate identification of nano-domains' phase structure is still one of the biggest challenges in lead-free piezoceramics. Some more precise and advanced tools should be used to detect the local 
symmetry of these nano-domains, such as Cs-corrected TEM. ${ }^{21}$

\subsection{Outlook}

Despite the challenges, there is a great future for the nano-domains of piezoceramics. Here we gave out the two most important and promising directions for future development.

\subsubsection{Nano-domain engineering vs. Piezoelectricity}

As mentioned above, the occurrence of nano-domains substantially promotes the piezoelectric properties and the temperature stability of piezoceramics. Therefore, better performance is highly excepted if one can effectively manipulate the nano-domains (as called nano-domain engineering). Up to date, several methods are used to achieve the domain engineering, such as composition design, texturing or single crystal, and electric field. ${ }^{18,53,63,150,312-314,510-512}$ Generally, the composition design can guarantee the domain size but cannot control the orientation of domains. However, texturing or single crystal can effectively control the orientation of domains due to the oriented growth, and the further orientation of domains can be achieved by applying an electric field. Therefore, the synergetic effects of these methods on nano-domains hopefully realize the ultra-high performance in lead-free piezoceramics. This conception has been well realized in lead-based piezoelectric materials, such as PMN-PT-Sm ceramic and single crystal. ${ }^{82,129,513}$ In the same way, some researchers also obtained the

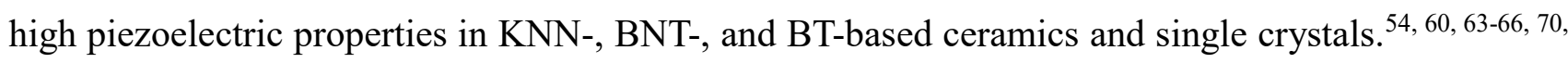
73, 86, 154, 514 However, there is still a distinct gap in the performance between lead-free and lead-based piezoelectric materials. Thus, the further promotion of piezoelectric properties in lead-free ceramics is highly promising when further optimizing the nano-domain engineering. 


\subsubsection{Nano-domain engineering vs. Microdevices}

Besides the promising application in piezoelectric properties, the nano-domain engineering is also highly promising in microdevices. As we know, the ferroelectric domain can be used for the nonvolatile memory (e.g., ferroelectric memory, FRAM). ${ }^{440,515-520}$ The ultra-fine size of nano-domains in lead-free piezoceramics provides several advantages for non-volatile memory, such as small size, high density of domain wall, high response rate and low necessary electric field for driving the polarization reversal. With the microfabrication technology, these advantages endow the lead-free piezoceramics with excellent performance in the ferroelectric memory. Currently, lead-based piezoelectric materials are widely used for FRAM, such as PZT family. ${ }^{440,515-520}$ Therefore, the nano-domain engineering of lead-free piezoceramics has great potential in FRAM when considering the above-mentioned advantages. Furthermore, the high piezoelectric response of nano-domains endows lead-free piezoceramics with the potential in other microdevices. Recently, it was reported that piezoelectric nanogenerators (PENGs) and transducers, consisting of PBE-modified KNN-based ceramics showing nano-domains, exhibited comparable performance to those containing soft lead-based piezoceramics. ${ }^{521}$ In particular, Zhou et al. successfully fabricated a lead-free ultrasonic energy harvester (LF-UEH) by using PBE-modified KNN-based (KNNS-BLNLZ) ceramics. ${ }^{522}$ The LF-UEH possessed good mechanical flexibility and could be driven by the ultrasound to produce adjustable electrical outputs, even in an implanted environment. Therefore, the nano-domain engineering of leadfree piezoceramics has great potential in microdevices. 


\section{Biographies}

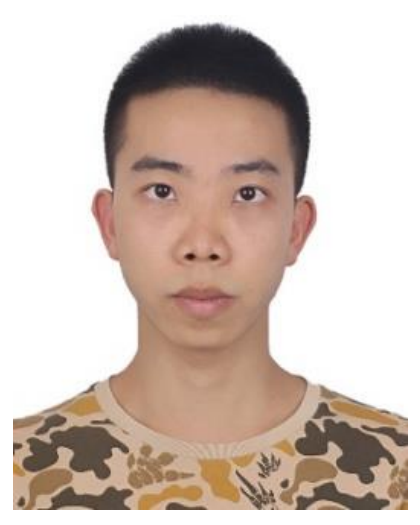

Xiang Lv received his B.S. in Materials Chemistry and Ph.D. degree in Materials Physics and Chemistry from Sichuan University in 2015 and 2019, respectively. He finished his Ph.D. thesis under the guide of Prof. Jiagang Wu. His main research interest is the relationship of "composition designphase/micro structure-performance-physical mechanism" in lead-free piezoelectric ceramics. Currently, he is a postdoctoral fellow at King Abdullah University of Science and Technology.

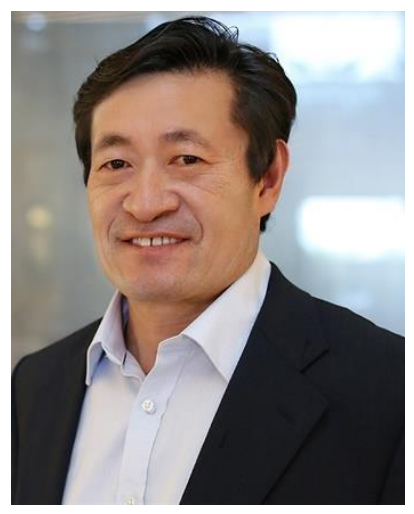

Prof. Xi-xiang Zhang obtained his Ph.D. degree from the University of Barcelona, Spain in 1992. After working as the research scientist at Department de Fisica Fonamental, University de Barcelona for five years (1992-1997), he joined in Hong Kong University of Science and Technology as an assistant professor and then became a full professor in July 2008. In September 2008, he joined KAUST as the manager/director of the core lab and became a full professor in January 2014. His research interests 
include magnetism, spintronics, nano-materials, multiferroic materials, two-dimension (2D) materials and graphene. He is a fellow of the American Physical Society.

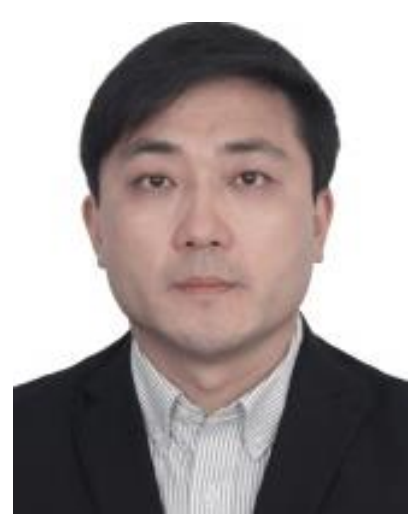

Prof. Jiagang $\mathrm{Wu}$ received his Ph.D. degree in Materials Physics and Chemistry from Sichuan University in 2008. He then joined Prof. John Wang's group (National University of Singapore) as a Singapore Millennium Postdoctoral Fellow (SMF-PDF) from 2008 to 2010. He then started working at Sichuan University in 2011 and became a full professor in 2015. He is the vice-dean of the College of Materials Science and Engineering. His research interest concentrates on the relationship of "structure-composition design-property modification-physical mechanisms" in ferro/piezoelectric/multiferroic materials. He has published more than 160 papers as the first author or corresponding author.

\section{Conflicts of interest}

There are no conflicts of interest to declare.

\section{Acknowledgments}

The authors thank the support from the National Natural Science Foundation of China (NSFC Nos. 
51722208 and 51972215) and the Key Technologies Research and Development Program of Sichuan Province (No. 2018JY0007).

\section{Reference}

1. H. Jaffe, J. Am. Ceram. Soc., 1958, 41, 494-498.

2. C. A. Randall, A. Kelnberger, G. Yang, R. Eitel and T. R. Shrout, J. Electroceram., 2005, 14, 177-191.

3. T. R. Shrout and S. J. Zhang, J. Electroceram., 2007, 19, 113-126.

4. J. Rödel, W. Jo, K. T. Seifert, E. M. Anton, T. Granzow and D. Damjanovic, J. Am. Ceram. Soc., 2009, 92, 1153-1177.

5. B. Jaffe, Piezoelectric ceramics, Elsevier, 2012.

6. J.-F. Li, K. Wang, F.-Y. Zhu, L.-Q. Cheng and F.-Z. Yao, J. Am. Ceram. Soc., 2013, 96, 36773696.

7. P. Panda and B. Sahoo, Ferroelectrics, 2015, 474, 128-143.

8. J. Rödel, K. G. Webber, R. Dittmer, W. Jo, M. Kimura and D. Damjanovic, J. Eur. Ceram. Soc., 2015, 35, 1659-1681.

9. J. Wu, D. Xiao and J. Zhu, Chem. Rev., 2015, 115, 2559-2595.

10. J. Wu, D. Xiao and J. Zhu, J. Mater. Sci: Mater. Electron., 2015, 26, 9297-9308.

11. C.-H. Hong, H.-P. Kim, B.-Y. Choi, H.-S. Han, J. S. Son, C. W. Ahn and W. Jo, J. Materiomics, 2016, 2, 1-24.

12. J. Koruza, A. J. Bell, T. Frömling, K. G. Webber, K. Wang and J. Rödel, J. Materiomics, 2018, 4, 13-26. 
13. J. Wu, Advances in Lead-free Piezoelectric Materials, Springer, 2018.

14. T. Zheng, J. Wu, D. Xiao and J. Zhu, Prog. Mater. Sci., 2018, 98, 552-624.

15. J. Hao, W. Li, J. Zhai and H. Chen, Mater. Sci. Eng., R, 2019, 135, 1-57.

16. S. O. Leontsev and R. E. Eitel, Sci. Technol. Adv. Mater., 2010, 11, 044302.

17. H.-P. Kim, W.-S. Kang, C.-H. Hong, G.-J. Lee, G. Choi, J. Ryu and W. Jo, in Advanced Ceramics for Energy Conversion and Storage, Elsevier, 2020, pp. 157-206.

18. E. Sun and W. Cao, Prog. Mater. Sci., 2014, 65, 124-210.

19. M. Acosta, Strain Mechanisms in Lead-Free Ferroelectrics for Actuators, Springer, 2016.

20. Y. Zhang and J.-F. Li, J. Mater. Chem. C, 2019, 7, 4284-4303.

21. H. Wu, Y. Zhang, J. Wu, J. Wang and S. J. Pennycook, Adv. Funct. Mater., 2019, 29, 1902911.

22. A. J. Bell and O. Deubzer, MRS Bull., 2018, 43, 581-587.

23. D. Damjanovic and G. A. Rossetti, MRS Bull., 2018, 43, 588-594.

24. J. Gao, X. Ke, M. Acosta, J. Glaum and X. Ren, MRS Bull., 2018, 43, 595-599.

25. A. R. Paterson, H. Nagata, X. Tan, J. E. Daniels, M. Hinterstein, R. Ranjan, P. B. Groszewicz, W. Jo and J. L. Jones, MRS Bull., 2018, 43, 600-606.

26. J. Roedel and J.-F. Li, MRS Bull., 2018, 43, 576-580.

27. K. Shibata, R. Wang, T. Tou and J. Koruza, MRS Bull., 2018, 43, 612-616.

28. K. Wang, B. Malič and J. Wu, MRS Bull., 2018, 43, 607-611.

29. S. Troliermckinstry, S. Zhang, A. J. Bell and X. Tan, Annu. Rev. Mater. Res., 2018, 48, 191217.

30. W. D. Callister, Fundamentals of materials science and engineering, Wiley London, 2000.

31. D. Hall, J. Mater. Sci., 2001, 36, 4575-4601. 
32. M. Barsoum and M. Barsoum, Fundamentals of ceramics, CRC press, 2002.

33. E. Cross, Nature, 2004, 432, 24-25.

34. S. Zhang, R. Xia and T. R. Shrout, J. Electroceram., 2007, 19, 251-257.

35. P. Panda, J. Mater. Sci., 2009, 44, 5049-5062.

36. S. Priya and S. Nahm, Lead-free piezoelectrics, Springer Science \& Business Media, 2011.

37. Y. Xu, Ferroelectric materials and their applications, Elsevier, 2013.

38. F. Li, L. Jin, Z. Xu and S. Zhang, Appl. Phys. Rev., 2014, 1, 011103.

39. J. Li, L. Fei and S. Zhang, J. Am. Ceram. Soc., 2014, 97, 1-27.

40. J. Wu, Z. Fan, D. Xiao, J. Zhu and J. Wang, Prog. Mater. Sci., 2016, 84, 335-402.

41. M. Acosta, N. Novak, V. Rojas, S. Patel, R. Vaish, J. Koruza, G. Rossetti Jr and J. Rödel, Appl. Phys. Rev., 2017, 4, 041305.

42. J. Gao, D. Xue, W. Liu, C. Zhou and X. Ren, Actuators, 2017, 6, 24.

43. J. Xing, T. Zheng, J. Wu, D. Xiao and J. Zhu, J. Adv. Dielectr., 2018, 8, 1830003.

44. H.-C. Thong, C. Zhao, Z. Zhou, C.-F. Wu, Y.-X. Liu, Z.-Z. Du, J.-F. Li, W. Gong and K. Wang, Mater. Today, 2019, 29, 37-48.

45. Z.-H. Zhao, Y. Dai and F. Huang, Sustainable Mater. Technol., 2019, 20, e00092.

46. X. Lv, J. Zhu, D. Xiao, X.-x. Zhang and J. Wu, Chem. Soc. Rev., 2020, 49, 671-707.

47. https://ec.europa.eu/environment/waste/rohs_eee/legis_en.htm.

48. Off. J. Eur. Commun., 1989, L398, 19-23.

49. Japan Electronics and Information Technology Industries Association, Standard of Japan Electronics and Information Technology Industries Association 1998.

50. Off. J. Eur. Commun., 2000, L287, 46-50. 
51. Ministry of Information Industry of the People's Republic of China, Industrial Standard of the People's Republic of China, 2006.

52. Off. J. Eur. Union, 2013, L135, 14-18.

53. Y. Saito, H. Takao, T. Tani, T. Nonoyama, K. Takatori, T. Homma, T. Nagaya and M. Nakamura, Nature, 2004, 432, 84-87.

54. W. Liu and X. Ren, Phys. Rev. Lett., 2009, 103, 257602.

55. X. Wang, J. Wu, D. Xiao, J. Zhu, X. Cheng, T. Zheng, B. Zhang, X. Lou and X. Wang, J. Am. Chem. Soc., 2014, 136, 2905-2910.

56. M. H. Lee, D. J. Kim, J. S. Park, S. W. Kim, T. K. Song, M. H. Kim, W. J. Kim, D. Do and I. K. Jeong, Adv. Mater, 2015, 27, 6976-6982.

57. J. Zhang, Z. Pan, F.-F. Guo, W.-C. Liu, H. Ning, Y. Chen, M.-H. Lu, B. Yang, J. Chen and S.T. Zhang, Nat. Commun., 2015, 6, 6615.

58. X. Liu and X. Tan, Adv. Mater, 2016, 28, 574-578.

59. B. Wu, H. Wu, J. Wu, D. Xiao, J. Zhu and S. J. Pennycook, J. Am. Chem. Soc., 2016, 138, 15459-15464.

60. K. Xu, J. Li, X. Lv, J. Wu, X. Zhang, D. Xiao and J. Zhu, Adv. Mater., 2016, 28, 8519-8523.

61. M.-H. Zhang, K. Wang, Y.-J. Du, G. Dai, W. Sun, G. Li, D. Hu, H. C. Thong, C. Zhao, X.-Q. Xi, Z.-X. Yue and J.-F. Li, J. Am. Chem. Soc., 2017, 139, 3889-3895.

62. T. Zheng, H. Wu, Y. Yuan, X. Lv, Q. Li, T. Men, C. Zhao, D. Xiao, J. Wu and K. Wang, Energy Environ. Sci., 2017, 10, 528-537.

63. P. Li, J. Zhai, B. Shen, S. Zhang, X. Li, F. Zhu and X. Zhang, Adv. Mater, 2018, 30, 1705171. 64. Q. Liu, Y. Zhang, J. Gao, Z. Zhou, H. Wang, K. Wang, X. Zhang, L. Li and J.-F. Li, Energy 
Environ. Sci., 2018, 11, 3531-3539.

65. C. Zhao, H. Wu, F. Li, Y. Cai, Y. Zhang, D. Song, J. Wu, X. Lyu, J. Yin and D. Xiao, J. Am. Chem. Soc., 2018, 140, 15252-15260.

66. H. Tao, H. Wu, Y. Liu, Y. Zhang, J. Wu, F. Li, X. Lyu, C. Zhao, D. Xiao and J. Zhu, J. Am. Chem. Soc., 2019, 141, 13987-13994.

67. A. F. Devonshire, Phil. Mag., 1949, 40, 1040-1063.

68. A. F. Devonshire, The London, Edinburgh, and Dublin Philosophical Magazine and Journal of Science, 1951, 42, 1065-1079.

69. L. Egerton and D. M. Dillon, J. Am. Ceram. Soc., 1959, 42, 438-442.

70. Y. Yao, C. Zhou, D. Lv, D. Wang, H. Wu, Y. Yang and X. Ren, EPL (Europhys. Lett.), 2012, 98, 27008.

71. Q. Liu, Y. Zhang, J. Gao, Z. Zhou, D. Yang, K.-Y. Lee, A. Studer, M. Hinterstein, K. Wang, X. Zhang, L. Li and J.-F. Li, Natl. Sci. Rev., 2020, 7, 355-365.

72. T. Zheng, Y. Zhang, Q. Ke, H. Wu, L. W. Heng, D. Xiao, J. Zhu, S. J. Pennycook, K. Yao and J. Wu, Nano Energy, 2020, 70, 104559.

73. Y. Liu, Y. Chang, F. Li, B. Yang, Y. Sun, J. Wu, S. Zhang, R. Wang and W. Cao, ACS Appl. Mater. Interfaces, 2017, 9, 29863-29871.

74. D. Damjanovic, Rep. Prog. Phys., 1998, 61, 1267.

75. D. Damjanovic, IEEE Trans. Ultrason. Ferroelectr. Freq. Control, 2009, 56, 1574-1585.

76. D. Damjanovic, J. Am. Ceram. Soc., 2005, 88, 2663-2676.

77. L. Fan, J. Chen, Y. Ren, Z. Pan, L. Zhang and X. Xing, Phys. Rev. Lett., 2016, 116, 027601.

78. F. Li, S. Zhang, T. Yang, Z. Xu, N. Zhang, G. Liu, J. Wang, J. Wang, Z. Cheng and Z. G. Ye, 
Nat. Commun., 2016, 7, 13807.

79. H. Takenaka, I. Grinberg, S. Liu and A. M. Rappe, Nature, 2017, 546, 391-395.

80. F. Li, D. Lin, Z. Chen, Z. Cheng, J. Wang, C. Li, Z. Xu, Q. Huang, X. Liao and L. Q. Chen, Nat. Mater., 2018, 17, 349-354.

81. B. Narayan, J. S. Malhotra, R. Pandey, K. Yaddanapudi, P. Nukala, B. Dkhil, A. Senyshyn and R. Ranjan, Nat. Mater., 2018, 17, 427-431.

82. F. Li, M. J. Cabral, B. Xu, Z. Cheng, E. C. Dickey, J. M. LeBeau, J. Wang, J. Luo, S. Taylor, W. Hackenberger, L. Bellaiche, Z. Xu, L. Q. Chen, T. R. Shrout and S. Zhang, Science, 2019, 364, 264-268.

83. D. Wang, K. Zhu, H. Ji and J. Qiu, Ferroelectrics, 2009, 392, 120-126.

84. J. Fang, X. Wang, Z. Tian, C. Zhong, L. Li and R. Zuo, J. Am. Ceram. Soc., 2010, 93, 35523555.

85. T. Zheng and J. Wu, J. Mater. Chem. A, 2015, 3, 6772-6780.

86. M. Jiang, J. Zhang, G.-H. Rao, D. Li, C. A. Randall, T. Li, B. Peng, L. Li, Z. Gu and X. Liu, J. Mater. Chem. C, 2019, 7, 14845-14854.

87. D. Liu, X. Zhang, W. Su, X. Wang, W. Yao, C. Zhou and J. Zhang, J. Alloys. Compd., 2019, 779, 800-804.

88. W. Yao, J. Zhang, X. Wang, C. Zhou, X. Sun and J. Zhan, J. Eur. Ceram. Soc., 2019, 39, 287294.

89. F. Gao, R.-Z. Hong, J.-J. Liu, Y.-H. Yao and C.-S. Tian, J. Eur. Ceram. Soc., 2008, 28, $2063-$ 2070.

90. P. Li, X. Chen, F. Wang, B. Shen, J. Zhai, S. Zhang and Z. Zhou, ACS Appl. Mater. Interfaces, 
2018, 10, 28772-28779.

91. Y. Quan, W. Ren, G. Niu, L. Wang, J. Zhao, N. Zhang, M. Liu, Z.-G. Ye, L. Liu and T. Karaki, ACS Appl. Mater. Interfaces, 2018, 10, 10220-10226.

92. H. Zhang, Y. Zhu, P. Fan, M. A. Marwat, W. Ma, K. Liu, H. Liu, B. Xie, K. Wang and J. Koruza, Acta Mater., 2018, 156, 389-398.

93. P. Li, Y. Huan, W. Yang, F. Zhu, X. Li, X. Zhang, B. Shen and J. Zhai, Acta Mater, 2019, 165, 486-495.

94. R. Wang, R. Xie, T. Sekiya and Y. Shimojo, Mater. Res. Bull., 2004, 39, 1709-1715.

95. H. Takahashi, Y. Numamoto, J. Tani and S. Tsurekawa, Jpn. J. Appl. Phys., 2006, 45, 7405.

96. X.-M. Chen, Y.-L. Su, H.-L. Lian, J.-B. Lu, J.-P. Zhou and P. Liu, J. Mater. Sci., 2019, 54, 13457-13466.

97. H. Du, F. Tang, F. Luo, W. Zhou, S. Qu and Z. Pei, Mater. Sci. Eng B, 2007, 137, 175-179.

98. F. Rubio-Marcos, J. J. Romero, D. A. Ochoa, J. E. García, R. Perez and J. F. Fernandez, J. Am. Ceram. Soc., 2010, 93, 318-321.

99. J. Wu, Y. Wang and H. Wang, RSC Adv., 2014, 4, 64835-64842.

100. F.-Z. Yao, K. Wang, W. Jo, J.-S. Lee and J.-F. Li, J. Appl. Phys., 2014, 116, 114102.

101. F. Benabdallah, C. Elissalde, U.-C. C. Seu, D. Michau, A. Poulon-Quintin, M. Gayot, P. Garreta, H. Khemakhem and M. Maglione, J. Eur. Ceram. Soc., 2015, 35, 4153-4161.

102. J. P. Praveen, T. Karthik, A. James, E. Chandrakala, S. Asthana and D. Das, J. Eur. Ceram. Soc., $2015,35,1785-1798$.

103. D. Song, M. S. Woo, J. H. Ahn and T. H. Sung, J. Korean Phys. Soc., 2015, 66, 1549-1553.

104. T. Zheng and J. Wu, ACS Appl. Mater. Interfaces, 2016, 8, 9242-9246. 
105. H. Tao and J. Wu, J. Mater. Chem. C, 2017, 5, 1601-1606.

106. C. Qiu, J. Liu, F. Li and Z. Xu, J. Appl. Phys., 2019, 125, 014102.

107. K. Chen, J. Zhou, F. Zhang, X. Zhang, C. Li and L. An, J. Am. Ceram. Soc., 2015, 98, 16981701.

108. H. Tian, C. Hu, X. Meng, P. Tan, Z. Zhou, J. Li and B. Yang, Cryst. Growth. Des., 2015, 15, $1180-1185$.

109. H. Liu, P. Veber, J. Rödel, D. Rytz, P. B. Fabritchnyi, M. I. Afanasov, E. A. Patterson, T. Frömling, M. Maglione and J. Koruza, Acta Mater., 2018, 148, 499-507.

110. H. Liu, H. Huang, L. Fan, Y. Ren, H. Zhou, L.-Q. Chen, J. Chen and X. Xing, Phys. Rev. Mater, 2018, 2, 111403.

111. L. Fan, J. Chen, Y. Ren and X. Xing, Inorg. Chem., 2018, 57, 3002-3007.

112. S. W. Choi, J. M. Jung and A. S. Bhalla, Ferroelectrics, 1996, 189, 27-38.

113. B. Noheda, D. E. Cox, G. Shirane, R. Guo, B. Jones and L. E. Cross, Phys. Rev. B, 2000, 63, 014103.

114. L. Bellaiche, A. García and D. Vanderbilt, Phys. Rev. B, 2001, 64, 060103.

115. B. Noheda, D. E. Cox, G. Shirane, S. E. Park, L. E. Cross and Z. Zhong, Phys. Rev. Lett., 2001, 86, 3891-3894.

116. Z. G. Ye, B. Noheda, M. Dong, D. Cox and G. Shirane, Phys. Rev. B, 2001, 64, 184114.

117. B. Noheda, D. E. Cox, G. Shirane, J. Gao and Z. G. Ye, Phys. Rev. B, 2002, 66, 054104.

118. B. Noheda, Z. Zhong, D. E. Cox, G. Shirane, S. E. Park and P. Rehrig, Phys. Rev. B, 2002, 65, 224101.

119. W. Dmowski, S. B. Vakhrushev, I. K. Jeong, M. P. Hehlen, F. Trouw and T. Egami, Phys. Rev. 
Lett., 2008, 100, 137602.

120. M. Hinterstein, J. Rouquette, J. Haines, P. Papet, M. Knapp, J. Glaum and H. Fuess, Phys. Rev. Lett., 2011, 107, 077602.

121. M. Hinterstein, M. Hoelzel, J. Rouquette, J. Haines, J. Glaum, H. Kungl and M. Hoffman, Acta Mater., 2015, 94, 319-327.

122. H. Liu, J. Chen, L. Fan, Y. Ren, L. Hu, F. Guo, J. Deng and X. Xing, Chem. Mater, 2017, 29, $5767-5771$.

123. H. Liu, J. Chen, L. Fan, Y. Ren, Z. Pan, K. V. Lalitha, J. Rodel and X. Xing, Phys. Rev. Lett., 2017, 119, 017601.

124. A. Manjón-Sanz, C. M. Culbertson, D. Hou, J. L. Jones and M. R. Dolgos, Acta Mater., 2019, 171, 79-91.

125. H. Liu, J. Chen, H. Huang, L. Fan, Y. Ren, Z. Pan, J. Deng, L. Q. Chen and X. Xing, Phys. Rev. Lett., 2018, 120, 055501.

126. M. Hinterstein, K. Y. Lee, S. Esslinger, J. Glaum, A. J. Studer, M. Hoffman and M. J. Hoffmann, Phys. Rev. B, 2019, 99, 174107.

127. A. Pramanick, W. Dmowski, T. Egami, A. S. Budisuharto, F. Weyland, N. Novak, A. D. Christianson, J. M. Borreguero, D. L. Abernathy and M. R. V. Jorgensen, Phys. Rev. Lett., 2018, 120, 207603.

128. H. Zhang, X. Lu, C. Wang, L. Zheng, B. Yang and W. Cao, Phys. Rev. B, 2018, 97, 054114.

129. F. Li, S. Zhang, Z. Xu, X. Wei and T. R. Shrout, Adv. Funct. Mater., 2011, 21, 2118-2128.

130. J. Hao, W. Bai, W. Li and J. Zhai, J. Am. Ceram. Soc., 2012, 95, 1998-2006.

131. J. Zhang, Y. Qin, Y. Gao, W. Yao and M. Zhao, J. Am. Ceram. Soc., 2014, 97, 759-764. 
132. D. K. Khatua, T. Mehrotra, A. Mishra, B. Majumdar, A. Senyshyn and R. Ranjan, Acta Mater., 2017, 134, 177-187.

133. Z. Cai, X. Wang, W. Hong, B. Luo, Q. Zhao and L. Li, J. Am. Ceram. Soc., 2018, 101, 54875496.

134. C. Kittel, Phys. Rev., 1946, 70, 965.

135. P. G. Lucuta, J. Am. Ceram. Soc., 1989, 72, 933-937.

136. G. Arlt, J. Mater. Sci., 1990, 25, 2655-2666.

137. D. You, W. Jung, S. Choi and Y. Cho, Appl. Phys. Lett., 2004, 84, 3346-3348.

138. H. Yu, H. Zeng, R. Chu, G. Li, H. Luo, Z. Xu and Q. Yin, J. Phys. D: Appl. Phys., 2004, 37, 2914.

139. J. Li, R. Rogan, E. Üstündag and K. Bhattacharya, Nat. Mater., 2005, 4, 776-781.

140. H. Wang, J. Zhu, N. Lu, A. Bokov, Z.-G. Ye and X. Zhang, Appl. Phys. Lett., 2006, 89, 042908.

141. K. A. Schönau, L. A. Schmitt, M. Knapp, H. Fuess, R.-A. Eichel, H. Kungl and M. J. Hoffmann, Phys. Rev. B, 2007, 75, 184117.

142. R. Theissmann, L. A. Schmitt, J. Kling, R. Schierholz, K. A. Schönau, H. Fuess, M. Knapp, H. Kungl and M. J. Hoffmann, J. Appl. Phys., 2007, 102, 024111.

143. H. Wang, J. Zhu, X. W. Zhang, Y. X. Tang and H. S. Luo, J. Am. Ceram. Soc., 2008, 91, 23822384.

144. P. Marton, I. Rychetsky and J. Hlinka, Phys. Rev. B, 2010, 81, 144125.

145. J. Yao, W. Ge, L. Luo, J. Li, D. Viehland and H. Luo, Appl. Phys. Lett., 2010, 96, 222905.

146. X. Lv, J. Wu, S. Yang, D. Xiao and J. Zhu, ACS Appl. Mater. Interfaces, 2016, 8, 18943-18953.

147. F. Li, S. Zhang, Z. Xu and L. Q. Chen, Adv. Funct. Mater., 2017, 27, 1700310. 
148. K. Chen and L. Li, Adv. Mater., 2019, 31, 1901115.

149. F. Li, L. Wang, L. Jin, D. Lin, J. Li, Z. Li, Z. Xu and S. Zhang, IEEE Trans. Ultrason. Ferroelectr. Freq. Control, 2015, 62, 18-32.

150. L. A. Schmitt, K. A. Schönau, R. Theissmann, H. Fuess, H. Kungl and M. J. Hoffmann, J. Appl. Phys., 2007, 101, 074107.

151. J. Fu, R. Zuo and Z. Xu, Appl. Phys. Lett., 2011, 99, 062901.

152. R. López-Juárez, O. Novelo-Peralta, F. González-García and F. Rubio-Marcos, J. Eur. Ceram. Soc., 2011, 31, 1861-1864.

153. Y. Sato, T. Hirayama and Y. Ikuhara, Phys. Rev. Lett., 2011, 107, 187601.

154. C. Hu, X. Meng, M.-H. Zhang, H. Tian, J. E. Daniels, P. Tan, F. Huang, L. Li, K. Wang, J.-F. Li, Q. Lu, W. Cao and Z. Zhou, Sci. Adv., 2020, 6, eaay5979.

155. G. Arlt and P. Sasko, J. Appl. Phys., 1980, 51, 4956-4960.

156. Y. Qin, J. Zhang, Y. Gao, Y. Tan and C. Wang, J. Appl. Phys., 2013, 113, 204107.

157. X. Lv, J. Li, T.-L. Men, J. Wu, X.-x. Zhang, K. Wang, J.-F. Li, D. Xiao and J. Zhu, ACS Appl. Mater. Interfaces, 2018, 10, 30566-30573.

158. X. Sun, J. Zhang, X. Lv, Y. Liu, F. Li and J. Wu, J. Mater. Chem. A, 2019, 7, 16803-16811.

159. Y. Feng, J. Wu, Q. Chi, W. Li, Y. Yu and W. Fei, Chem. Rev., 2020, 120, 1710-1787.

160. Y. Ding, T. Zheng, C. Zhao and J. Wu, J. Appl. Phys., 2019, 126, 124101.

161. D. Ghosh, A. Sakata, J. Carter, P. A. Thomas, H. Han, J. C. Nino and J. L. Jones, Adv. Funct. Mater., 2014, 24, 885-896.

162. Y. Tan, J. Zhang, Y. Wu, C. Wang, V. Koval, B. Shi, H. Ye, R. McKinnon, G. Viola and H. Yan, Sci. Rep., 2015, 5, 9953. 
163. M. Otonicar, S. D. Skapin and B. Jancar, IEEE Trans. Ultrason. Ferroelectr. Freq. Control, 2011, 58, 1928-1938.

164. V. Dorcet and G. Trolliard, Acta Mater, 2008, 56, 1753-1761.

165. I. Levin and I. M. Reaney, Adv. Funct. Mater., 2012, 22, 3445-3452.

166. Y. Qin, S. Zhang, Y. Wu, C. Lu and J. Zhang, J. Eur. Ceram. Soc., 2017, 37, 3493-3500.

167. Y. Chen, H. Ye, X. Wang, Y. Li and X. Yao, J. Eur. Ceram. Soc., 2020, 40, 391-400.

168. C. A. Randall, N. Kim, J. P. Kucera, W. Cao and T. R. Shrout, J. Am. Ceram. Soc., 1998, 81, 677-688.

169. W. Cao and C. A. Randall, J. Phys. Chem. Solids, 1996, 57, 1499-1505.

170. V. Bobnar, Z. Kutnjak and A. Levstik, Appl. Phys. Lett., 2000, 76, 2773-2775.

171. Y. Nakata, Y. Tsujimi, K. Katsuraya, M. Iwata and T. Yagi, Appl. Phys. Lett., 2006, 89, 022903.

172. V. Bovtun, S. Kamba, A. Pashkin, M. Savinov, P. Samoukhina, J. Petzelt, I. P. Bykov and M.

D. Glinchuk, Ferroelectrics, 2004, 298, 23-30.

173. L. E. Cross, Ferroelectrics, 1987, 76, 241-267.

174. L. E. Cross, Ferroelectrics, 1994, 151, 305-320.

175. L. E. Cross, Ferroelectrics, 2008, 76, 241-267.

176. D. Viehland, M. Wuttig and L. Cross, Ferroelectrics, 1991, 120, 71-77.

177. J. Hlinka, J. Adv. Dielectr., 2012, 2, 1241006.

178. M. A. Hentati, H. Dammak, H. Khemakhem, N. Guiblin and M. P. Thi, J. Appl. Phys., 2015, 118, 034104.

179. M. Pavel, I. Rychetský and J. Petzelt, J. Appl. Phys., 2001, 89, 5036-5039.

180. Y. Shi and A. Soh, J. Appl. Phys., 2011, 110, 124108. 
181. A. Bokov and Z.-G. Ye, J. Mater. Sci., 2006, 41, 31-52.

182. I.-W. Chen, L. Ping and W. Ying, J. Phys. Chem. Solids, 1996, 57, 1525-1536.

183. E. Colla, E. Y. Koroleva, N. Okuneva and S. Vakhrushev, J. Phys.: Condens. Matter, 1992, 4, 3671.

184. N. De Mathan, E. Husson, G. Calvarn, J. Gavarri, A. Hewat and A. Morell, J. Phys.: Condens. Matter, 1991, 3, 8159.

185. S. Kamba, M. Kempa, V. Bovtun, J. Petzelt, K. Brinkman and N. Setter, J. Phys.: Condens. Matter, 2005, 17, 3965.

186. C. W. Ahn, C.-H. Hong, B.-Y. Choi, H.-P. Kim, H.-S. Han, Y. Hwang, W. Jo, K. Wang, J.-F. Li and J.-S. Lee, J. Korean Phys. Soc., 2016, 68, 1481-1494.

187. F. Li, Z. Xu and S. Zhang, Appl. Phys. Lett., 2014, 105, 122904.

188. B. Malic and T. Rojac, Nat. Mater., 2018, 17, 297-298.

189. G. Xu, J. Wen, C. Stock and P. Gehring, Nat. Mater., 2008, 7, 562-566.

190. G. Xu, Z. Zhong, Y. Bing, Z.-G. Ye and G. Shirane, Nat. Mater., 2006, 5, 134-140.

191. J. Carreaud, P. Gemeiner, J. Kiat, B. Dkhil, C. Bogicevic, T. Rojac and B. Malic, Phys. Rev. B, 2005, 72, 174115.

192. E. Dul'kin, M. Roth, P.-E. Janolin and B. Dkhil, Phys. Rev. B, 2006, 73, 012102.

193. I. Franke, K. Roleder, L. Mitoseriu, R. Piticescu and Z. Ujma, Phys. Rev. B, 2006, 73, 144114.

194. K. Hirota, Z.-G. Ye, S. Wakimoto, P. Gehring and G. Shirane, Phys. Rev. B, 2002, 65, 104105.

195. J. Macutkevic, J. Banys, A. Bussmann-Holder and A. Bishop, Phys. Rev. B, 2011, 83, 184301.

196. R. Pirc and R. Blinc, Phys. Rev. B, 2007, 76, 020101.

197. R. Pirc, R. Blinc and V. Vikhnin, Phys. Rev. B, 2004, 69, 212105. 
198. S. Wakimoto, C. Stock, Z.-G. Ye, W. Chen, P. Gehring and G. Shirane, Phys. Rev. B, 2002, 66, 224102.

199. G. Xu, P. Gehring and G. Shirane, Phys. Rev. B, 2005, 72, 214106.

200. M. Roth, E. Mojaev, E. Dul'kin, P. Gemeiner and B. Dkhil, Phys. Rev. Lett., 2007, 98, 265701.

201. M. E. Manley, J. W. Lynn, D. Abernathy, E. Specht, O. Delaire, A. Bishop, R. Sahul and J. Budai, Nat. Commun., 2014, 5, 3683.

202. M. E. Manley, D. L. Abernathy, R. Sahul, D. E. Parshall, J. W. Lynn, A. D. Christianson, P. J.

Stonaha, E. D. Specht and J. D. Budai, Sci. Adv., 2016, 2, e1501814.

203. M. Vögler, N. Novak, F. Schader and J. Rödel, Phys. Rev. B, 2017, 95, 024104.

204. J. Banys, J. Macutkevic, R. Grigalaitis and W. Kleemann, Phys. Rev. B, 2005, 72, 024106.

205. N. Liu, R. Dittmer, R. W. Stark and C. Dietz, Nanoscale, 2015, 7, 11787-11796.

206. V. V. Shvartsman and D. C. Lupascu, J. Am. Ceram. Soc., 2012, 95, 1-26.

207. W. Liang, W. Wu, D. Xiao and J. Zhu, J. Am. Ceram. Soc., 2011, 94, 4317-4322.

208. C. Ang, Z. Jing and Z. Yu, J. Phys.: Condens. Matter, 2002, 14, 8901.

209. Y. Guo, K.-i. Kakimoto and H. Ohsato, J. Phys. Chem. Solids, 2004, 65, 1831-1835.

210. M. Kosec, V. Bobnar, M. Hrovat, J. Bernard, B. Malic and J. Holc, J. Mater. Res., 2004, 19, 1849-1854.

211. Z. Yu, C. Ang, R. Guo and A. Bhalla, J. Appl. Phys., 2002, 92, 2655-2657.

212. C.-S. Chen, P.-Y. Chen and C.-S. Tu, J. Appl. Phys., 2014, 115, 014105.

213. V. Bobnar, B. Malič, J. Holc, M. Kosec, R. Steinhausen and H. Beige, J. Appl. Phys., 2005, 98, 024113.

214. V. Bobnar, J. Holc, M. Hrovat and M. Kosec, J. Appl. Phys., 2007, 101, 074103. 
215. Y. Liu, R. Withers, B. Nguyen and K. Elliott, Appl. Phys. Lett., 2007, 91, 152907.

216. J. Yin, C. Zhao, Y. Zhang and J. Wu, Acta Mater., 2018, 147, 70-77.

217. M. Frey and D. Payne, Phys. Rev. B, 1996, 54, 3158.

218. S. Zhang, H. J. Lee, C. Ma and X. Tan, J. Am. Ceram. Soc., 2011, 94, 3659-3665.

219. J. H. Cho, N. R. Yeom, S. J. Kwon, Y. J. Lee, Y. H. Jeong, M. P. Chun, J. H. Nam, J. H. Paik and B. I. Kim, J. Appl. Phys., 2012, 112, 052005.

220. E. Soergel, Appl. Phys. B, 2005, 81, 729-751.

221. Y. Qin, J. Zhang, Y. Tan, W. Yao, C. Wang and S. Zhang, J. Eur. Ceram. Soc., 2014, 34, 41774184.

222. J. Zhang, Y. Gao, Y. Qin, W. Yao and X. Tian, J. Appl. Phys., 2014, 116, 104106.

223. C. Zhou, J. Zhang, W. Yao, X. Wang, D. Liu and X. Sun, J. Appl. Phys., 2018, 124, 164101.

224. M.-H. Zhang, H. C. Thong, Y. X. Lu, W. Sun, J.-F. Li and K. Wang, J. Korean Ceram. Soc., 2017, 54, 261-271.

225. A. Gruverman, M. Alexe and D. Meier, Nat. Commun., 2019, 10, 1661.

226. H. Schmid, Polarized light microscopy (PLM) of ferroelectric and ferroelastic domains in transmitted and reflected lights, Springer, 1993.

227. X. Li, Y. Wang, L. Liu, X. Zhao, H. Luo and D. Lin, Mater. Chem. Phys., 2010, 122, 350-353.

228. F. Rubio-Marcos, A. Del Campo, P. Marchet and J. F. Fernández, Nat. Commun., 2015, 6, 6594.

229. F. Rubio-Marcos, D. A. Ochoa, A. Del Campo, M. A. García, G. R. Castro, J. F. Fernández and J. E. García, Nat. Photonics, 2018, 12, 29-32.

230. X. Lv, J. Wu, D. Xiao, J. Zhu and X. Zhang, Acta Mater., 2017, 140, 79-86.

231. X. Lv, J. Wu, D. Xiao, J. Zhu, J. Zhang and X. x. Zhang, Adv. Electron. Mater, 2018, 4, 
1800205.

232. X. Lv, J. Wu, D. Xiao, H. Tao, Y. Yuan, J. Zhu, X. Wang and X. Lou, Dalton Trans., 2015, 44, 4440-4448.

233. X. Lv and J. Wu, J. Mater. Chem. C, 2019, 7, 2037-2048.

234. X. Lv, J. Wu, D. Xiao, J. Zhu and X. Zhang, J. Am. Ceram. Soc., 2018, 101, 1191-1200.

235. X. Lv, J. Wu, J. Zhu, D. Xiao and X. x. Zhang, J. Am. Ceram. Soc., 2018, 101, 4084-4094.

236. X. Lv, J. Wu, C. Zhao, D. Xiao, J. Zhu, Z. Zhang, C. Zhang and X.-x. Zhang, J. Eur. Ceram. Soc., 2019, 39, 305-315.

237. X. Lv, J. Wu, J. Zhu, D. Xiao and X. Zhangb, J. Eur. Ceram. Soc., 2017, 38, 85-94.

238. X. Lv, Z. Li, J. Wu, J. Xi, M. Gong, D. Xiao and J. Zhu, Mater. Des., 2016, 109, 609-614.

239. X. Lv, J. Wu, J. Zhu and D. Xiao, Phys. Chem. Chem. Phys., 2018, 20, 20149-20159.

240. X. Lv, J. Wu, D. Xiao, Y. Yuan, H. Tao, J. Zhu, X. Wang and X. Lou, RSC Adv., 2015, 5, 3929539302.

241. X. Cheng, J. Wu, X. Wang, B. Zhang, X. Lou, X. Wang, D. Xiao and J. Zhu, ACS Appl. Mater. Interfaces, 2013, 5, 10409-10417.

242. H. Tao, J. Wu, D. Xiao, J. Zhu, X. Wang and X. Lou, ACS Appl. Mater. Interfaces, 2014, 6, 20358-20364.

243. X. Wang, J. Wu, D. Xiao, X. Cheng, T. Zheng, X. Lou, B. Zhang and J. Zhu, ACS Appl. Mater. Interfaces, 2014, 6, 6177-6180.

244. B. Zhang, J. Wu, X. Cheng, X. Wang, D. Xiao, J. Zhu, X. Wang and X. Lou, ACS Appl. Mater. Interfaces, 2013, 5, 7718-7725.

245. T. Zheng, J. Wu, D. Xiao, J. Zhu, X. Wang and X. Lou, ACS Appl. Mater. Interfaces, 2015, 7, 
20332-20341.

246. T. Zheng, J. Wu, D. Xiao, J. Zhu, X. Wang, L. Xin and X. Lou, ACS Appl. Mater. Interfaces, 2015, 7, 5927-5937.

247. X. Cheng, J. Wu, X. Wang, B. Zhang, J. Zhu, D. Xiao, X. Wang and X. Lou, Appl. Phys. Lett., 2013, 103, 052906.

248. L. Jiang, Y. Li, J. Xing, J. Wu, Q. Chen, H. Liu, D. Xiao and J. Zhu, Ceram. Int., 2017, 43, 2100-2106.

249. C. Liu, D. Xiao, T. Huang, J. Wu, F. Li and J. Zhu, Ceram. Int., 2014, 40, 7589-7593.

250. Z. Tan, J. Zhu, Y. Zhang, J. Xing, Q. Chen, B. Wu, L. Sun, J. Wu, L. Jiang and D. Xiao, Ceram. Int., 2015, 41, 14610-14614.

251. C. Zhang, T. Zheng and J. Wu, Ceram. Int., 2016, 42, 16049-16054.

252. T. Zheng, J. Wu, X. Cheng, X. Wang, B. Zhang, D. Xiao, J. Zhu and X. Lou, Dalton Trans., 2014, 43, 9419-9426.

253. T. Zheng, J. Wu, X. Cheng, X. Wang, B. Zhang, D. Xiao, J. Zhu, X. Lou and X. Wang, Dalton Trans., 2014, 43, 11759-11766.

254. H. Tao, W. Wu and J. Wu, J. Alloys. Compd., 2016, 689, 759-766.

255. J. Wu, B. Zhang and W. Wu, J. Alloys. Compd., 2015, 651, 302-307.

256. R. Xiang and J. Wu, J. Alloys. Compd., 2016, 684, 397-402.

257. T. Zheng, Y. Zu and J. Wu, J. Alloys. Compd., 2015, 647, 927-934.

258. H. Tao, J. Wu, T. Zheng, X. Wang and X. Lou, J. Appl. Phys., 2015, 118, 044102.

259. J. Wu, X. Wang, X. Cheng, T. Zheng, B. Zhang, D. Xiao, J. Zhu and X. Lou, J. Appl. Phys., 2014, 115, 114104. 
260. J. Xing, Z. Tan, L. Jiang, Q. Chen, J. Wu, W. Zhang, D. Xiao and J. Zhu, J. Appl. Phys., 2016, 119, 034101.

261. X. Wang, J. Wu, D. Xiao, X. Cheng, T. Zheng, B. Zhang, X. Lou and J. Zhu, J. Mater. Chem. $A, 2014,2,4122-4126$.

262. C. Zhao, B. Wu, K. Wang, J.-F. Li, D. Xiao, J. Zhu and J. Wu, J. Mater. Chem. A, 2018, 6, $23736-23745$.

263. T. Zheng, J. Wu, D. Xiao, J. Zhu, X. Wang and X. Lou, J. Mater. Chem. A, 2015, 3, 1868-1874.

264. Q. Liu, Y. Zhang, L. Zhao, J. Gao, Z. Zhou, K. Wang, X. Zhang, L. Li and J.-F. Li, J. Mater. Chem. C, 2018, 6, 10618-10627.

265. T. Zheng, J. Wu, X. Cheng, X. Wang, B. Zhang, D. Xiao, J. Zhu, X. Wang and X. Lou, J. Mater. Chem. C, 2014, 2, 8796-8803.

266. J.-S. Zhou, K. Wang, F.-Z. Yao, T. Zheng, J. Wu, D. Xiao, J. Zhu and J.-F. Li, J. Mater. Chem. $C, 2015,3,8780-8787$.

267. L. Jiang, J. Xing, Z. Tan, J. Wu, Q. Chen, D. Xiao and J. Zhu, J. Mater. Sci., 2016, 51, 49634972.

268. F. Li, Q. Gou, J. Xing, Z. Tan, L. Jiang, L. Xie, J. Wu, W. Zhang, D. Xiao and J. Zhu, J. Mater. Sci: Mater. Electron., 2017, 28, 18090-18098.

269. F. Li, Z. Tan, J. Xing, L. Jiang, B. Wu, J. Wu, D. Xiao and J. Zhu, J. Mater. Sci: Mater. Electron., 2017, 28, 8803-8809.

270. D. Mazhao, D. Xiao, J. Wu and J. Zhu, J. Mater. Sci: Mater. Electron., 2015, 26, 7309-7315.

271. J. Wu, Y. Yang, X. Wang, D. Xiao and J. Zhu, J. Mater. Sci: Mater. Electron., 2014, 25, 46504656. 
272. X. Wang, J. Wu, X. Cheng, B. Zhang, D. Xiao, J. Zhu, X. Wang and X. Lou, J. Phys. D: Appl. Phys., 2013, 46, 495305.

273. X. Lv, J. Wu, J. Zhu, D. Xiao and X. Zhang, J. Eur. Ceram. Soc., 2018, 38, 85-94.

274. H. Tao and J. Wu, J. Eur. Ceram. Soc., 2016, 36, 1605-1612.

275. Z. Tan, J. Xing, L. Jiang, L. Sun, J. Wu, W. Zhang, D. Xiao and J. Zhu, RSC Adv., 2016, 6, 6983-6989.

276. Y. Qin, J. Zhang, W. Yao, C. Lu and S. Zhang, ACS Appl. Mater. Interfaces, 2016, 8, $7257-$ 7265.

277. W. Yang, P. Li, S. Wu, F. Li, B. Shen and J. Zhai, Adv. Electron. Mater, 2019, 5, 1900570.

278. M.-H. Zhang, K. Wang, J.-S. Zhou, J.-J. Zhou, X. Chu, X. Lv, J. Wu and J.-F. Li, Acta Mater, $2017, \mathbf{1 2 2}, 344-351$.

279. C. Shi, J. Ma, J. Wu, K. Chen and B. Wu, Ceram. Int., 2020, 46, 2798-2804.

280. W. Yang, P. Li, F. Li, X. Liu, B. Shen and J. Zhai, Ceram. Int., 2019, 45, 2275-2280.

281. Y. Yang, H. Wang, Y. Li, Q. Zheng, J. Liao, W. Jie and D. Lin, Dalton Trans., 2019, 48, 1067610682.

282. Y. Zhang, L. Li, B. Shen and J. Zhai, Dalton Trans., 2015, 44, 7797-7802.

283. D. Wang, F. Hussain, A. Khesro, A. Feteira, Y. Tian, Q. Zhao and I. M. Reaney, J. Am. Ceram. Soc., 2017, 100, 627-637.

284. J. Ma, J. Wu and B. Wu, RSC Adv., 2018, 8, 29871-29878.

285. H. Tao, C. Zhao, R. Zhang and J. Wu, J. Alloys. Compd., 2019, 795, 401-407.

286. K. Wang, F. Z. Yao, W. Jo, D. Gobeljic, V. V. Shvartsman, D. C. Lupascu, J. F. Li and J. Rödel, Adv. Funct. Mater., 2013, 23, 4079-4086. 
287. F.-Z. Yao, K. Wang, W. Jo, K. G. Webber, T. P. Comyn, J.-X. Ding, B. Xu, L.-Q. Cheng, M.-P. Zheng, Y.-D. Hou and J.-F. Li, Adv. Funct. Mater., 2016, 26, 1217-1224.

288. C. Shi, J. Ma, J. Wu, K. Chen and B. Wu, J. Mater. Sci: Mater. Electron., 2020, 31, 2809-2816.

289. W. Yang, P. Li, S. Wu, F. Li, B. Shen and J. Zhai, Ceram. Int., 2020, 46, 1390-1395.

290. C. Zhao, J. Yin, Y. Huang and J. Wu, Dalton Trans., 2019, 48, 11250-11258.

291. S.-T. Zhang, A. B. Kounga, E. Aulbach, W. Jo, T. Granzow, H. Ehrenberg and J. Rödel, J. Appl. Phys., 2008, 103, 034108-034101.

292. Q. Liu, J.-F. Li, L. Zhao, Y. Zhang, J. Gao, W. Sun, K. Wang and L. Li, J. Mater. Chem. C, $2018,6,1116-1125$.

293. J. Hao, Z. Xu, R. Chu, W. Li and J. Du, J. Mater. Sci: Mater. Electron., 2015, 26, 7867-7872.

294. Y. Huang, C. Zhao, B. Wu and J. Wu, J. Am. Ceram. Soc., 2019, 102, 2648-2657.

295. T. Leist, J. Chen, W. Jo, E. Aulbach, J. Suffner and J. Rödel, J. Am. Ceram. Soc., 2012, 95, 711 715.

296. R. Wang, K. Wang, F. Yao, J. F. Li, F. H. Schader, K. G. Webber, W. Jo and J. Rödel, J. Am. Ceram. Soc., 2015, 98, 2177-2182.

297. P. Li, B. Liu, B. Shen, J. Zhai, Y. Zhang, F. Li and X. Liu, J. Eur. Ceram. Soc., 2018, 38, 7583.

298. Y. Qin, J. Zhang, W. Yao, C. Wang and S. Zhang, J. Am. Ceram. Soc., 2015, 98, 1027-1033.

299. Y. Huan, X. Wang, Z. Shen, J. Kim, H. Zhou and L. Li, J. Am. Ceram. Soc., 2014, 97, 700-703.

300. T. Asada and Y. Koyama, Phys. Rev. B, 2007, 75, 214111.

301. X. Tan, H. He and J.-K. Shang, J. Mater. Res., 2005, 20, 1641-1653.

302. X. Tan, Z. Xu, J. K. Shang and P. Han, Appl. Phys. Lett., 2000, 77, 1529-1531. 
303. M. Hoffmann, M. Hammer, A. Endriss and D. Lupascu, Acta Mater., 2001, 49, 1301-1310.

304. J. Ricote, R. Whatmore and D. Barber, J. Phys.: Condens. Matter, 2000, 12, 323.

305. J. Gao, D. Xue, Y. Wang, D. Wang, L. Zhang, H. Wu, S. Guo, H. Bao, C. Zhou and W. Liu, Appl. Phys. Lett., 2011, 99, 092901.

306. S. Lu, Z. Xu, S. Su and R. Zuo, Appl. Phys. Lett., 2014, 105, 032903.

307. A. K. Singh, S. K. Mishra, Ragini, D. Pandey, S. Yoon, S. Baik and N. Shin, Appl. Phys. Lett., 2008, 92, 022910.

308. D. Damjanovic, Appl. Phys. Lett., 2010, 97, 062906.

309. L. Liu, M. Knapp, L. A. Schmitt, H. Ehrenberg, L. Fang, H. Fuess, M. Hoelzel and M. Hinterstein, EPL (Europhys. Lett.), 2016, 114, 47011.

310. Y. Huan, X. Wang, J. Koruza, K. Wang, K. G. Webber, Y. Hao and L. Li, Sci. Rep., 2016, 6, 22053.

311. C. Zhou, J. Zhang, W. Yao, D. Liu and W. Su, Scripta Mater., 2019, 162, 86-89.

312. M. Budimir, D. Damjanovic and N. Setter, J. Appl. Phys., 2003, 94, 6753-6761.

313. S.-E. Park and T. R. Shrout, J. Appl. Phys., 1997, 82, 1804-1811.

314. S.-E. Park, S. Wada, L. Cross and T. R. Shrout, J. Appl. Phys., 1999, 86, 2746-2750.

315. Y. Liu, G. Xu, J. Liu, D. Yang and X. Chen, J. Alloys. Compd., 2014, 603, 95-99.

316. L. Zheng, X. Huo, R. Wang, J. Wang, W. Jiang and W. Cao, CrystEngComm, 2013, 15, 77187722.

317. S. Gupta and S. Priya, Appl. Phys. Lett., 2011, 98, 242906.

318. L. Cross, Nature, 1958, 181, 178-179.

319. Y. Kizaki, Y. Noguchi and M. Miyayama, Appl. Phys. Lett., 2006, 89, 142910. 
320. K. Chen, G. Xu, D. Yang, X. Wang and J. Li, J. Appl. Phys., 2007, 101, 044103.

321. M. Davis, N. Klein, D. Damjanovic, N. Setter, A. Gross, V. Wesemann, S. Vernay and D. Rytz, Appl. Phys. Lett., 2007, 90, 062904.

322. J. G. Fisher, A. Benčan, J. Bernard, J. Holc, M. Kosec, S. Vernay and D. Rytz, J. Eur. Ceram. Soc., 2007, 27, 4103-4106.

323. J. G. Fisher, A. Benčan, J. Holc, M. Kosec, S. Vernay and D. Rytz, J. Cryst. Growth., 2007, 303, 487-492.

324. Y. Kizaki, Y. Noguchi and M. Miyayama, Key Eng. Mater., 2007, 350, 85-88.

325. J. G. Fisher, A. Benčan, J. Godnjavec and M. Kosec, J. Eur. Ceram. Soc., 2008, 28, 1657-1663.

326. J. G. Fisher, A. Benčan, M. Kosec, S. Vernay and D. Rytz, J. Am. Ceram. Soc., 2008, 91, $1503-$ 1507.

327. Y. Inagaki and K.-i. Kakimoto, Appl. Phys. Express., 2008, 1, 061602.

328. A. Benčan, E. Tchernychova, M. Godec, J. Fisher and M. Kosec, Microsc. Microanal., 2009, $15,435-440$.

329. H. Kimura, R. Tanahashi, K. Maiwa, H. Baba, Z. Cheng and X. Wang, Int. J. Mod. Phys. B, 2009, 23, 3631-3636.

330. D. Lin, Z. Li, Z. Xu and X. Yao, Ferroelectrics, 2009, 381, 1-8.

331. D. Lin, Z. Li, S. Zhang, Z. Xu and X. Yao, Solid. State. Commun., 2009, 149, 1646-1649.

332. Z. Zhou, J. Li, H. Tian, Z. Wang, Y. Li and R. Zhang, J. Phys. D: Appl. Phys., 2009, 42, 125405.

333. Y. Inagaki, K.-i. Kakimoto and I. Kagomiya, J. Eur. Ceram. Soc., 2010, 30, 301-306.

334. D. Lin, Z. Li, S. Zhang, M. Ma, Z. Xu and X. Yao, Ferroelectrics, 2010, 404, 200-206.

335. D. Lin, Z. Li, S. Zhang, Z. Xu and X. Yao, J. Am. Ceram. Soc., 2010, 93, 941-944. 
336. Y. Noguchi and M. Miyayama, J. Ceram. Soc. Jpn., 2010, 118, 711-716.

337. H. Uršič, A. Benčan, M. Škarabot, M. Godec and M. Kosec, J. Appl. Phys., 2010, 107, 033705.

338. H. Tian, C. Hu, Q. Chen and Z. Zhou, Mater. Lett., 2012, 68, 14-16.

339. R. Saravanan, D. Rajesh, S. Rajasekaran, R. Perumal, M. Chitra and R. Jayavel, Cryst. Res. Technol., 2013, 48, 22-28.

340. L.-Q. Cheng, K. Wang, J.-F. Li, Y. Liu and J. Li, J. Mater. Chem. C, 2014, 2, 9091-9098.

341. H. Deng, X. Zhao, H. Zhang, C. Chen, X. Li, D. Lin, B. Ren, J. Jiao and H. Luo, CrystEngComm, 2014, 16, 2760-2765.

342. X. Huo, L. Zheng, R. Zhang, R. Wang, J. Wang, S. Sang, Y. Wang, B. Yang and W. Cao, CrystEngComm, 2014, 16, 9828-9833.

343. X. Huo, L. Zheng, S. Zhang, R. Zhang, G. Liu, R. Wang, B. Yang, W. Cao and T. R. Shrout, Phys. Status Solidi RRL, 2014, 8, 86-90.

344. L. Zheng, S. Li, S. Sang, J. Wang, X. Huo, R. Wang, Z. Yuan and W. Cao, Appl. Phys. Lett., 2014, 105, 212902.

345. L. Zheng, J. Wang, X. Huo, R. Wang, S. Sang, S. Li, P. Zheng and W. Cao, J. Appl. Phys., 2014, 116, 044105 .

346. C.-W. Ahn, H.-Y. Lee, G. Han, S. Zhang, S.-Y. Choi, J.-J. Choi, J.-W. Kim, W.-H. Yoon, J.-H. Choi and D.-S. Park, Sci. Rep., 2015, 5, 17656.

347. X. Huo, R. Zhang, L. Zheng, S. Zhang, R. Wang, J. Wang, S. Sang, B. Yang and W. Cao, J. Am. Ceram. Soc., 2015, 98, 1829-1835.

348. M. Jiang, C. A. Randall, H. Guo, G. Rao, R. Tu, Z. Gu, G. Cheng, X. Liu, J. Zhang and Y. Li, J. Am. Ceram. Soc., 2015, 98, 2988-2996. 
349. D. Lin, S. Zhang, C. Cai and W. Liu, J. Appl. Phys., 2015, 117, 074103.

350. H. Tian, P. Tan, X. Meng, C. Hu, G. Shi, Z. Zhou and X. Wang, Cryst. Growth. Des., 2015, 16, $325-330$.

351. J. Wang, L. Zheng, B. Yang, R. Wang, X. Huo, S. Sang, J. Wu, Y. Chang, H. Ning and T. Lv, J. Cryst. Growth., 2015, 409, 39-43.

352. H. Liu, J. Koruza, P. Veber, D. Rytz, M. Maglione and J. Rödel, Appl. Phys. Lett., 2016, 109, 152902.

353. H. Liu, P. Veber, J. Koruza, D. Rytz, M. Josse, J. Rödel and M. Maglione, CrystEngComm, 2016, 18, 2081-2088.

354. J. Yang, F. Zhang, Q. Yang, Z. Liu, Y. Li, Y. Liu and Q. Zhang, Appl. Phys. Lett., 2016, 108, 182904.

355. C. Hu, H. Tian, X. Meng, G. Shi, W. Cao and Z. Zhou, RSC Adv., 2017, 7, 7003-7007.

356. H. Liu, P. Veber, A. Zintler, L. Molina-Luna, D. Rytz, M. Maglione and J. Koruza, IEEE Trans. Ultrason. Ferroelectr. Freq. Control, 2018, 65, 1508-1516.

357. H. Guo, C. Zhou, X. Ren and X. Tan, Phys. Rev. B, 2014, 89, 100104.

358. M. Zakhozheva, L. Schmitt, M. Acosta, W. Jo, J. Rödel and H.-J. Kleebe, Appl. Phys. Lett., 2014, 105, 112904.

359. M. Zakhozheva, L. Schmitt, M. Acosta, H. Guo, W. Jo, R. Schierholz, H.-J. Kleebe and X. Tan, Phys. Rev. Appl., 2015, 3, 064018.

360. H. Guo, B. K. Voas, S. Zhang, C. Zhou, X. Ren, S. P. Beckman and X. Tan, Phys. Rev. B, 2014, 90, 014103.

361. J. Fu and R. Zuo, Acta Mater., 2013, 61, 3687-3694. 
362. L. Jin, R. Huo, R. Guo, F. Li, D. Wang, Y. Tian, Q. Hu, X. Wei, Z. He and Y. Yan, ACS Appl. Mater. Interfaces, 2016, 8, 31109-31119.

363. C. Ang and Z. Yu, Adv. Mater., 2010, 18, 103-106.

364. K. Uchino, S. Nomura, L. E. Cross, R. E. Newnham and S. J. Jang, J. Mater. Sci., 1981, 16, $569-578$.

365. S.-Y. Choi, S.-J. Jeong, D.-S. Lee, M.-S. Kim, J.-S. Lee, J. H. Cho, B. I. Kim and Y. Ikuhara, Chem. Mater., 2012, 24, 3363-3369.

366. Y. Yang, Y. Ji, M. Fang, Z. Zhou, L. Zhang and X. Ren, Phys. Rev. Lett., 2019, 123, 137601.

367. M. C. Ehmke, F. H. Schader, K. G. Webber, J. Rödel, J. E. Blendell and K. J. Bowman, Acta Mater., 2014, 78, 37-45.

368. J. Gao, X. Hu, Y. Wang, Y. Liu, L. Zhang, X. Ke, L. Zhong, H. Zhao and X. Ren, Acta Mater. $2017, \mathbf{1 2 5}, 177-186$.

369. F. Cordero, F. Craciun, M. Dinescu, N. Scarisoreanu, C. Galassi, W. Schranz and V. Soprunyuk, Appl. Phys. Lett., 2014, 105, 232904.

370. J. Gao, X. Hu, L. Zhang, F. Li, L. Zhang, Y. Wang, Y. Hao, L. Zhong and X. Ren, Appl. Phys. Lett., 2014, 104, 252909.

371. D. S. Keeble, F. Benabdallah, P. A. Thomas, M. Maglione and J. Kreisel, Appl. Phys. Lett., 2013, 102, 092903.

372. D. Xue, Y. Zhou, H. Bao, J. Gao, C. Zhou and X. Ren, Appl. Phys. Lett., 2011, 99, 122901.

373. L. Zhang, M. Zhang, L. Wang, C. Zhou, Z. Zhang, Y. Yao, L. Zhang, D. Xue, X. Lou and X. Ren, Appl. Phys. Lett., 2014, 105, 162908.

374. C. Zhou, X. Ke, Y. Yao, S. Yang, Y. Ji, W. Liu, Y. Yang, L. Zhang, Y. Hao and S. Ren, Appl. 
Phys. Lett., 2018, 112, 182903.

375. C. Zhou, W. Liu, D. Xue, X. Ren, H. Bao, J. Gao and L. Zhang, Appl. Phys. Lett., 2012, 100, 222910.

376. M. Yin, C. Zhou, S. Ren, Y. Hao, M. Fang, W. Wang, J. Gao, T. Ma, L. Zhang and S. Yang, Ceram. Int., 2019, 45, 10304-10309.

377. C. Zhao, H. Wang, J. Xiong and J. Wu, Dalton Trans., 2016, 45, 6466-6480.

378. J. Gao, Y. Dai, X. Hu, X. Ke, L. Zhong, S. Li, L. Zhang, Y. Wang, D. Wang and Y. Wang, EPL (Europhys. Lett.), 2016, 115, 37001.

379. L. Zhao, X. Ke, W. Wang, M. Fang, A. Xiao, L. He, L. Zhang, J. Gao, Y. Wang and X. Ren, J. Alloys. Compd., 2019, 788, 748-755.

380. M. Abebe, K. Brajesh and R. Ranjan, J. Appl. Phys., 2017, 122, 034101.

381. J. Gao, L. Zhang, D. Xue, T. Kimoto, M. Song, L. Zhong and X. Ren, J. Appl. Phys., 2014, 115, 054108.

382. D. Xue, J. Gao, Y. Zhou, X. Ding, J. Sun, T. Lookman and X. Ren, J. Appl. Phys., 2015, 117, 124107.

383. D. Xue, Y. Zhou, H. Bao, C. Zhou, J. Gao and X. Ren, J. Appl. Phys., 2011, 109, 054110.

384. X. Hu, J. Gao, Y. Wang, Y. Liu, L. Li, D. Wang, F. Li, R. Yao, L. Zhong and X. Ren, J. Phys. Chem. C, 2019, 123, 15434-15440.

385. P. Thawong, C. Kornphom, S. Chootin and T. Bongkarn, Phase Transitions, 2016, 89, 232-241.

386. L. Zhang, X. Ren and M. A. Carpenter, Phys. Rev. B, 2017, 95, 054116.

387. P. Zheng, J. Zhang, Y. Tan and C. Wang, Acta Mater., 2012, 60, 5022-5030.

388. V. Hornebecq, C. Huber, M. Maglione, M. Antonietti and C. Elissalde, Adv. Funct. Mater., 2004, 
14, 899-904.

389. T. Karaki, K. Yan and M. Adachi, Appl. Phys. Express., 2008, 1, 111402.

390. L. Curecheriu, M. T. Buscaglia, V. Buscaglia, Z. Zhao and L. Mitoseriu, Appl. Phys. Lett., 2010, 97, 242909.

391. X. Deng, X. Wang, H. Wen, L. Chen, L. Chen and L. Li, Appl. Phys. Lett., 2006, 88, 252905.

392. X. Wang, X. Deng, H. Wen and L. Li, Appl. Phys. Lett., 2006, 89, 162902.

393. W. Luan, L. Gao, H. Kawaoka, T. Sekino and K. Niihara, Ceram. Int., 2004, 30, 405-410.

394. Y. Wu, J. Zhang, Y. Tan and P. Zheng, Ceram. Int., 2016, 42, 9815-9820.

395. M. Frey, Z. Xu, P. Han and D. Payne, Ferroelectrics, 1998, 206, 337-353.

396. T. Hoshina, Y. Kigoshi, S. Hatta, T. Teranishi, H. Takeda and T. Tsurumi, Ferroelectrics, 2010, 402, 29-36.

397. A. V. Polotai, A. V. Ragulya and C. A. Randall, Ferroelectrics, 2003, 288, 93-102.

398. D. Shihua, S. Tianxiu, Y. Xiaojing and L. Guanghua, Ferroelectrics, 2010, 402, 55-59.

399. T. Hoshina, T. Furuta, Y. Kigoshi, S. Hatta, N. Horiuchi, H. Takeda and T. Tsurumi, Jpn. J. Appl. Phys., 2010, 49, 09MC02.

400. T. Hoshina, T. Furuta, T. Yamazaki, H. Takeda and T. Tsurumi, Jpn. J. Appl. Phys., 2012, 51, 09LC04.

401. T. Hoshina, T. Furuta, T. Yamazaki, H. Takeda and T. Tsurumi, Jpn. J. Appl. Phys., 2013, 52, 09KC05.

402. T. Hoshina, S. Hatta, H. Takeda and T. Tsurumi, Jpn. J. Appl. Phys., 2018, 57, 0902BB.

403. T. Hoshina, Y. Kigoshi, S. Hatta, H. Takeda and T. Tsurumi, Jpn. J. Appl. Phys., 2009, 48, 09KC01. 
404. T. Hoshina, K. Takizawa, J. Li, T. Kasama, H. Kakemoto and T. Tsurumi, Jpn. J. Appl. Phys., 2008, 47, 7607.

405. T. Karaki, K. Yan and M. Adachi, Jpn. J. Appl. Phys., 2007, 46, 7035.

406. T. Karaki, K. Yan, T. Miyamoto and M. Adachi, Jpn. J. Appl. Phys., 2007, 46, L97.

407. L. Mitoseriu, D. Ricinschi, C. Harnagea, M. Okuyama, T. Tsukamoto and V. Tura, Jpn. J. Appl. Phys., 1996, 35, 5210.

408. P. Sapkota, S. Ueno, I. Fujii, G. P. Khanal, S. Kim and S. Wada, Jpn. J. Appl. Phys., 2019, 58, SLLC05.

409. H. Takahashi, Y. Numamoto, J. Tani, K. Matsuta, J. Qiu and S. Tsurekawa, Jpn. J. Appl. Phys., 2005, 45, L30.

410. H. Takahashi, Y. Numamoto, J. Tani and S. Tsurekawa, Jpn. J. Appl. Phys., 2007, 46, 7044.

411. H. Takahashi, Y. Numamoto, J. Tani and S. Tsurekawa, Jpn. J. Appl. Phys., 2008, 47, 8468.

412. V. R. Mudinepalli, L. Feng, W.-C. Lin and B. Murty, J. Adv. Ceram., 2015, 4, 46-53.

413. W. Cai, C. Fu, J. Gao and H. Chen, J. Alloys. Compd., 2009, 480, 870-873.

414. G. Arlt, D. Hennings and G. De With, J. Appl. Phys., 1985, 58, 1619-1625.

415. I. Fujii, M. Ugorek and S. Trolier-McKinstry, J. Appl. Phys., 2010, 107, 104116.

416. K. Kinoshita and A. Yamaji, J. Appl. Phys., 1976, 47, 371-373.

417. J. Qiu and Q. Jiang, J. Appl. Phys., 2009, 105, 034110.

418. B. Dai, X. Hu, R. Yin, W. Bai, F. Wen, J. Deng, L. Zheng, J. Du, P. Zheng and H. Qin, J. Mater. Sci: Mater. Electron., 2017, 28, 7928-7934.

419. F. Guo, B. Zhang, Z. Fan, X. Peng, Q. Yang, Y. Dong and R. Chen, J. Mater. Sci: Mater. Electron., 2016, 27, 5967-5971. 
420. Y. Shi, Y. Pu, Y. Cui and Y. Luo, J. Mater. Sci: Mater. Electron., 2017, 28, 13229-13235.

421. S. Shao, J. Zhang, Z. Zhang, P. Zheng, M. Zhao, J. Li and C. Wang, J. Phys. D: Appl. Phys., 2008, 41, 125408.

422. L. Curecheriu, S. B. Balmus, M. T. Buscaglia, V. Buscaglia, A. Ianculescu and L. Mitoseriu, J. Am. Ceram. Soc., 2012, 95, 3912-3921.

423. Y. Huan, X. Wang, J. Fang and L. Li, J. Am. Ceram. Soc., 2013, 96, 3369-3371.

424. B. Liu, X. Wang, R. Zhang and L. Li, J. Am. Ceram. Soc., 2017, 100, 3599-3607.

425. T. Hoshina, J. Ceram. Soc. Jpn., 2013, 121, 156-161.

426. G. P. Khanal, S. Kim, M. Kim, I. Fujii, S. Ueno and S. Wada, J. Ceram. Soc. Jpn., 2018, 126, 536-541.

427. Z.-Y. Shen and J.-F. Li, J. Ceram. Soc. Jpn., 2010, 118, 940-943.

428. B. Dai, P. Zheng, W. Bai, F. Wen, L. Li, W. Wu, Z. Ying and L. Zheng, J. Eur. Ceram. Soc., 2018, 38, 4212-4219.

429. Y. Huan, X. Wang, J. Fang and L. Li, J. Eur. Ceram. Soc., 2014, 34, 1445-1448.

430. C. Mao, S. Yan, S. Cao, C. Yao, F. Cao, G. Wang, X. Dong, X. Hu and C. Yang, J. Eur. Ceram. Soc., 2014, 34, 2933-2939.

431. Y. Tan, G. Viola, V. Koval, C. Yu, A. Mahajan, J. Zhang, H. Zhang, X. Zhou, N. V. Tarakina and H. Yan, J. Eur. Ceram. Soc., 2019, 39, 2064-2075.

432. C. Zhu, X. Wang, Q. Zhao, Z. Cai, Z. Cen and L. Li, J. Eur. Ceram. Soc., 2019, 39, 1142-1148.

433. B. Zhou, R. Li, J. Cai, J. Xu, Z. Zhao and J. Pei, Mater. Res. Express., 2018, 5, 095510.

434. C. Fang, L. Chen and D. Zhou, Phys. B, 2013, 409, 83-86.

435. Y. Tan, J. Zhang, C. Wang, G. Viola and H. Yan, Phys. Status Solidi A, 2015, 212, 433-438. 
436. H. Ghayour and M. Abdellahi, Powder Technol., 2016, 292, 84-93.

437. X. Li and J. Wang, Smart Mater. Struct., 2016, 26, 015013.

438. Z. Hanani, D. Mezzane, M. Amjoud, S. Fourcade, A. G. Razumnaya, I. A. Luk'Yanchuk and M. Gouné, Superlattices Microstruct., 2019, 127, 109-117.

439. T. Teranishi, S. Azuma and A. Kishimoto, Jpn. J. Appl. Phys., 2019, 58, SLLC03.

440. J. Scott, Science, 2007, 315, 954-959.

441. W. Buessem, L. Cross and A. Goswami, J. Am. Ceram. Soc., 1966, 49, 33-36.

442. H. Martirena and J. Burfoot, J. Phys. C: Solid State Phys., 1974, 7, 3182.

443. A. Bell, A. Moulson and L. Cross, Ferroelectrics, 1984, 54, 147-150.

444. G. Arlt, Ferroelectrics, 1987, 76, 451-458.

445. F. Bai, J. Li and D. Viehland, Appl. Phys. Lett., 2004, 85, 2313-2315.

446. H. Wang, J. Zhu, X. Zhang, Y. Tang and H. Luo, Appl. Phys. Lett., 2008, 92, 132906.

447. K. Kurushima, K. Kobayashi and S. Mori, IEEE Trans. Ultrason. Ferroelectr. Freq. Control, 2012, 59, 1900-1902.

448. F. Bai, J. Li and D. Viehland, J. Appl. Phys., 2005, 97, 054103.

449. J. Speck, M. De Graef, A. Wilkinson, A. Cheetham and D. Clarke, J. Appl. Phys., 1993, 73, 7261-7267.

450. S. Wada, K. Yako, K. Yokoo, H. Kakemoto and T. Tsurumi, Ferroelectrics, 2006, 334, 17-27.

451. D. Lin, S. Zhang, Z. Li, F. Li, Z. Xu, S. Wada, J. Luo and T. R. Shrout, J. Appl. Phys., 2011, 110, 084110.

452. X. Lou, J. Appl. Phys., 2009, 105, 024101.

453. J. Glaum and M. Hoffman, J. Am. Ceram. Soc., 2014, 97, 665-680. 
454. Z. Fan, J. Koruza, J. Rödel and X. Tan, Acta Mater., 2018, 151, 253-259.

455. Z. Fan, C. Zhou, X. Ren and X. Tan, Appl. Phys. Lett., 2017, 111, 252902.

456. Y. Zhang, J. Glaum, M. C. Ehmke, J. E. Blendell, K. J. Bowman and M. J. Hoffman, J. Am. Ceram. Soc., 2016, 99, 174-182.

457. Z. Fan and X. Tan, J. Eur. Ceram. Soc., 2018, 38, 3472-3477.

458. W. Jo, R. Dittmer, M. Acosta, J. Zang, C. Groh, E. Sapper, K. Wang and J. Rödel, J. Electroceram., 2012, 29, 71-93.

459. Z. Yang, B. Liu, L. Wei and Y. Hou, Mater. Res. Bull., 2008, 43, 81-89.

460. G. Smolensky, V. Isupov, A. Agranovskaya and N. Krainic, Fizika Tverdogo Tela, 1960, 2, 2982-2985.

461. G. Jones and P. Thomas, Acta Crystallogr. Sect. B: Struct. Sci., 2002, 58, 168-178.

462. I. Pronin, P. Syrnikov, V. Isupov, V. Egorov and N. Zaitseva, Ferroelectrics, 1980, 25, 395-397.

463. J. Zvirgzds, P. Kapostin, J. Zvirgzde and T. Kruzina, Ferroelectrics, 1982, 40, 75-77.

464. S. Vakhrushev, V. Isupov, B. Kvyatkovsky, N. Okuneva, I. Pronin, G. Smolensky and P. Syrnikov, Ferroelectrics, 1985, 63, 153-160.

465. C. W. Tai, S. H. Choy and H. L. Chan, J. Am. Ceram. Soc., 2008, 91, 3335-3341.

466. C. W. Tai and Y. Lereah, Appl. Phys. Lett., 2009, 95, 062901.

467. J. Zeng, K. Zhao, H. Zeng, L. Zheng, G. Li and Q. Yin, Mater. Lett., 2009, 63, 1468-1470.

468. L. Yunfei, L. Yinong and P. Zhigang, Ceram. Int., 2012, 38, 2679-2684.

469. I. Levin, I. Reaney, E. Anton, W. Jo, J. Rödel, J. Pokorny, L. Schmitt, H. Kleebe, M. Hinterstein and J. Jones, Phys. Rev. B, 2013, 87, 024113.

470. X. Chen, X. Xu, G. Yuan, M.-H. Lu, S.-T. Zhang, J. Yin and Z. Liu, Ceram. Int., 2014, 40, 
13961-13966.

471. A. Moosavi, M. Bahrevar, A. Aghaei, P. Ramos, M. Algueró and H. Amorín, J. Phys. D: Appl. Phys., 2014, 47, 055304.

472. J.-H. Cho, J.-S. Park, S.-W. Kim, Y.-H. Jeong, J.-S. Yun, W.-I. Park, Y.-W. Hong and J.-H. Paik, J. Eur. Ceram. Soc., 2017, 37, 3313-3318.

473. H. Muramatsu, H. Nagata and T. Takenaka, Jpn. J. Appl. Phys., 2016, 55, 10 TB07.

474. T. Miura, H. Nagata and T. Takenaka, Jpn. J. Appl. Phys., 2017, 56, 10PD05.

475. H. Nam, S. Kim, T. Aizawa, I. Fujii, S. Ueno and S. Wada, Ceram. Int., 2018, 44, S199-S202.

476. Z. T. Li, H. Liu, H. C. Thong, Z. Xu, M. H. Zhang, J. Yin, J. F. Li, K. Wang and J. Chen, $A d v$. Electron. Mater., 2019, 5, 1800756.

477. J. Zang, W. Jo and J. Rödel, Appl. Phys. Lett., 2013, 102, 032901.

478. J. Zhang, R.-X. Wang, L. Li, J.-Y. Wu, Y.-S. Cui, Z.-B. Gu, H. Zhang, M.-W. Zhu, S.-T. Zhang and B. Yang, J. Eur. Ceram. Soc., 2019, 39, 4705-4711.

479. K. Yoshii, Y. Hiruma, H. Nagata and T. Takenaka, Jpn. J. Appl. Phys., 2006, 45, 4493.

480. Y. R. Zhang, J. F. Li and B. P. Zhang, J. Am. Ceram. Soc., 2008, 91, 2716-2719.

481. Y.-R. Zhang, J.-F. Li, B.-P. Zhang and C.-E. Peng, J. Appl. Phys., 2008, 103, 074109.

482. A. Sasaki, T. Chiba, Y. Mamiya and E. Otsuki, Jpn. J. Appl. Phys., 1999, 38, 5564.

483. B.-H. Kim, S.-J. Han, J.-H. Kim, J.-H. Lee, B.-K. Ahn and Q. Xu, Ceram. Int., 2007, 33, 447452.

484. H. Lidjici, B. Lagoun, M. Berrahal, M. Rguitti, M. A. Hentatti and H. Khemakhem, J. Alloys. Compd., 2015, 618, 643-648.

485. B. Parija, T. Badapanda, S. Panigrahi and T. Sinha, J. Mater. Sci: Mater. Electron., 2013, 24, 
402-410.

486. C. Xu, D. Lin and K. Kwok, Solid State Sci., 2008, 10, 934-940.

487. J. Anthoniappen, C. H. Lin, C. Tu, P. Y. Chen, C. S. Chen, S. J. Chiu, H. Y. Lee, S. F. Wang and C. M. Hung, J. Am. Ceram. Soc., 2014, 97, 1890-1894.

488. H. Simons, J. Daniels, W. Jo, R. Dittmer, A. Studer, M. Avdeev, J. Rödel and M. Hoffman, Appl. Phys. Lett., 2011, 98, 082901.

489. B.-J. Chu, D.-R. Chen, G.-R. Li and Q.-R. Yin, J. Eur. Ceram. Soc., 2002, 22, 2115-2121.

490. T. Takenaka, K.-i. Maruyama and K. Sakata, Jpn. J. Appl. Phys., 1991, 30, 2236.

491. C. Ma, X. Tan, E. Dul'Kin and M. Roth, J. Appl. Phys., 2010, 108, 104105.

492. M. Otoničar, S. D. Škapin, B. Jančar, R. Ubic and D. Suvorov, J. Am. Ceram. Soc., 2010, 93, $4168-4173$.

493. C. Ma and X. Tan, J. Am. Ceram. Soc., 2011, 94, 4040-4044.

494. M. Otonicar, J. Park, M. Logar, G. Esteves, J. Jones and B. Jancar, Acta Mater, 2017, 127, 319 331.

495. X. Tan, C. Ma, J. Frederick, S. Beckman and K. G. Webber, J. Am. Ceram. Soc., 2011, 94, 4091-4107.

496. C. Ma, H. Guo, S. P. Beckman and X. Tan, Phys. Rev. Lett., 2012, 109, 107602.

497. J. E. Daniels, W. Jo, J. Rödel and J. L. Jones, Appl. Phys. Lett., 2009, 95, 032904.

498. Y. Guo, Y. Liu, R. L. Withers, F. Brink and H. Chen, Chem. Mater, 2010, 23, 219-228.

499. S. T. Zhang, A. B. Kounga, W. Jo, C. Jamin, K. Seifert, T. Granzow, J. Rödel and D. Damjanovic, Adv. Mater., 2009, 21, 4716-4720.

500. S.-T. Zhang, A. B. Kounga, E. Aulbach, H. Ehrenberg and J. Rödel, Appl. Phys. Lett., 2007, 91, 
112906.

501. S.-T. Zhang, A. B. Kounga, E. Aulbach, T. Granzow, W. Jo, H.-J. Kleebe and J. Rödel, J. Appl. Phys., 2008, 103, 034108.

502. S.-T. Zhang, A. B. Kounga, E. Aulbach, W. Jo, T. Granzow, H. Ehrenberg and J. Rödel, J. Appl. Phys., 2008, 103, 034107.

503. R. Dittmer, W. Jo, J. Rödel, S. Kalinin and N. Balke, Adv. Funct. Mater., 2012, 22, 4208-4215.

504. J. Kling, X. Tan, W. Jo, H. J. Kleebe, H. Fuess and J. Rödel, J. Am. Ceram. Soc., 2010, 93, 2452-2455.

505. J. Koruza, V. Rojas, L. Molina-Luna, U. Kunz, M. Duerrschnabel, H.-J. Kleebe and M. Acosta, J. Eur. Ceram. Soc., 2016, 36, 1009-1016.

506. Z. Fan and X. Tan, Appl. Phys. Lett., 2019, 114, 212901.

507. Z. Fan, L. Zhou, T.-H. Kim, J. Zhang, S.-T. Zhang and X. Tan, Phys. Rev. Mater., 2019, 3, 024402.

508. H. Guo, X. Liu, J. Rödel and X. Tan, Adv. Funct. Mater., 2015, 25, 270-277.

509. H. Simons, J. Glaum, J. E. Daniels, A. J. Studer, A. Liess, J. Rödel and M. Hoffman, J. Appl. Phys., 2012, 112, 044101.

510. F. Li, Q. Wang and H. Miao, J. Appl. Phys., 2017, 122, 074103.

511. F.-X. Li, D.-N. Fang and Y.-M. Liu, J. Appl. Phys., 2006, 100, 084101.

512. Q. Wang and F. Li, Sens. Actuators, A, 2018, 272, 212-216.

513. C. Qiu, B. Wang, N. Zhang, S. Zhang, J. Liu, D. Walker, Y. Wang, H. Tian, T. R. Shrout, Z. Xu, L-Q. Chen and F. Li, Nature, 2020, 577, 350-354.

514. Y. Wang, C. Luo, S. Wang, C. Chen, G. Yuan, H. Luo and D. Viehland, Adv. Electron. Mater., 
2020, 6, 1900949.

515. S. J. Derner and C. J. Kawamura, Apparatuses and methods including ferroelectric memory and for operating ferroelectric memory, U. S. Patent, 2018, 10074414B2.

516. W. A. Geideman, IEEE Trans. Ultrason. Ferroelectr. Freq. Control, 1991, 38, 704-711.

517. K. Kim and Y. J. Song, Microelectron. Reliab., 2003, 43, 385-398.

518. J. F. Scott, Ferroelectric memories, Springer Science \& Business Media, 2013.

519. J. Scott, Nat. Mater., 2007, 6, 256-257.

520. J. F. Scott and C. A. P. De Araujo, Science, 1989, 246, 1400-1405.

521. M. Wu, T. Zheng, H. Zheng, J. Li, W. Wang, M. Zhu, F. Li, G. Yue, Y. Gu and J. Wu, J. Mater. Chem. A, 2018, 6, 16439-16449.

522. L. Jiang, Y. Yang, R. Chen, G. Lu, R. Li, J. Xing, K. K. Shung, M. S. Humayun, J. Zhu and Y. Chen, Adv. Funct. Mater., 2019, 29, 1902522. 